\title{
SANDIA TECHNOLOGY
}

Engineering and Science Accomplishments

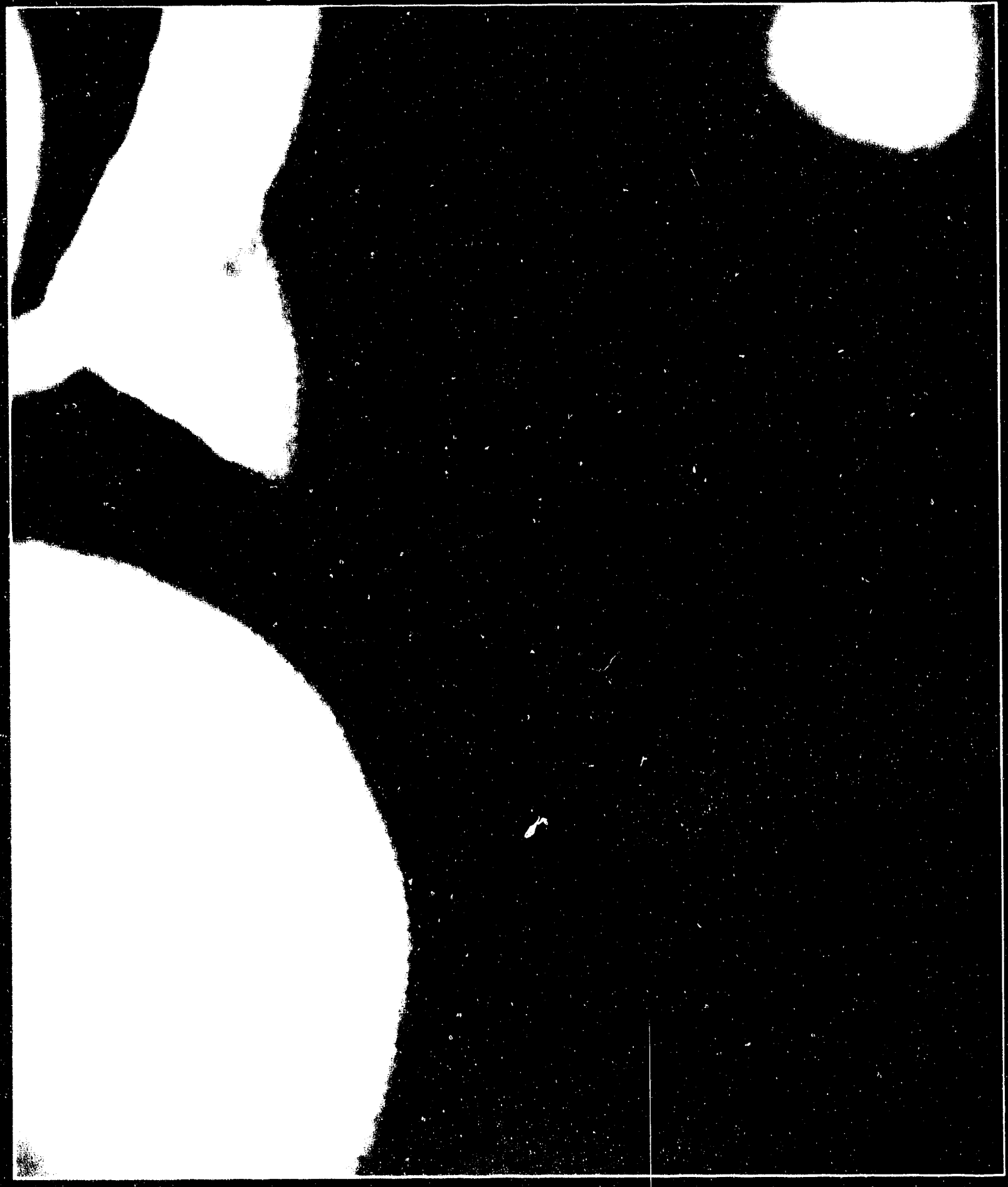

(17) Sandia National Laboratories

A Department of Energy multiprogram laboratory 


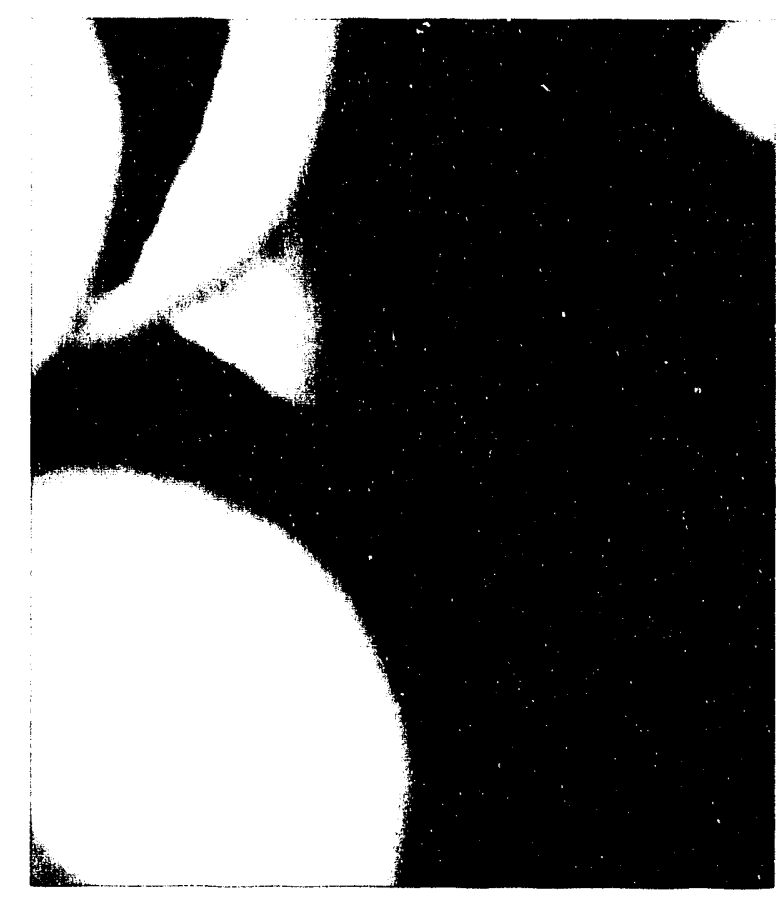

on the cover:

Molecular modeling at Sandia graphically represents the atoms in a molecule that might produce useful properties, such as catalysis. Here, a carbon dioxide molecule is held in the pocket of a synthetic metalloporphyrin molecule that mimics natural catalysts but can better survive harsh industrial environments.
Published by Sandia National Laboratories

Sandia is a multiprogram engineering and science laboratory operated by AT\&T for the Department of Energy with major facilities at Albuquerque, New Mexico, and Livermore, California, and a test range near Tonopah, Nevada. We have major research and development responsibilities for nuclear weapons, arms control, energy, the environment, economic competitiveness, and other areas of importance to the needs of the nation. Our principal mission is to support national defense policies by ensuring that the nuclear weapon stockpile meets the highest standards of safety, reliability, security, use control, and military performance. Selected technical activities and accomplishments are reported in two corporate publications: unclassified articles appear in Sandia Teclmology; classified work is reported in Sandia Weapon Reviere.

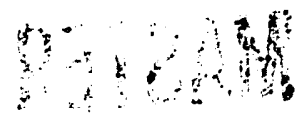




\section{SANDIA TECHNOLOGY \\ Engineering and Science Accomplishments}

1993

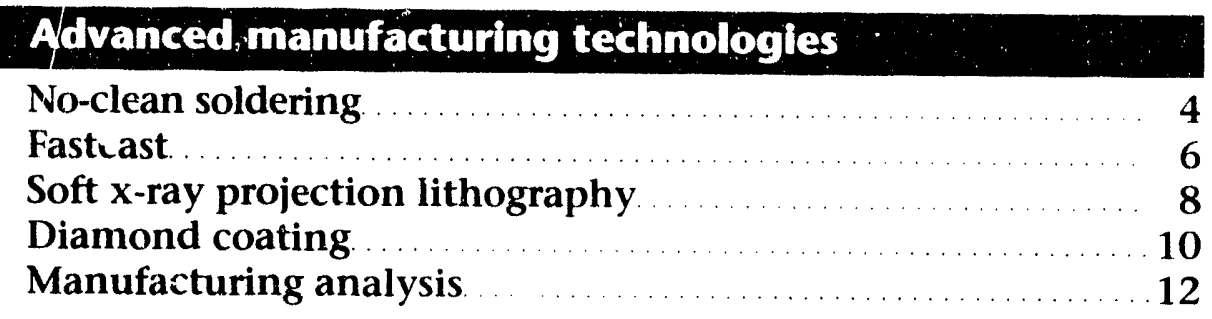

Intelligent machines.

Weapons dissassembly ............................... 16

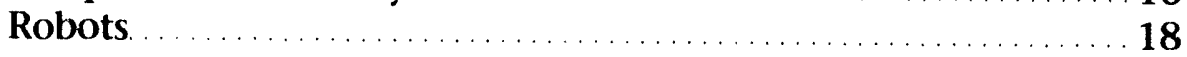

Computational simulation and high-performance computing

Super computing in medicine .......................... 22

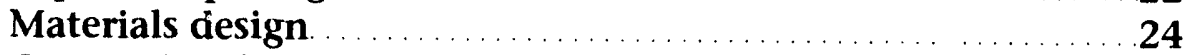

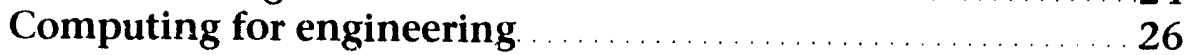

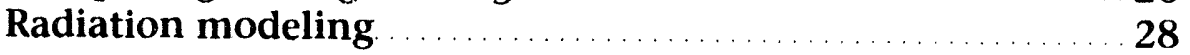

Sensors and instrumentation ...

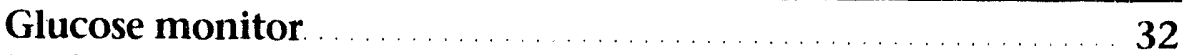

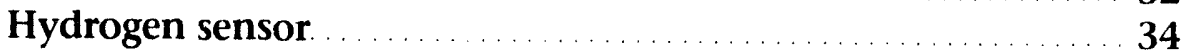

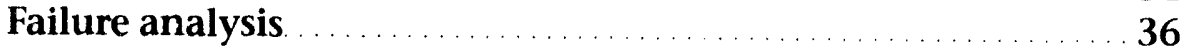

Climate studies ............................ 38

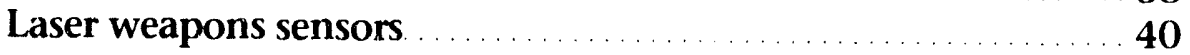

Information management

Human-machine interaction ...................... 44

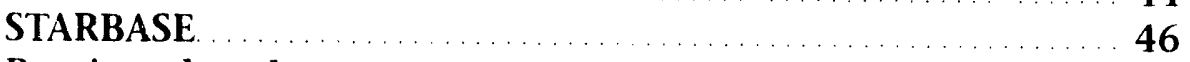

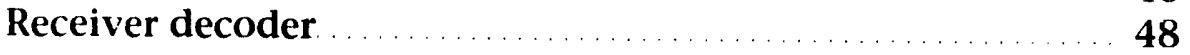

Energy and environment

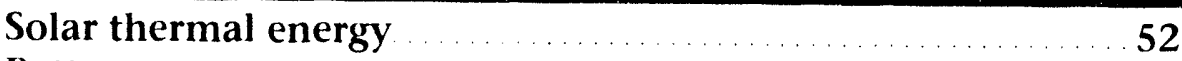

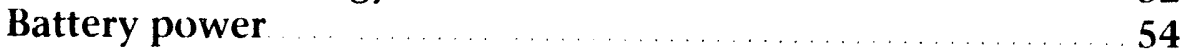

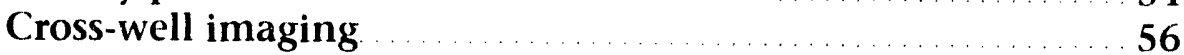

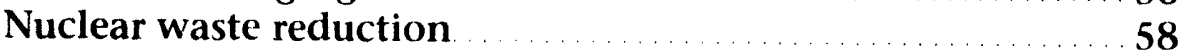

Weapons technology

Laser trigger ......................... 62

STARS guidance and control $\ldots \ldots \ldots \ldots \ldots \ldots \ldots$

\section{Awards and Patents}

Awards

Patents

68

70 


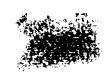

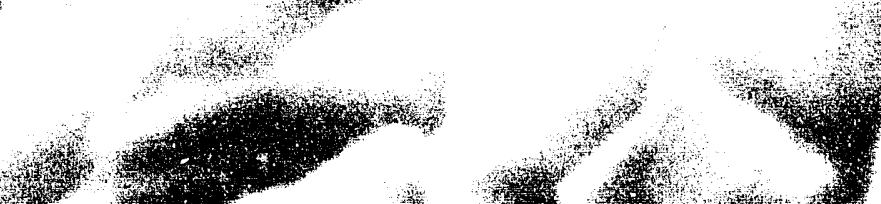

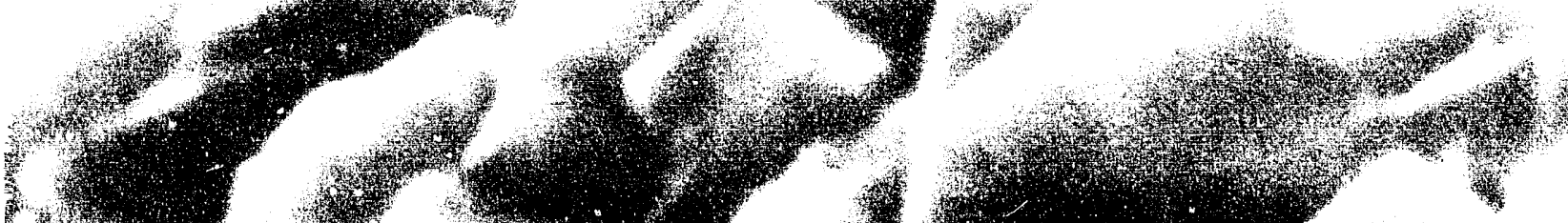
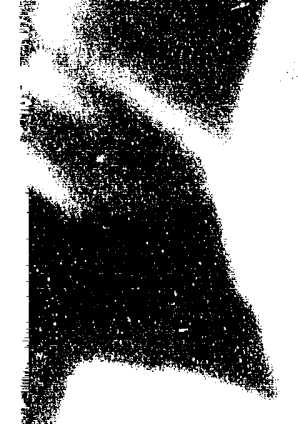

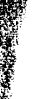

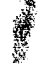

y

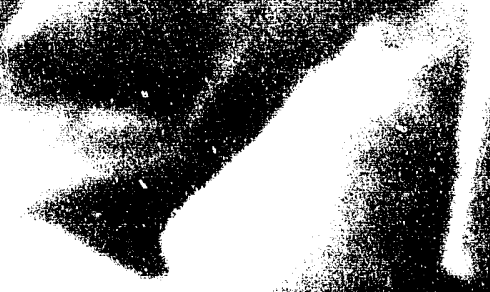

(1)

1x.

$4+4$
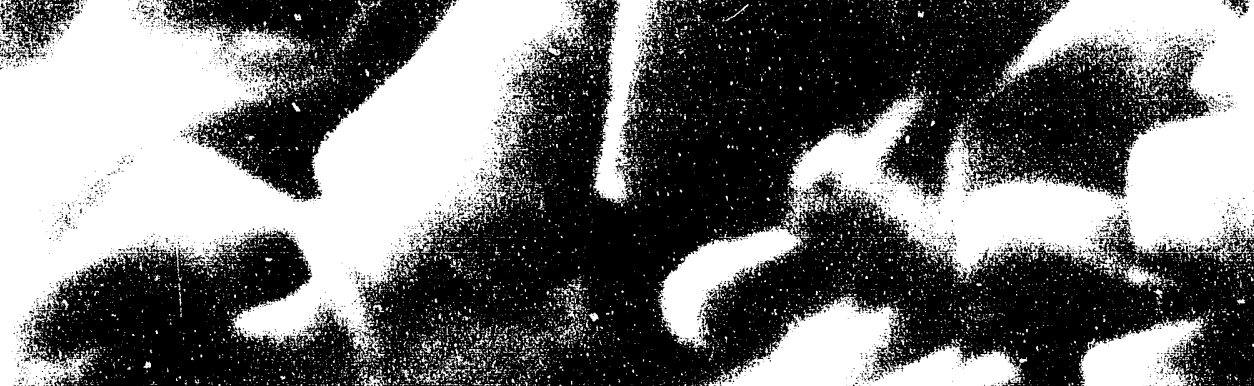

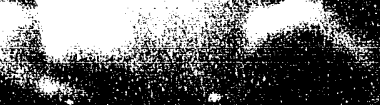

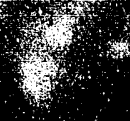

tis.
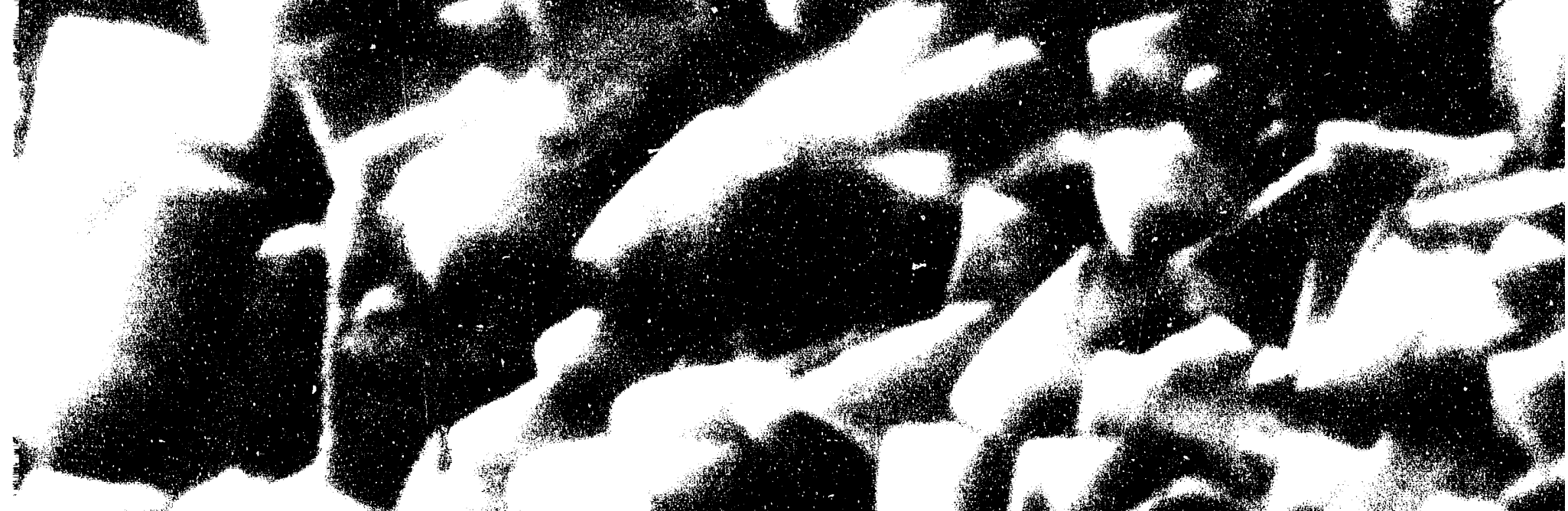

,

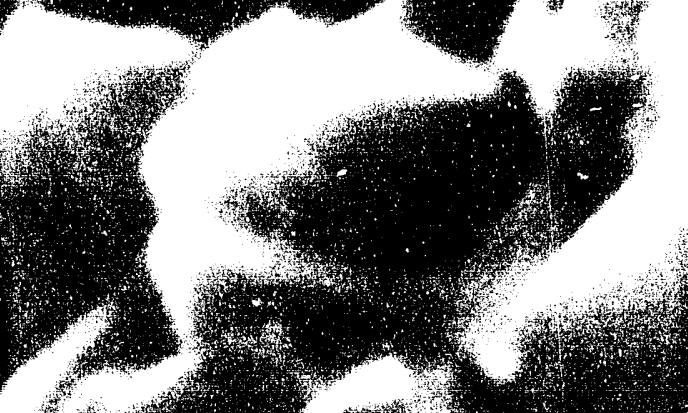

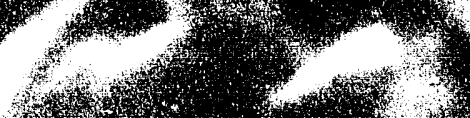

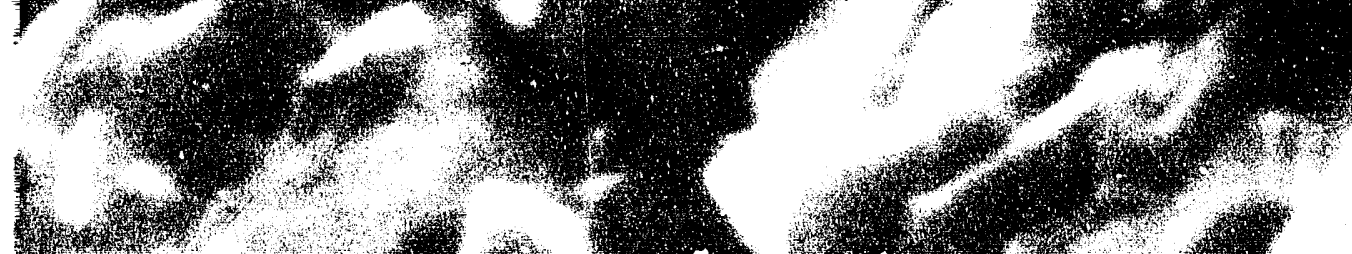

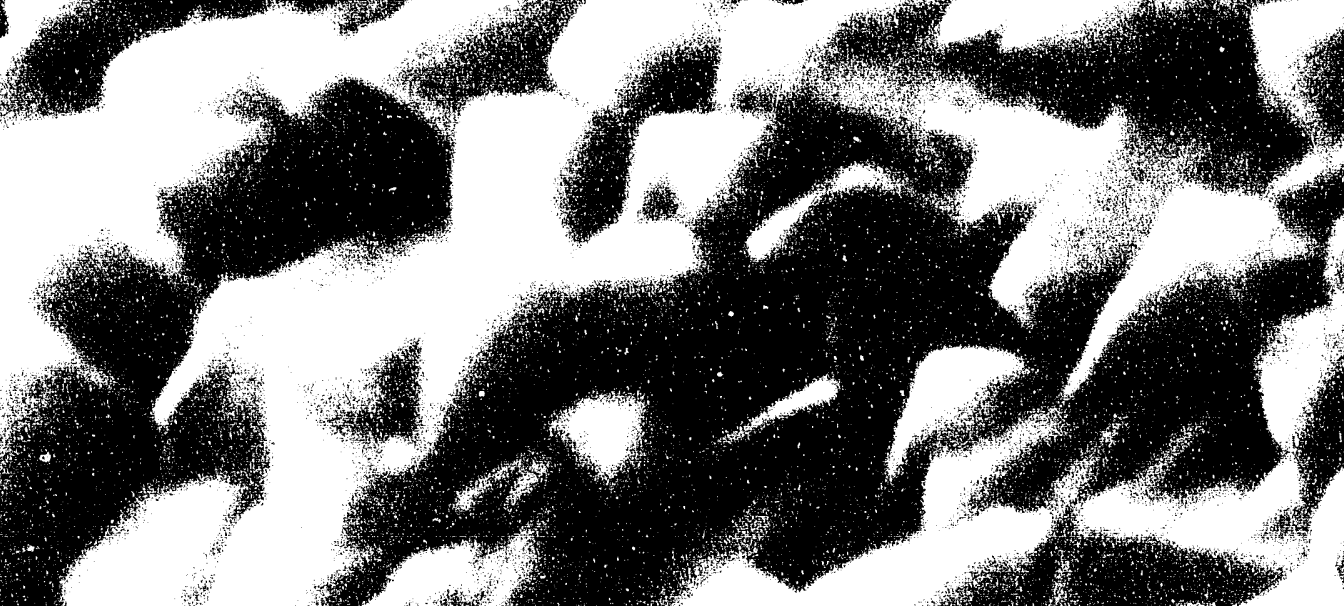
营
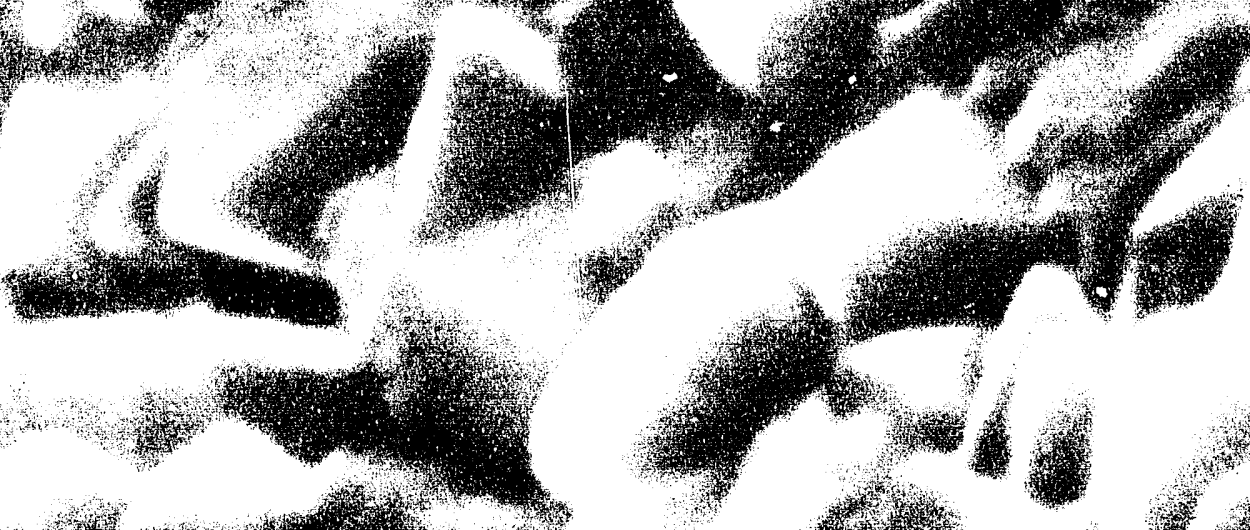

1.) $3 x$

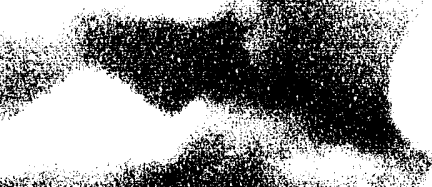

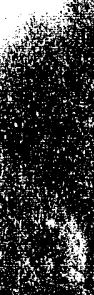

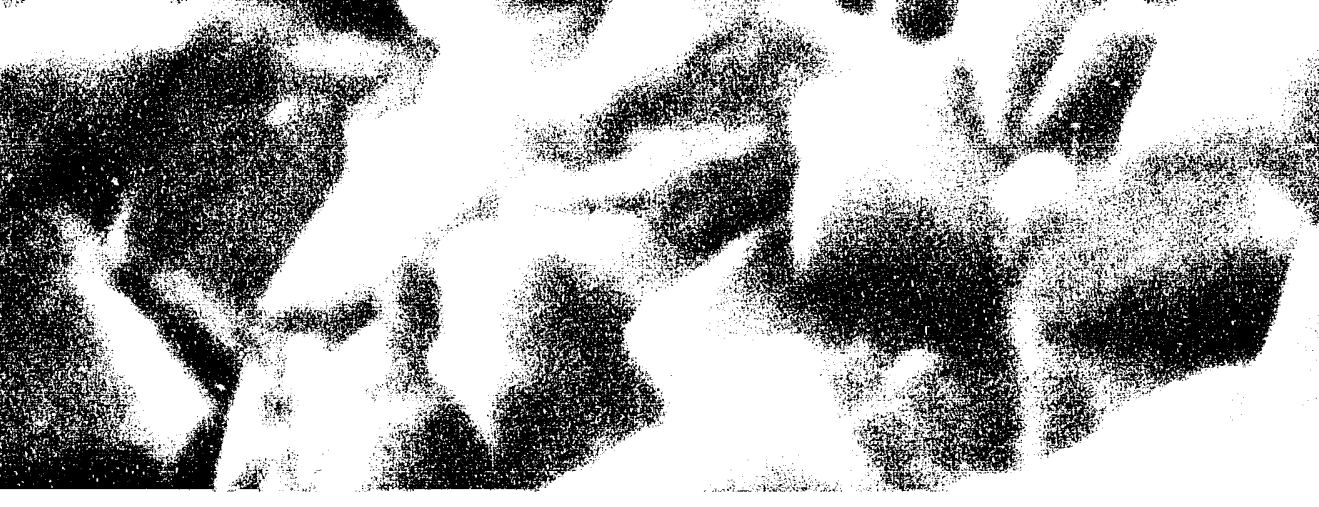

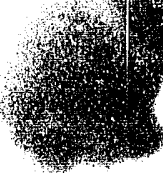


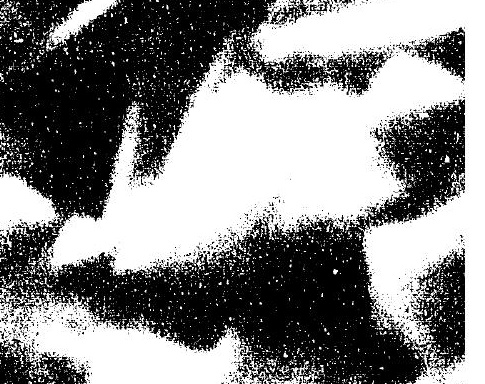


\section{No-clean soldering}

\section{Environmentally friendly soldering \\ process requires no solvents, destroys no ozone}

W ith the United States committed to phasing out ozone-depleting chemicals by 1995, American industry is getting a boost from Sandia and Motorola Inc., who have combined their talents to develop a new, solvent-free manufacturing process for printed circuit boards.

Dozens of companies have inquired about the no-clean soldering process, which is already having an impact on military specifications and has been demonstrated to be at least as reliable as traditional soldering methods.

Traditional printed circuit board manufacturing techniques use rosin flux to prepare a board for printed circuit application. Derived from pine trees, rosin is a wetting agent that helps solder stick to the surface of the board. Solder, a tin and lead alloy, is used to connect the components that power modern society's electronic tools, from computers to telephones to televisions. Although rosin itself is environmentally benign, after solder has been applied, solvents are needed to rinse the rosin residues from the board to prevent corrosion.

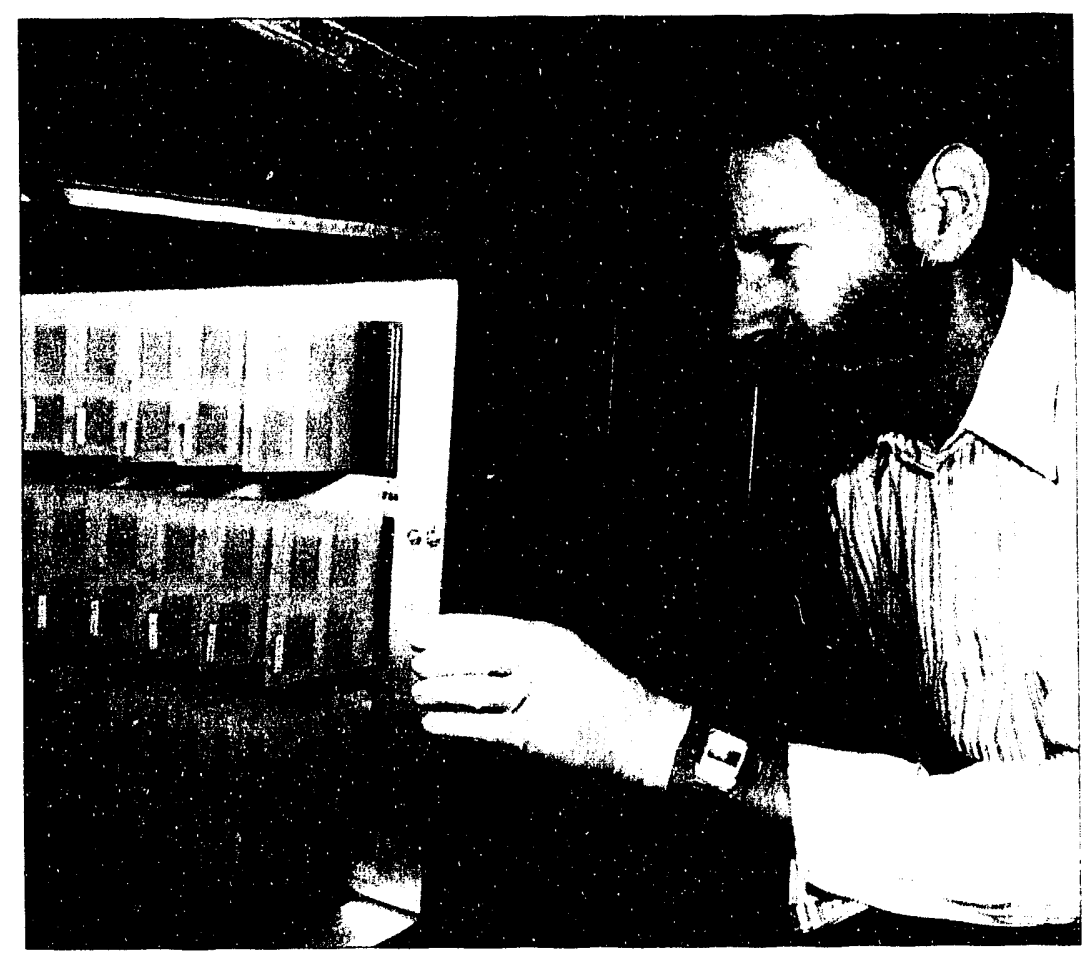

Solvent-based cleaning of electronic equipment accounts for at least one-fifth of the worldwide consumption of chlorofluorocarbons (CFCs), which deplete the ozone layer in the upper atmosphere and allow more harmful ultraviolet rays to reach the Earth's surface. High exposure to ultraviolet radiation has been linked to skin cancer and cataracts and may damage the body's immune system as well as interfere with plant photosynthesis and phytoplankton growth.

\section{An environmentally friendly process}

The new no-clean soldering process uses adipic acid, an additive employed by the food industry to give gelatin a tart flavor, as a wetting agent. In addition, the process uses formic acid, a substance found in ants and plants that breaks down into carbon dioxide and water. The process also uses nitrogen gas to eliminate oxygen inside the soldering chamber and inhibit oxidation. Processes such as these are an integral part of Sandia's Environmentally Conscious Manufacturing programs, which are dedicated to developing new technologies that are environmentally benign.

Unlike many programs to protect the environment, the new soldering process has the added distinction of being cheaper than conventional manufacturing processes. A manufacturer can save substantially by switching from rosin-flux, solventbased processes to the new process, estimates project manager Ronald Iman of Sandia's Manufacturing Systems Reliability Modeling Department.

"A typical 25-foot-long machine used by industry to clean printed circuit boards after soldering costs $\$ 100,000$ to $\$ 200,000$ a year for equipment,

Project manager Ron Iman inspects printed wiring boards that have undergone surface insulation resistance testing. 


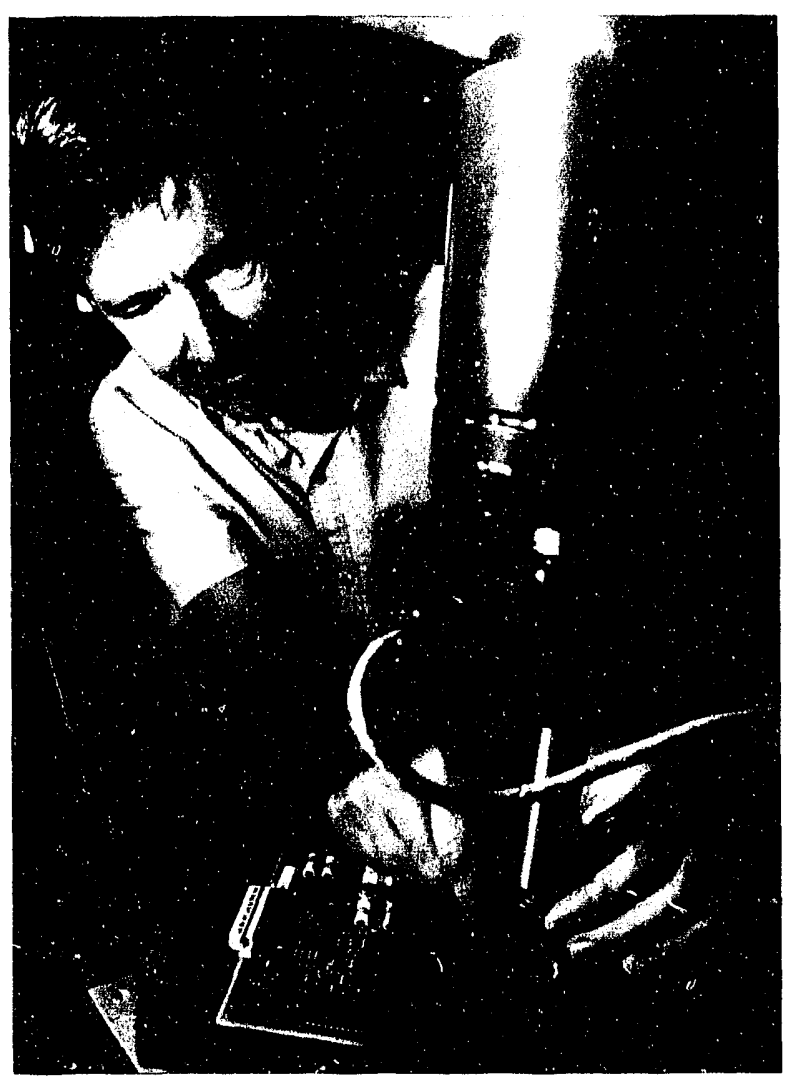

Sandia technician loe Kubas (above) tests the breaking strength of soldered joints in a printed circuit board manufactured without solvents. A close-up of the same test (below) shows a pulling chuck being lowered through a test frame to grasp a pin.

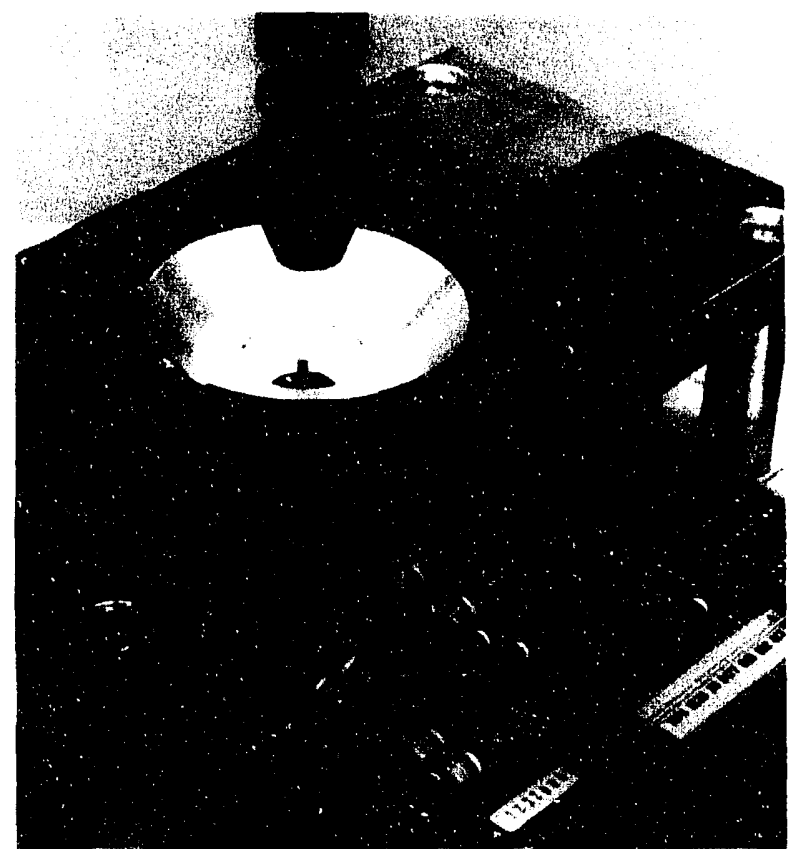

labor, maintenance, floor space, and electricity," says Iman. "Plus the machine adds time to the manufacturing process. The new process eliminates the need for the cleaning machine."

With funding from the l)epartment of linergy's Industrial Waste Reduction Program, Sandia, Los Alamos National Laboratory, and Motorola's Government Electronics Group in Scottsdale, Arizona, conducted tests to evaluate the performance and reliability of the process.

Initial results point overwhelmingly to its effectiveness. In tests rating electrical resistance, mechanical pull (the strength of the solder joints), surface insulation resistance at elevated temperatures and humidity, ionic cleanliness, visual defects, and long-term storage effects, the boards performed as well as or better than boards manufactured with conventional processes.

For instance, it took an average of 56 pounds of force to pull pins that had been soldered to the board, and an average of 65 pounds to remove resistors. Military specifications require a minimum strength of 4 pounds.

One of the goals of the cooperative venture is to change the military services' specifications for printed circuit boards, says Iman. Currently, those specifications require solvent cleaning, but the military is already granting exceptions on a case-by-case basis. Military standards are the benchmark for about 50 percent of all commercial electronic products as well, especially among smaller companies that cannot afford to conduct testing programs to demonstrate new processes or set their own standards.

If the military approves the no-clean technology, manufacturers will no longer need to conduct extensive testing programs to prove its reliability for each project that uses it. 团

For more information, call

Ron Iman, ()rg. 6613, (505) 844-88.34. 
Nen investment casting process

promises to save time,

redace costs of taking a part

from conception to creation

$\mathrm{N}$ ame just about any aircraft engine, and it's a sure bet that many of the components were manufactured by investment casting. A process ubiquitous throughout industry, investment casting is a means of creating complex metal objects by pouring molten steel into a mold and allowing it to cool. The process is especially effective for intricate, multidimensional objects such as golf club heads or weapon subsystem housings.

Investment casting is also referred to as the lost-wax process and has been used since ancient times to produce gold jewelry and other art objects. A wax replica of the part is made and then "invested" with ceramic by successive dipping into a ceramic slurry. Next, the wax is melted, leaving a ceramic cavity or mold, which is fired at high temperature. Then molten metal is poured into the mold through interconnected channels called gates. The gates allow the molten metal to flow evenly into the mold cavity.

Unfortunately for manufacturers, investment casting is also one of the most time-consuming aspects of getting a new product to market. To manufacture a new gas turbine, for example, a

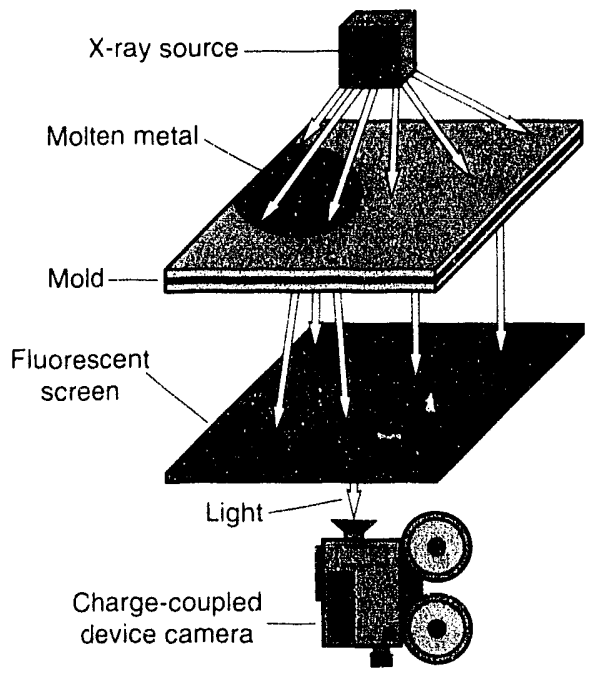

Diagram shows how $x$-rays are used to make images of molten metal as it fills a mold cavity. The $x$-rays illuminate a fluorescent screen that is photographed by a camera. company must first acquire tooling and then go through a development phase to create the wax part and the gating system. The entire process can take more than a year.

Through a program called Fastcast, Sandia is working with industry to speed up the casting process by building a casting tool kit that is basically a set of user-friendly computer algorithms that control product quality. The program combines Sandia's experience in high-performance computing and experimentation with the Department of Energy's efforts to share technologies developed at the national laboratories with private industry.

"The goal of Fastcast is to produce a set of 'rules' for the investment casting process that ensures a high-quality product without spending a lot of time developing it," says Frank Zanner, a scientist in Sandia's Liquid Metal Processing Department, who along with other researchers is helping to bring rinetal casting technology into the computer age.

Fastcast innovations include computer algorithms to characterize how metal flows into a mold, predict the physical characteristics of the finished product, and simulate the manufacturing process. In addition, the Fastcast program can eliminate the need for expensive tooling such as injection molds that are time-consuming to make. To produce a wax pattern, Sandia uses a state-of-the-art, selective laser sintering process that creates a solid pattern directly from a computer-generated, three-dimensional model.

The three-dimensional model can be a collaborative design created simultaneously on several different computers through a process known as concurrent engineering. Once the design is completed, a selective laser sintering process creates the wax pattern layer by layer, from bottom to top, from a bed of wax powder.

\section{The investment casting process}

"We have reduced the development time for a new casting from 50 weeks to 14 weeks, and we'd like to get it down to two weeks," says Frank. "We've already delivered relatively uncomplicated products, such as optical firing set housings for weapon systems, in one month."

Seven companies that produce investment castings have expressed interest in Fastcast, and Sandia is currently helping to put together a 
consortium of companies to share the cost of future development.

"Rapid prototyping processes such as stereolithography and selective laser sintering have caused a great deal of excitement in the manufacturing community because of the promise they hold for true 'art-to-part' production," says Sandia team leader Clint Atwood. "Investment casting will be a significant beneficiary of the ability to produce wax patterns directly from an electronic, feature-based solid model."

To better understand the process and improve gating designs, Sandia researchers are using an $x$-ray system that makes images of the molten metal as it is filling the mold. During these experiments, stainless steel heated to about $2900^{\circ} \mathrm{F}$ is poured into a mold. $X$-rays pass through the mold and illuminate a fluorescent screen. A video camera captures the image on the screen, providing views of the metalflowing through the gates to form the casting.

"This allows us to actually watch the metal filling the mold," says Zanner, "and develop the rules for casting that are part of the casting tool kit. These rules enable us to prevent metal shrinkage defects and minimize distortion before the casting is produced."

Sandia-designed computer codes that predict the physical properties of the casting are then compared with experimental results and updated as needed. The codes analyze thermal conditions, fluid flow, and stress. This process is guided by an automated computer algorithm that divides the solid model into a brick-type computational mesh. Ultimately, numerical simulations will provide all the information needed to eliminate gucsswork and design the optimum configuration for all the variables - gates, molds, temperatures - involved in a casting operation.

Future developments will make lastcast even more efficient and user-friendly. For example, one idea already being discussed at Sandia is an

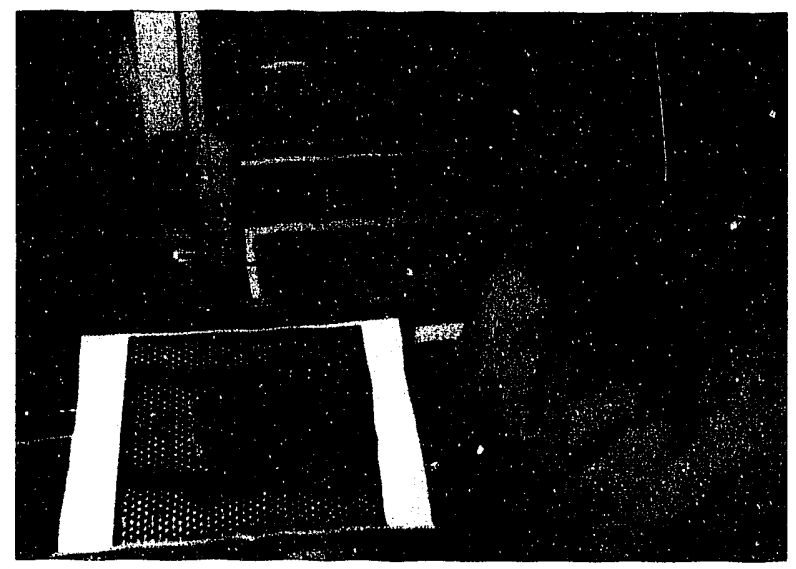

Gerry McCarty (above) holds a transfer tray with a platform on it that holds a part that has been built by a laser in a stereolithography machine. The part is a mockup of a component in a firing set assembly. Machinist Brian Pardo (below) moves a part heater inside a selective laser sintering machine to gain access to a finished wax pattern.

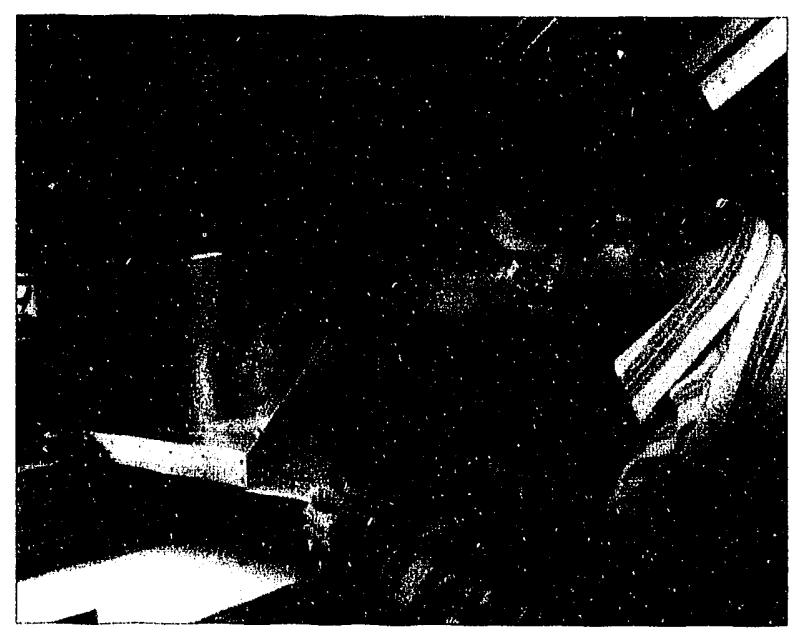

automated teller-style system that would give companies access to Sandia computers by keying in an identification number. This would allow them to participate in the concurrent engineering design process, for example, or enter data into analytical computer codes. 团

For more information, call

Frank Zanner, ()rg. 1833, (505) 845-3085, or

Clint Atwood (Rapid Prototyping), ()rg. 2484-1, (505) 844-()816, or Mike Maguire (Experimentation), Org. 18.31, (505) 845-3085, or Ron Sikorski (Architecture), (Org. 2814, (505) 844-5071, or David Gartling (Computation), ()rg. 1511, (505) 844.9150. 


\section{Soft x-ray projection lithography}

\section{High-tech project packs more microelectronics into smaller and smaller packages}

W hen it comes to microelectronics, the notion that good things come in small packages can be rewritten to say that better things come in smaller packages. This "shrinking" technology has fueled intense competition among microelectronics manufacturers to reduce the size of the features on integrated circuits (ICs).

Industry experts predict that eventually, feature sizes will become smaller than the wavelengths of light currently used to produce them. It is estimated that in less than 10 years, semiconductor devices will require advanced patterning technologies that can print lines less than 0.2 micrometers wide (a micrometer is approximately $1 / 100$ th the width of a human hair).

The challenge of producing such small features is somewhat like trying to paint a thin line with a wide brush. A promising technique, however, that may help produce devices on this scale is $\mathrm{x}$-ray projection lithography. Currently, researchers at Sandia and AT\&T Bell Laboratories are investigating this technique using soft $\mathrm{X}$-rays, under a Cooperative Research and Development Agreement. Soft $x$-rays have wavelengths from 50 to 250 angstroms (an angstrom is 10,000 times smaller than a micrometer) and provide finer resolution than does visible light. They also have lower energy than hard $x$-rays, the kind used in medical imaging, so they penetrate only a few tenths of micrometers into most materials. Hard $\mathrm{x}$-rays penetrate deeply, sometimes causing damage.

"Keeping pace with the competitive microelectronics manufacturing environment requires cooperation between national laboratories and US industry," says Sandia project manager Richard Stulen. "Sandia's continuing mission to provide research and development to enhance the nation's well-being will help US microelectronics manufacturers remain competitive in the world market."

\section{Reducing component size}

In projection lithography, a circuit diagram, or blueprint, is transferred from a mask to a silicon wafer. The process is similar to exposing a film negative onto photographic paper, except that the transferred image is reduced rather than enlarged to make the electronic components smaller. A camera composed of several mirrors images and reduces the circuit design. Sandia's current system has a reduction ratio of 20-to-1, that is, the image from a mask is reduced in size by a factor of 20 as it is printed onto a wafer. Because $x$-rays are much shorter than visible light rays, they can be used to achieve finerresolution images during the exposure process.

The use of shorter wavelengths does pose its own set of problems. Wavelengths shorter than about 1,800 angstroms are not transmitted through traditional optical-lens materials; instead, they are absorbed. Therefore, reduction systems must use reflective surfaces instead of glass lenses. But even with reflective optics, the $\mathrm{x}$-rays are strongly absorbed. To solve this problem, special multilayer coatings of synthetic materials have been developed that dramatically improve the reflection of soft $\mathrm{x}$-rays.

The ability to use reflection masks is an important feature of the process. The $x$-rays bounce off a highly reflective mirror that contains the IC pattern and focuses the reflected radiation onto the wafer.

Sandia has also been developing a laser-plasma source (LPS) for generating soft $x$-rays that is much less expensive than a synchrotron. While synchrotrons can cost more than $\$ 30$ million,

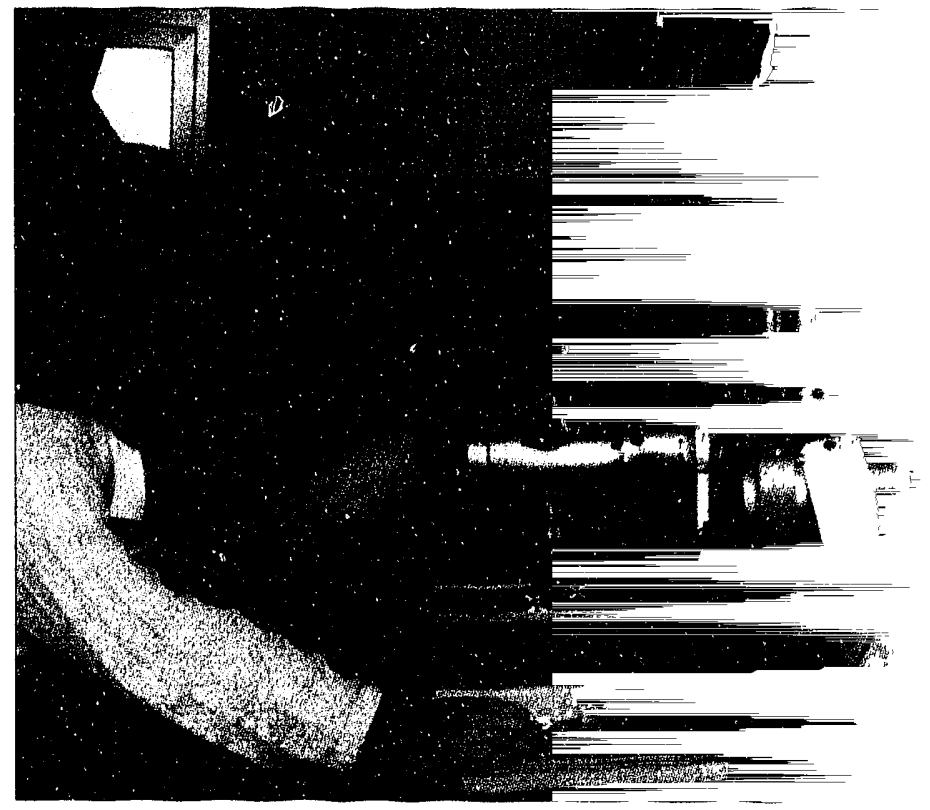


Sandia's LPS costs only about $\$ 500,(0) 0$. (Other sources of sott $x$-rays provide output too weak to support photolithography research.

The L.PS uses a krypton-fluoride ultraviolet laser that focuses 30-nanosecond pulses of light onto a gold target. When the laser pulse strikes the target, a small amount of material vaporizes from the surface. The remainder of the pulse heats the vaporized material further, raising it to higher temperatures. The vaporized material becomes an extremely hot plasma that produces a flash of radiation that includes soft $x$-rays.

Sandia is now developing a laboratory soft $x$-ray projection lithography system with AT\&T. Future manufacturing systems will likely require modifications for high-volume production of ICs. For example, the laser will probably have a high repetition rate and an average power level of about 500 watts. It will operate much like a strobe light illuminating a moving target. The mask, which will be a high-reflectivity mirror, will be more rugged than the fragile masks used in earlier experiments. 团

Fol mol' information, call

Richard H. Stulen, ()rg. 8.342, (5.10) 294-2070.

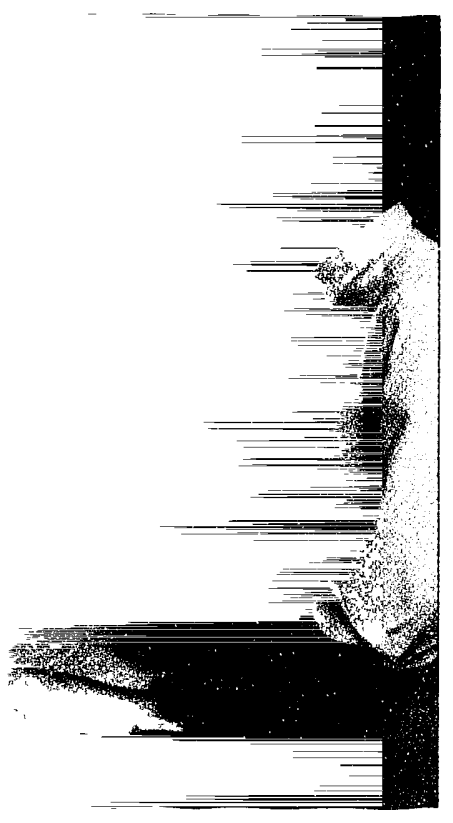

Researchers (from left) Kurt Berger, Steve Haney, and Randy Schmitt adjust a laser plasma source in the soft $x$-ray lithography facility.
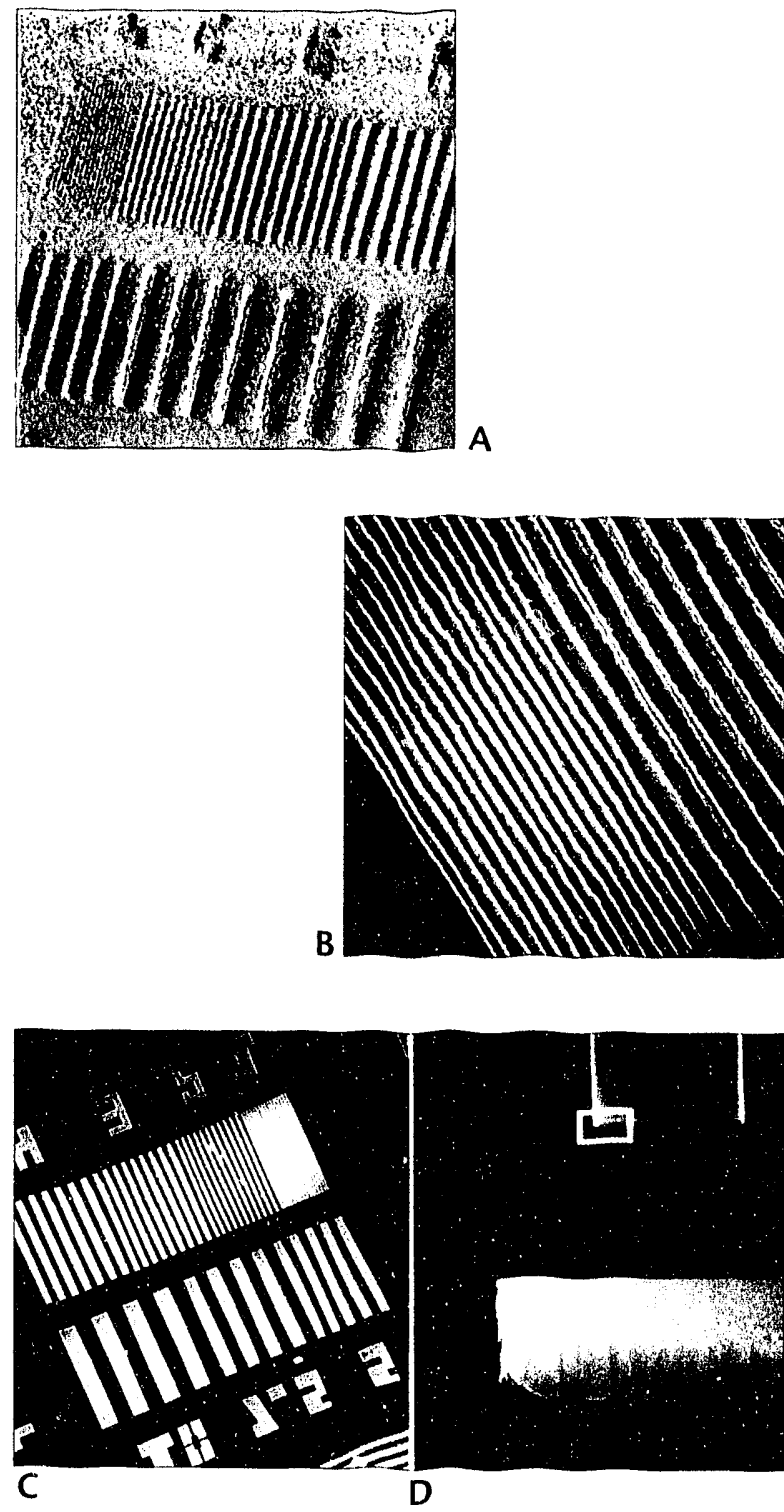

Early tests of x-ray projection lithography used a Schwarzschild camera to transfer a circuit diagram from a mask to a silicon wafer. The first tests using a laser plasma source at Sandia produced circuit features separated by 0.05 micrometer, as shown in an atomic force microscope image $(A)$. Similar results were obtained by AT\&T using synchrotron radiation (B). These features were produced using a mask whose smallest lines and spaces were separated by 1 micrometer, for a total image reduction of 20 to 1 . A reflecting mask made by coating a substrate with a layered synthetic material (LSM) and etching some of it away (C) shows bright and dark areas. Bright regions are reflective, dark are not. An enlargement of one of the reflecting lines (bright areas) shows individual layers oi inolybdenum and silicon that make up the LSM (D). 


\section{Diamond coatings}

\section{The latrilest substance can be manufactured with chemical vapors}

T echnology has come a long way since the days when it was believed that only nature could produce the pressures and temperatures necessary to form a diamond.

Today, scientists can produce a diamond film entirely in the laboratory, using only methane gas and an energy source such as a laser or microwave beam. The resulting.product is not a cheap imitation, but the real thing. It has the same atomic structure as the gemstone mined from deep within the earth, and it is created without trying to squeeze carbon atoms at enormous pressures of about 1 million psi.

This is good news to industries such as $\mathrm{Du}$ Pont, which recently signed a Cooperative Research and Developn.ant Agreement (CRADA) with Sandia and Los Alamos national labs to develop the capability to coat a variety of composite materials with diamond and diamond-like carbons. The cost of the three-year, \$6-million program will be shared equally by Du Pont and the Department of Energy. It is only one example of DOE's ongoing efforts to transfer technologies from weapon development to American industry.

Du Pont announced last fall that the collaborative effort is expected to generate a new class of high-strength composite materials with improved thermal conductivity. Besides being the hardest known substance on earth, making it the strongest by weight, diamond is an excellent thermal conductor, better than gold, silver, or copper (diamond has five times the thermal conductivity of copper, for example, at room temperature).

In fact, according to Sandia researcher Wen $\mathrm{Hsu}$, who has studied diamond films for several years, the high thermal conductivity of diamonds could be used to spread heat generated by densely packed electronic chips inside a computer. "This technology is a big step toward making a densely packed high-speed supercomputer," he says.

The formation of diamond films is an effort that has involved many Sandians over the years as part of the ongoing quest to create high-performance materials for both military and commercial applications. The weight reduction that is possible by using thin films of diamond make it an ideal substance for imparting strength to aircraft, spacecraft, automobiles, and construction materials. The films can be used as coatings on radiators or as substrates in electronic components, not to mention many other uses ranging from abrasion-resistant coatings to industrial grinding and polishing agents.

\section{Chemical vapor deposition}

The new process manufactures diamond-film coatings by chemical vapor deposition (CVD). The technology begins with methane gas, which reacts in the presence of microwaves and breaks down into hydrogen and carbon gases. The gases migrate to a cooler surface upon which the carbon condenses, forming polycrystalline diamond films. This process takes place inside a reaction chamber that uses intense heat or plasma to break down the hydrocarbon (in this case methane) and to dissociate molecular hydrogen. Such reactions typically take place at temperatures of about 4,000 degrees F.

During the past four years, Hsu and collaborator Dave Tung have been engaged in research aimed at understanding diamond growth techniques. To this end, they constructed two different kinds of CVD reactors - a hot-filament reactor and a microwave plasma reactor. The two reactors are similar to those used by commercial diamond manufacturers. In the hot-filament reactor, methane and hydrogen pass over a glowing tungsten filament to be broken down. In a microwave reactor, a microwave-heated plasma provides the energy to break down the methane and hydrogen. One of their experiments involved installing a mass spectrometer in each reactor to analyze the gas and surface chemistry in the deposition region.

Another series of experiments conducted by their colleagues, Michelle Schulberg and Glenn Kubiak, studied the kinetics of specific reactions on the surface of natural diamonds. Such experiments measured, for example, the rates of release of hydrogen and hydrocarbons from the surface of natural diamonds as a function of temperature.

The results of this research have been incorporated into a sophisticated numerical code that simulates the manufacturing environment and predicts the characteristics of the resulting diamond film. "This research has produced a better understanding of how diamonds are grown," says $\mathrm{Hsu}$, "and in the future, the information will be used as a predictive tool." 
Another element of Sandia's ongoing research is the development of reliable process sensors. To achieve optimum growth of diamond films, both the cooler surface upon which the diamond film is deposited and the gases used to form the diamond film must be continuously monitored.

Sandia has experimented with a variety of inproaches for monitoring film quality and temperature, using thermocouples, optical pyrometers, and laser spectroscopy.

"One of the goals of Sandia's research is to learn to form single-crystal diamond film at the lowest possible deposition temperature," says Hsu." At one time, we achieved $800 \mathrm{~K}$, and that was a record, but others reduced the temperature further to only $500 \mathrm{~K}$. We want to be able to diamond-coat plastic substrates." 团

For more information, call

Wen Hsu, Org. 8347, (510) 294-2379.

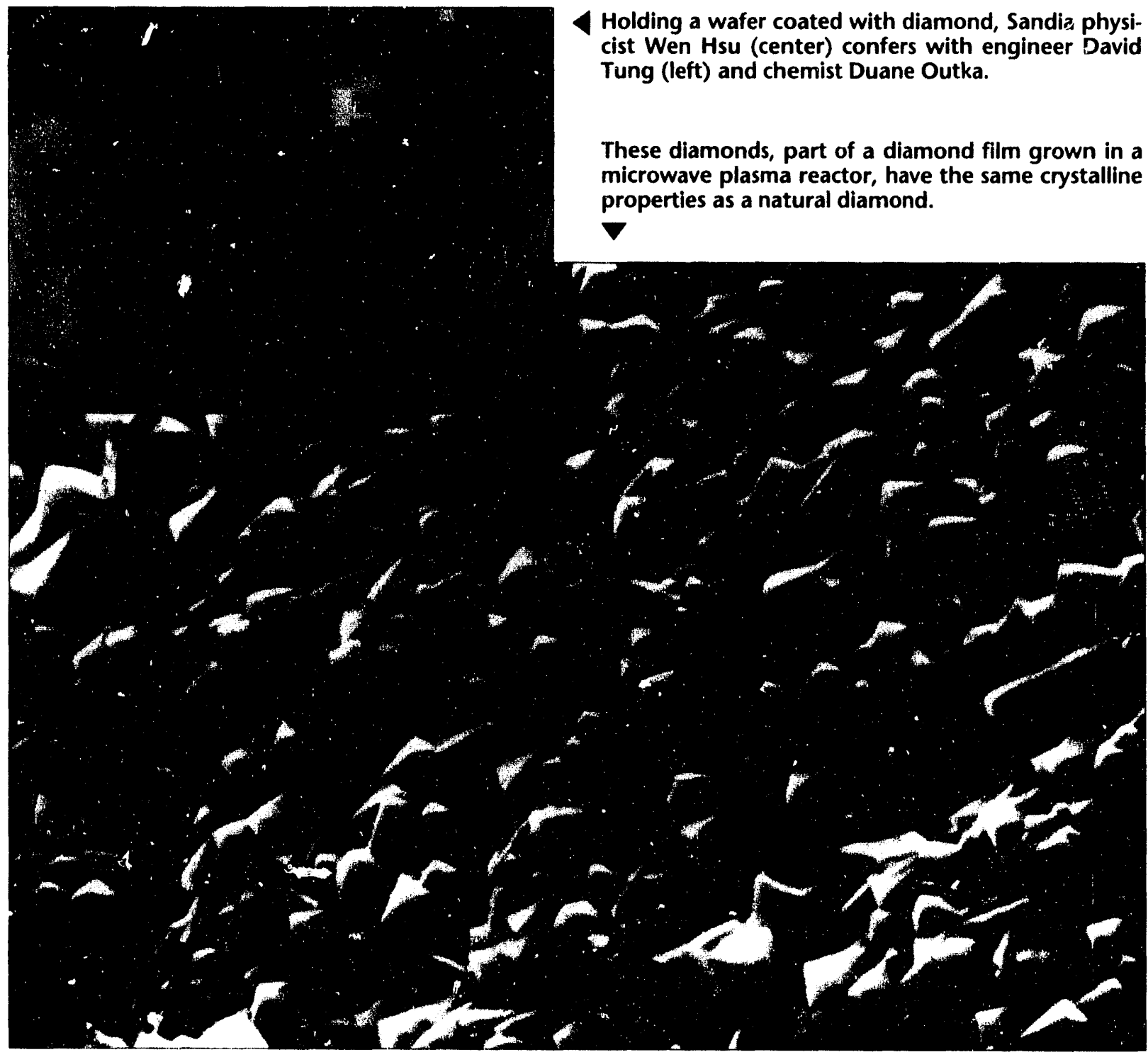




\section{Manufacturing analysis}

Computer modeling of fluid dynamics, structural mechanics improves manufacturing process

$\mathrm{A}$ n old Russian proverb reads, "Measure nine times, then cut." Even with such precautions, the irial-and-error method of making something is costly and time-consuming. To remain competitive in today's markets, American industry is turning to computer modeling to handle processes that have typically required

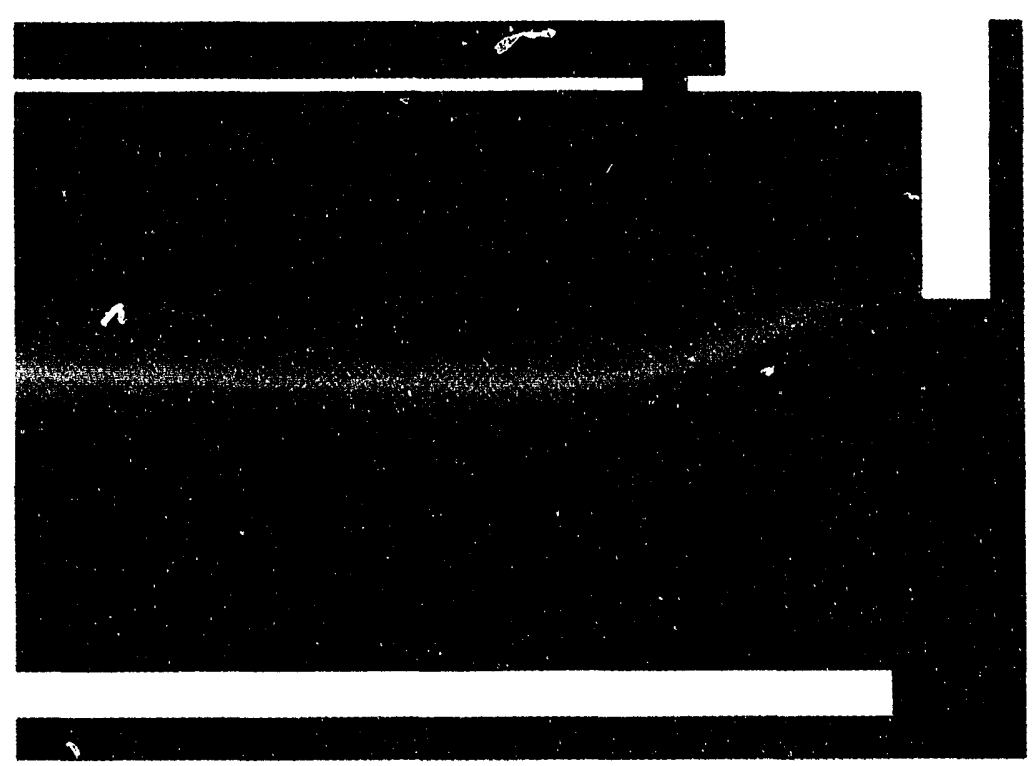

repeated modifications. Sandia is helping industry by developing numerical modeling for such manufacturing processes as semiconductor development, glassmaking, and welding and metal fabrication.

Many manufacturing processes create stresses and strains that can weaken or deform a finished part. Microscopic cracks, shrinkage, and residual stresses left inside a material after processing can reduce a part's lifetime or performance.

For example, at room temperature, glass is brittle and easily broken. To produce durable glass products, care must be taken to ensure that stresses generated during processing remain low. Sandia has developed a computer model that calculates stresses that occur as molten glass cools and hardens. Guided by these numerical predictions, manufacturing processes can be optimized. Analyses of a glass-tometal seal, typical of those found in electronic systems designed at Sandia, have been used to explain cracking and to identify changes in the cooling process that will mitigate fracture.

Similar problems exist during welding. Sandia researchers are working closely with industry to

By modeling a chemical vapor deposition (CVD) reactor, Sandia researchers were able to optimize the reactor's productivity by changing the reactor baffle model. This created a more uniform particle deposition (top) than the original baffle system (right).

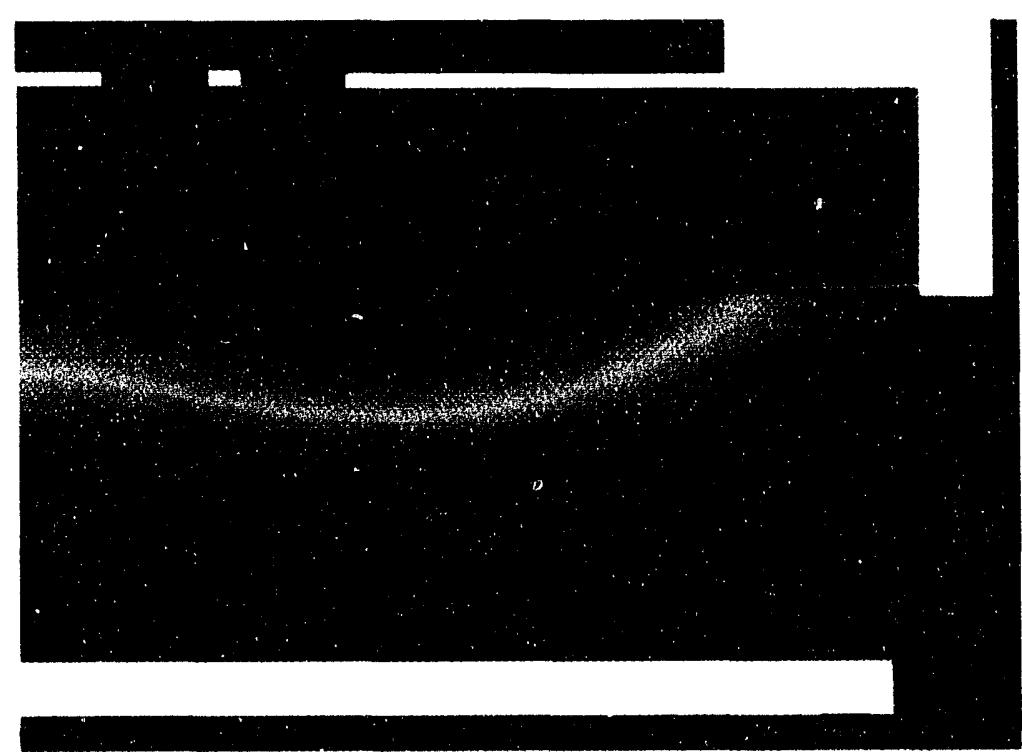


improve the quality of welded parts for such applications as aircraft engines. New computer models and improved measurement techniques have significantly increased understanding of the welding process. Sandia is developing sensors to measure temperature and distortion during welding. The resulting experimental data are used to generate input for computer simulations of welding processes. Sandia's computer modeling uses accurate mathematical descriptions of welded materials to predict the stresses and deformations that occur in weld joints during production. Eventually, this informanion will be integrated into an intelligent processing scheme on the manufacturing floor to provide on-line assessment and control of welding quality.

\section{Semiconductor manufacturing}

Sandia has also developed extensive and unique capabilities for simulating and analyzing a wide range of complex chemical processes, such as chemical vapor deposition, that are critical to advanced semiconductor manufacturing. For example, the computer analyzes the interaction between heat transport, fluid flow, and process chemistry for CVD reactors. CVD is used in many areas, from microelectronics device fabrication to production of specialty materials such as ceramic composites. Some manufacturing processes are sensitive to temperature, making computational analysis critical for designing reactors that can control temperature to within a few degrees. Other processes require precise distribution of reactive gases in order to produce highly uniform deposits.

Sandia's Chemkin software is a powerful tool for analyzing surface chemistry and the reacting flow of gases. Other models are aimed at improving the processes for making silicon, silicon dioxide, silicon nitride, silicon carbide, diamond, and gallium arsenide layers for semiconductors. Working directly with microelectronics equipment companies, Sandia is helping to improve CVD reactor performance as well as reduce the time and cost required to develop this equipment.

Computer modeling techniques are also being used to solve problems involving specific pieces of silicon wafer process equipment, to develop contamination-free manufacturing methods that will eliminate defects from inte- grated circuits, and to develop materials that prolong the life of manufacturing equipment. In addition to developing its own computer codes, Sandia is also using commercially available modeling codes, such as FIDAP, to optimize wafer configuration and processing-tool operating conditions. Sandia's work with industrial partners is evaluating the effects of particulate, electromagnetic, electrostatic, thermal, radiation, and light contamination on circuit yield and performance. Such work continues to demonstrate to the microelectronics industry the usefulness of numerical modeling for improving the performance of existing equipment, shortening design times, and avoiding problems before manufacturing begins.

"Currently, computer modeling of manufacturing processes is already reducing the cost and time associated with new product development and experimentation," says Paul Hommert, manager of technology transfer for Sandia's Engineering Sciences Center. "By modeling the processes that industry needs to remain competitive in today's market, Sandia is helping to open the door for US manufacturers to lead the way tomorrow." 团

For more information, call

Paul Hommert, Org. 1502, (5.J5) 844-3449, or Bill Robinson, Org. 8702, (510) 294-3124. 


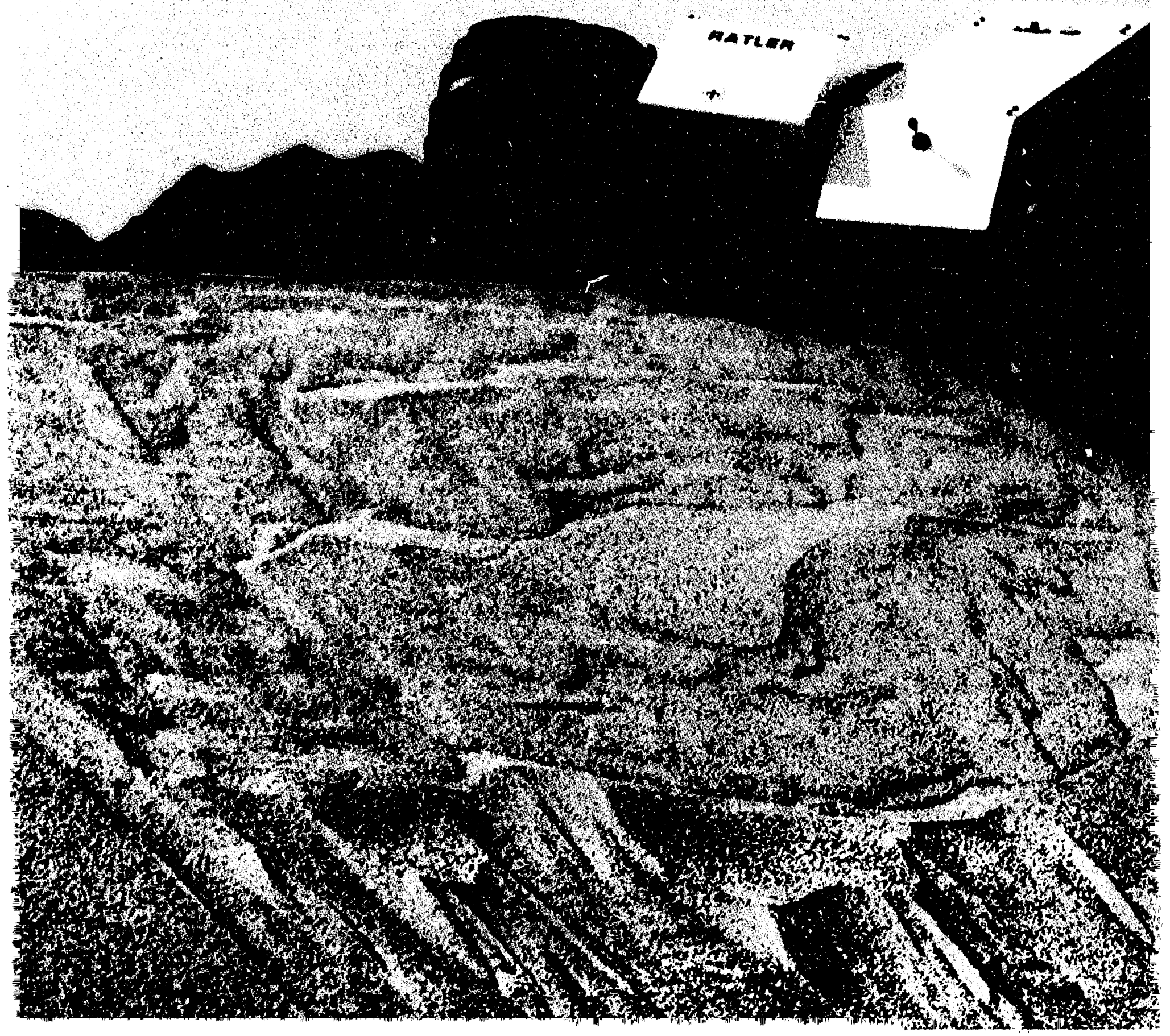




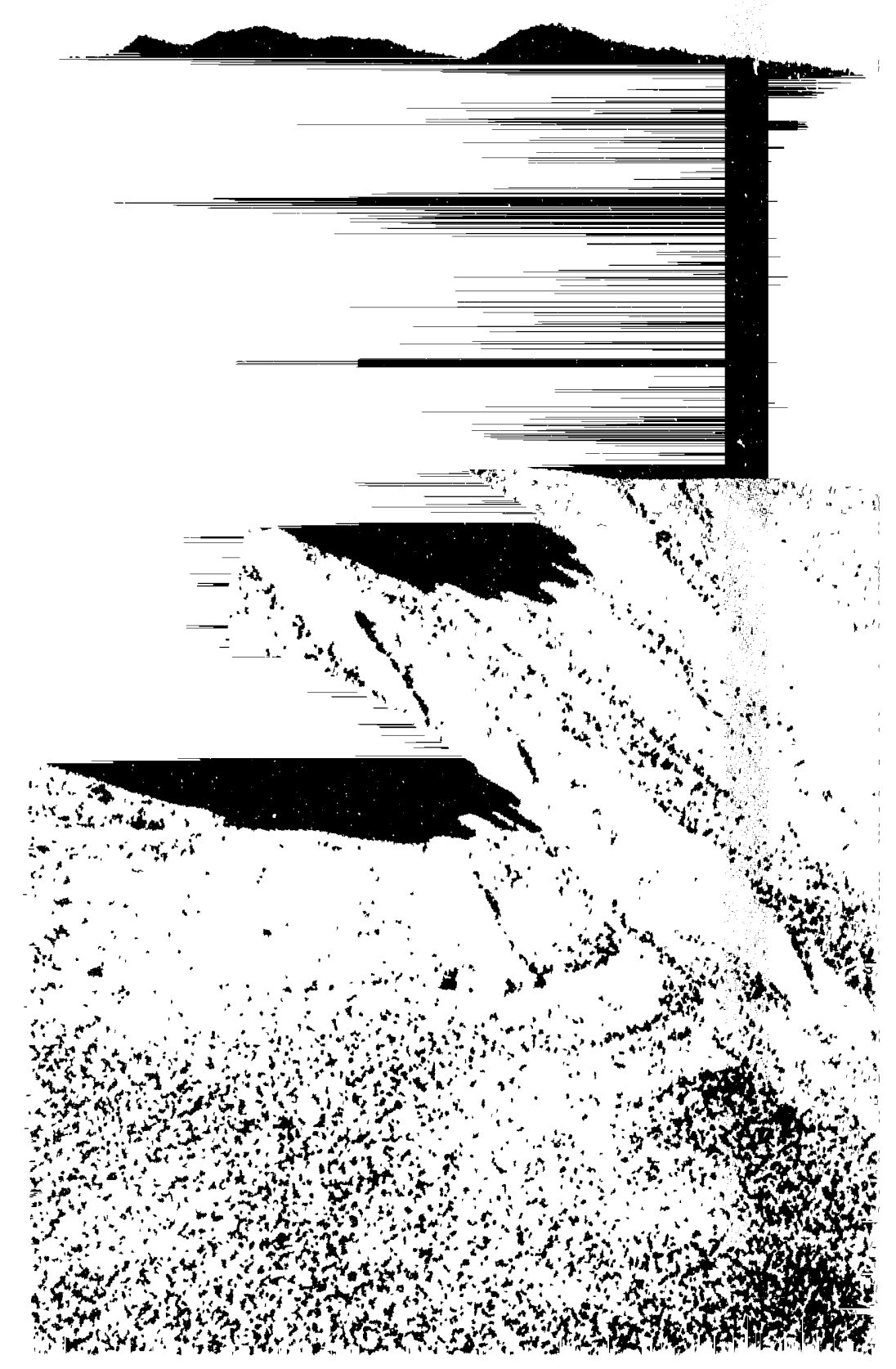




\section{Weapons disassembly}

\section{Robot dismantles nuclear weapons, spares humans exposure to radioactive cores}
$\mathrm{A}$ fter more than 40 years of designing and building nuclear weapons, America's wea- pons complex is now faced with the task of dis- assembling thousands of retired nuclear warheads, a complex and dangerous job.
In the past, this job has been done entirely by hand-by workers wearing protective clothing who follow special safety precautions and change jobs frequently to minimize exposure to radioactivity.
Today, robotic technology is making it possible to disassemble the most radioactive portion of a nuclear warhead without the human touch. Sandia has developed a robot that may soon be used at DOE's Pantex plant near Amarillo, Texas, to remove the plutonium pit - the heart of a nuclear warhead - and place it in a special ship- ping container. All of this is accomplished in the same work space where humans have disassem- bled retired warheads in the past. The robot is a specially adapted, standard industrial robot with computer intelligence provided by Sandia.
"The robot serves as the arms and legs of the operation," says Sandia mechanical engineer Jill Fahrenholtz, project leader for the automated weapon disassembly project. "The warhead is basically a ball of explosives with a plutonium pit in the middle."
The use of robots allows Pantex to cut down the dose of radiation that workers receive, thus keeping employees in specific jobs longer. This reduces the need to continually retrain new employees to handle dismantlement tasks.
The initial stages of the disassembly process that pose little risk of radiation are still done by hand. To start the process, a worker places the weapon on a work surface and removes the outer casing and the first portion of high explosive that surrounds the plutonium pit. After that, the robot takes over, first removing the final piece of high explosive. To do this, it transports the weapon subassembly to a stand that supports the lower half of the weapon shell and injects water around the high explosive to loosen it from the pit inside.
The robot then deploys a vacuum gripper, backed up by safety latches, to pick up the

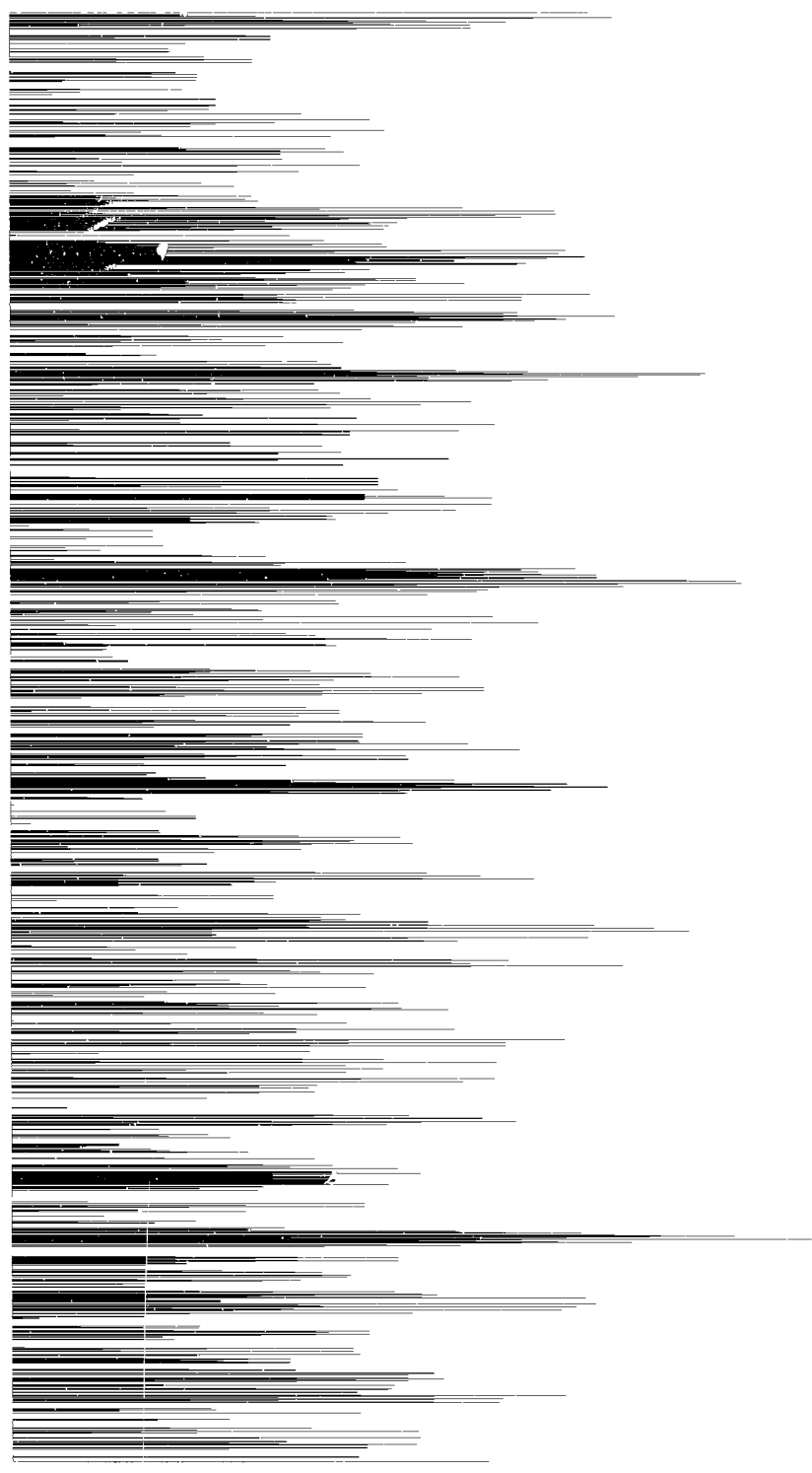

nuclear pit and carry it to a shipping fixture. It picks up the upper assembly of the fixture, attaches it to the lower assembly to cover the pit, picks up an air wrench, tightens the bolts of the shipping fixture, and places a clamp on the upper assembly to minimize vibration. It then uses a mechanical gripper to pick up the whole assembly and place it in an insulated, fire-resistant storage drum.

Because of the delicate nature of the operation, Sandia engineers have designed many safety features as part of the disassembly process. These include hydraulic and pneumatic switches instead of electric switches to completely isolate the warhead from any electric current or shock, as well as insulation between the robot and the 


\section{Intelligent machines can slean up hazardous waste, guard sensitive facilities, explore the moon}

W hether the danger is from hazardous waste, high radiation, or armed attack, robots have a distinct advantage over peoplethey can go boldly where others can't or won't.

Sandia and the Department of Energy are taking advantage of this fact by developing new robotics technologies for a variety of dangerous tasks, from cleaning up buried radioactive waste to exploring the inhospitable environment of the moon. For example, an intelligent machine called RETRVIR (short for Remote TeleRobotic Vehicle for Intelligent Retrieval) can go where humans fear to tread - to areas of high hazard. RETRVIR is a modified all-terrain vehicle that uses a 6-foot robotic arm to dig up buried materials, such as radioactive waste and unexploded ordnance, and place them in special containers.

Sandia is at the hub of a new robotics research initiative that is a collaborative effort of many national laboratories. Sandia was appointed by DOE's Office of Technology Development to coordinate its "Crosscutting and Advanced Technology Program" aimed at developing robotics systems for hazardous waste cleanup. The application of computational simulation and high-performance computing to robot system control is a primary area of research at Sandia.

The new generation of robots uses sensors and automatic planning software to help the robots figure out where to go and how to get there. For instance, although RETRVIR's robotic arm can be manipulated by a human operator through a remote control screen, new software being developed at Sandia is also helping the robot to recognize familiar objects, such as half-buried waste drums, and figure out how to grasp them, clear away the soil, and remove the drums without causing a rupture or spill. A human operator can then simply point to an object on a computer screen, and RETRVIR will autonomously determine how to get to the object and remove it. "Such automated maneuvering can speed up and improve the handling of potentially dangerous materials," notes Peter Boissiere of Sandia's Intelligent Systems Department.

Such advances are made possible by sophisticated mathematical algorithms that enable the robot to choose an appropriate path. In addition, Sandia researchers are developing a navigation system for RETRVIR that will allow it to calculate its own geographic position within 1 to 3 meters by triangulating off Global Positioning System (Gl'S) satellites.

\section{Surveillance and control, lunar exploration}

In addition to RETRVIR, other intelligent machines developed at Sandia include TMSS (Telemanaged Mobile Security Station), known as Thomas for short, and RATLER (Robotic AllTerrain Lunar Exploration Rover). Thomas can be sent to a potentially dangerous site, such as a perimeter fence where armed intruders may be attempting to gain access. "The robot can be equipped with deterrent systems, such as a weapon, a water cannon, or sticky foam, to halt intruders," notes Bryan Pletta, a researcher at Sandia's Robotic Vehicle Range. Thomas can be taught the locations of all roads within a security site, which are then stored in a map database. This enables the robot to automatically select and follow the shortest path to a designated spot within the site. Alternatively, it can be manually guided by a person via radio links that transmit video images and commands.

Thomas is equipped with a video motion detector, a microwave intrusion detector, and a passive, infrared intrusion detector. These sensors, in addition to its computing capabilities, enable Thomas to handle such tasks as detecting intruders, assessing alarms, and responding to alarms.

A robot that has potential space applications is RATLER, a lightweight but strong vehicle made of a carbon composite. Sandia has suggested the use of RATLER for possible lunar or martian exploration. Developed by researcher Jim Purvis, the original RATLER prototype now sits in the Space Hall of Fame in Alamogordo, New Mexico. The robot's sensor package is planned to include a mini-video

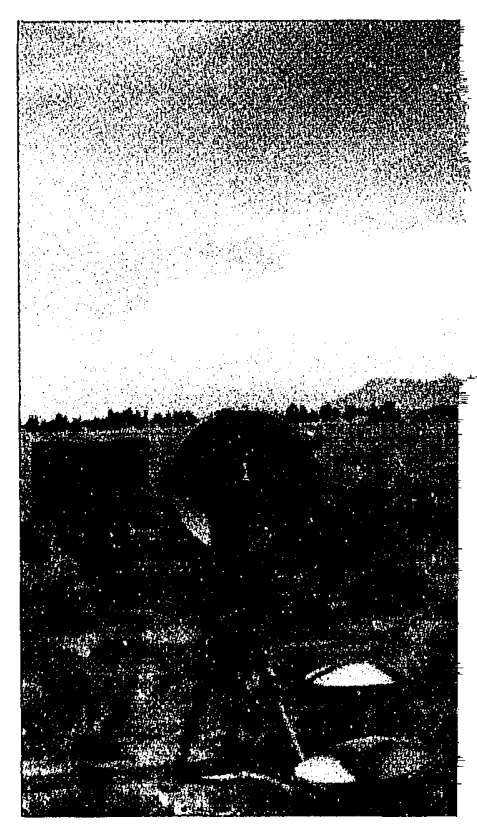


camera and a laser spectroscopy system that can vaporize a target, such as soil on the lunar surface, and take a spectral reading to determine its chemical composition. Martin Marietta is building the laser; Los Alamos National Laboratory is designing the spectrometer. Together, the instruments produce a visual map and a resource map of the surface.

RATLER has a unique chassis that permits all four of its wheels to remain on the ground, even when it is climbing over boulders as high as the diameter of one wheel. This avoids the "fourlegged stool" effect, in which one wheel is suspended in the air, causing the rest of the vehicle to lean to one side and possibly tip. RATLER runs on both batteries and photovoltaics. In addition to demonstrating a prototype of the robot for
NASA in California's Death Valley, Sandia is considering the use of RATLER-like vehicles for such heroic missions as providing access to remote wilderness areas during fires or rescue activities, completing environmental assessment of contaminated sites, handling mining operations, and assisting in battlefield monitoring and military troop support. 团

For more information, contact

Bryan Pletta, ()rg. 9616, (505) 844-5912, or

Peter Boissiere, ()rg. 1661, (505) 845-8966.
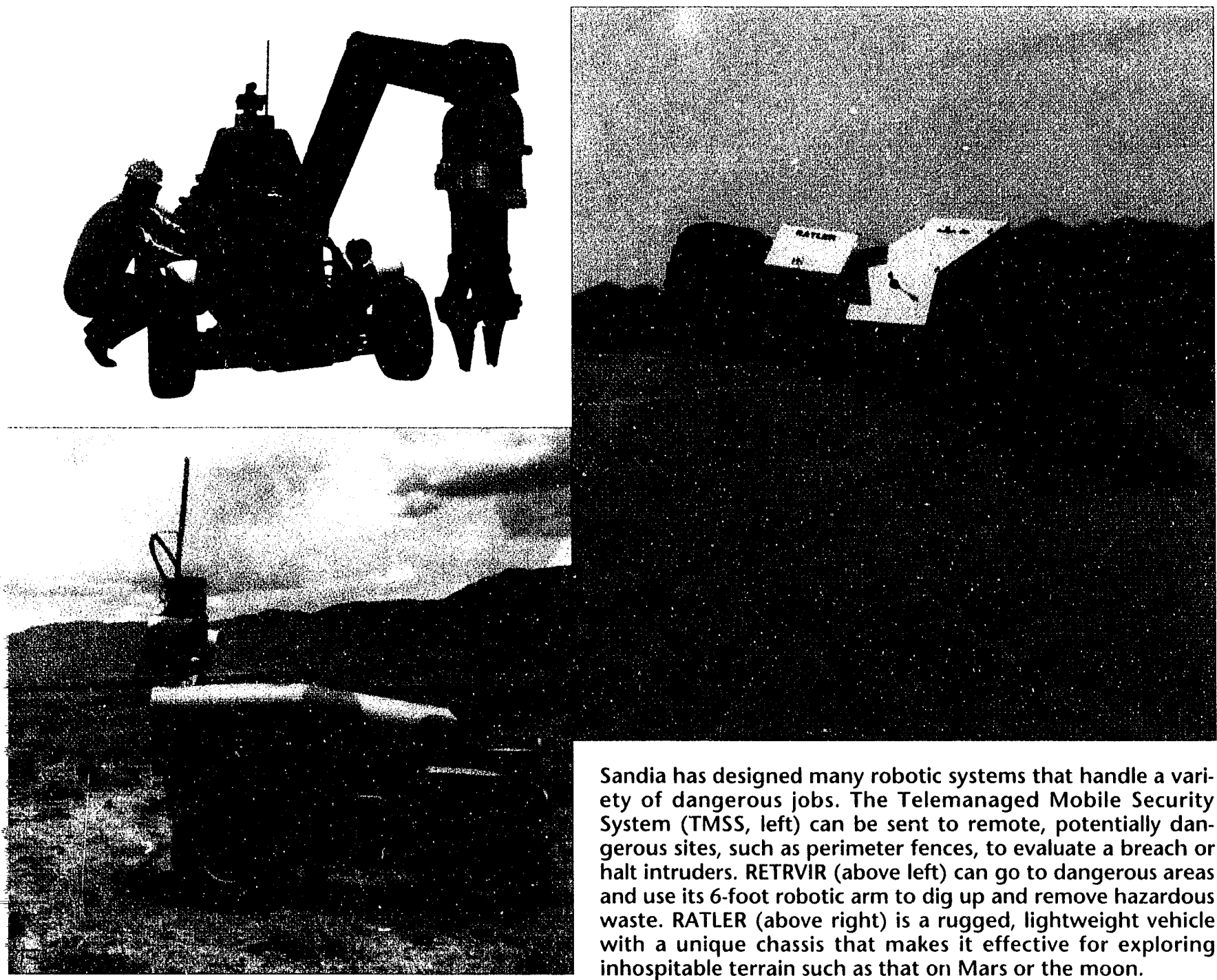

Sandia has designed many robotic systems that handle a variety of dangerous jobs. The Telemanaged Mobile Security System (TMSS, left) can be sent to remote, potentially dangerous sites, such as perimeter fences, to evaluate a breach or halt intruders. RETRVIR (above left) can go to dangerous areas and use its 6-foot robotic arm to dig up and remove hazardous waste. RATLER (above right) is a rugged, lightweight vehicle with a unique chassis that makes it effective for exploring inhospitable terrain such as that on Mars or the moon. 



\section{Super computing in medicine}

\section{Massive memory and computing capabilities can help physicians}

$\mathbf{P}$ ablo Picasso once declared computers useless, saying, "They can only give you answers." No doubt many researchers in the medical field would disagree. In their work, there's no shortage of questions regarding how to optimize human health; any system that can help sift through massive amounts of data to provide answers is extremely useful.

In fact, Sandia is working in several areas with medical researchers to apply high-performance computing technology to improve quality and reduce costs of health care. Sandia's development of supercomputing capabilities for DOE weapon and energy programs now have applications that fall outside the Labs' historical roles.

For example, Sandia researchers, working with Baylor University Medical Center in Houston, Texas, and the Department of Veteran Affairs Medical Center in Albuquerque, New Mexico, have used supercom juters to produce more accurate images of the human breast and brain. Supercomputers have massive memory and computing capabilities that can create detailed, threedimensional images with data collected from magnetic resonance (MR) and other instruments. With higher quality images, physicians are better able to determine the nature of lesions and the extent of any tumors that might be present.

Massively parallel supercomputers overcome shortcomings in available computer hardware and image-processing technology. With conventional MR equipment, physicians are largely limited to viewing a series of two-dimensional "slices" of a three-dimensional object. The massive size of the sets of data acquired in MR scans strain the capacities of even specialized computing equipment. "The fertile ground here lies in using sophisticated image-processing techniques to gain more information than we would have by evaluating the raw data itself," says Dr. Steven Harms of Baylor University.

Sandia researchers programmed their stateof-the-art, massively parallel supercomputers to manipulate and display large sets of data-typically 16 million bytes - from Baylor's MR instruments. One code "slices" through the data, displaying an animated view of each slice as the plane moves through the patient. A second code animates an observed region, allowing a viewer to simulate rotating or ma nipulating a three-dimensional lesion in a breast or the arterial tree of a brain. With this synthetic motion, a viewer can quickly determine the structure of a lesion or arterial stenosis. With just a few minutes of supercomputer computat ${ }_{\text {ion, }}$ a viewer can obtain displays to assess the extent of a tum or with unprecedented accuracy. If a biopsy or lumpectomy were necessa: $y$, geometrical information extracted from the scan could guide a surgeon through the procedure.

Hospitals unable to afford supercomputers might be able to network themselves to other locations with supercomputers. Sandia has executed high-resolution, full-motion medical visualization through a high-speed link over a distance of 19 kilometers. Longer links and data-compression techniques promise to make remote, high-resolution imagery a practical future alternative. The researchers' goal now is to continue to improve the concept and work toward reducing its cost. New directions include work on anaiytical and statistical computations that would analyze multiple MRI scans of the same patier.t over time. Sandia is also applying these analysis techniques to other medical observations such as intravascular ultrasound.

Other biomedical projects at Sandia involving high-performance computing include improving the performance of medical staples used as sutures, developing more potent analogs of the anticancer drug cyclophosphamide, and modeling the chemical and physical properties of chemical compounds used to enhance $\mathrm{x}$-rays and MRI scans. 团

For nore information, call Corrl Diegert, Org. 1424, (505) 845-7193. 

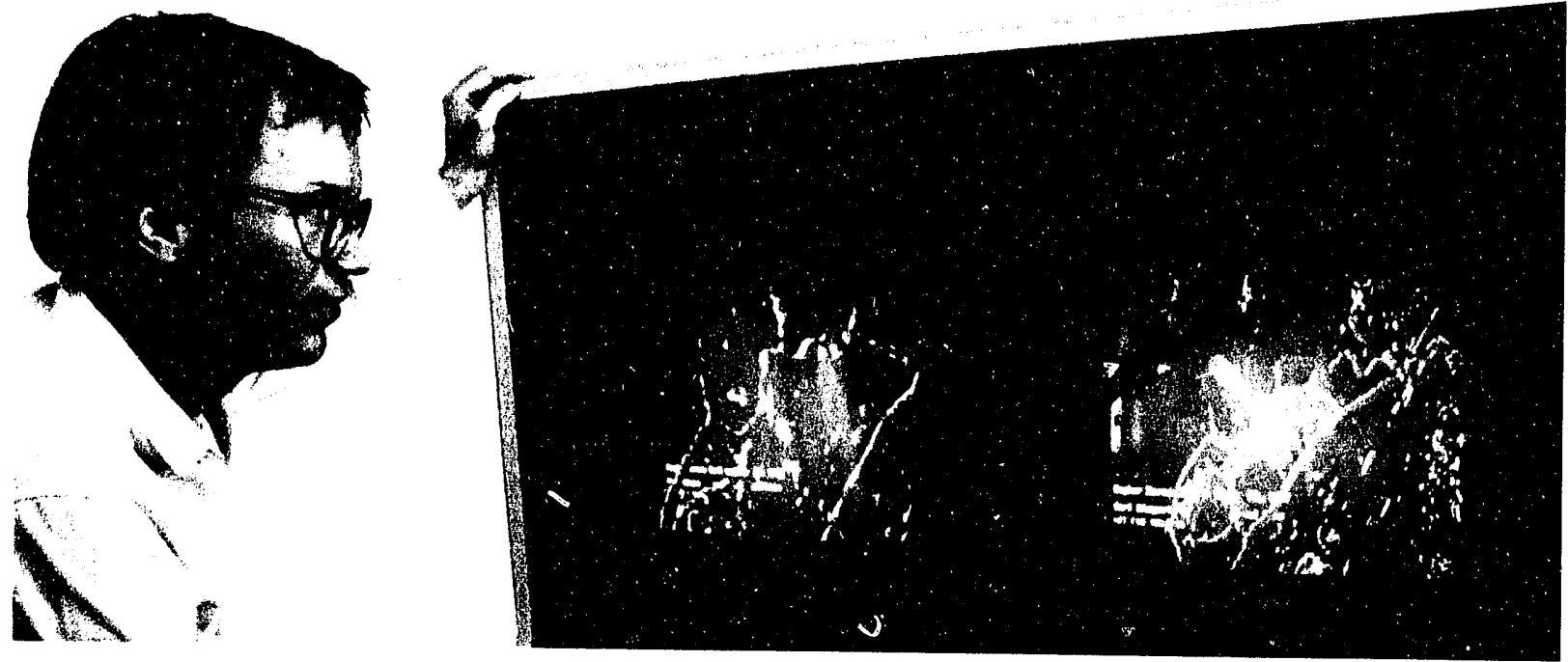

Computational scientist Carl Diegert examines screens showing the data involved in supercomputer analysis of magnetic resonance scans. Displayed on the screens are images of a breast scanned with MR.

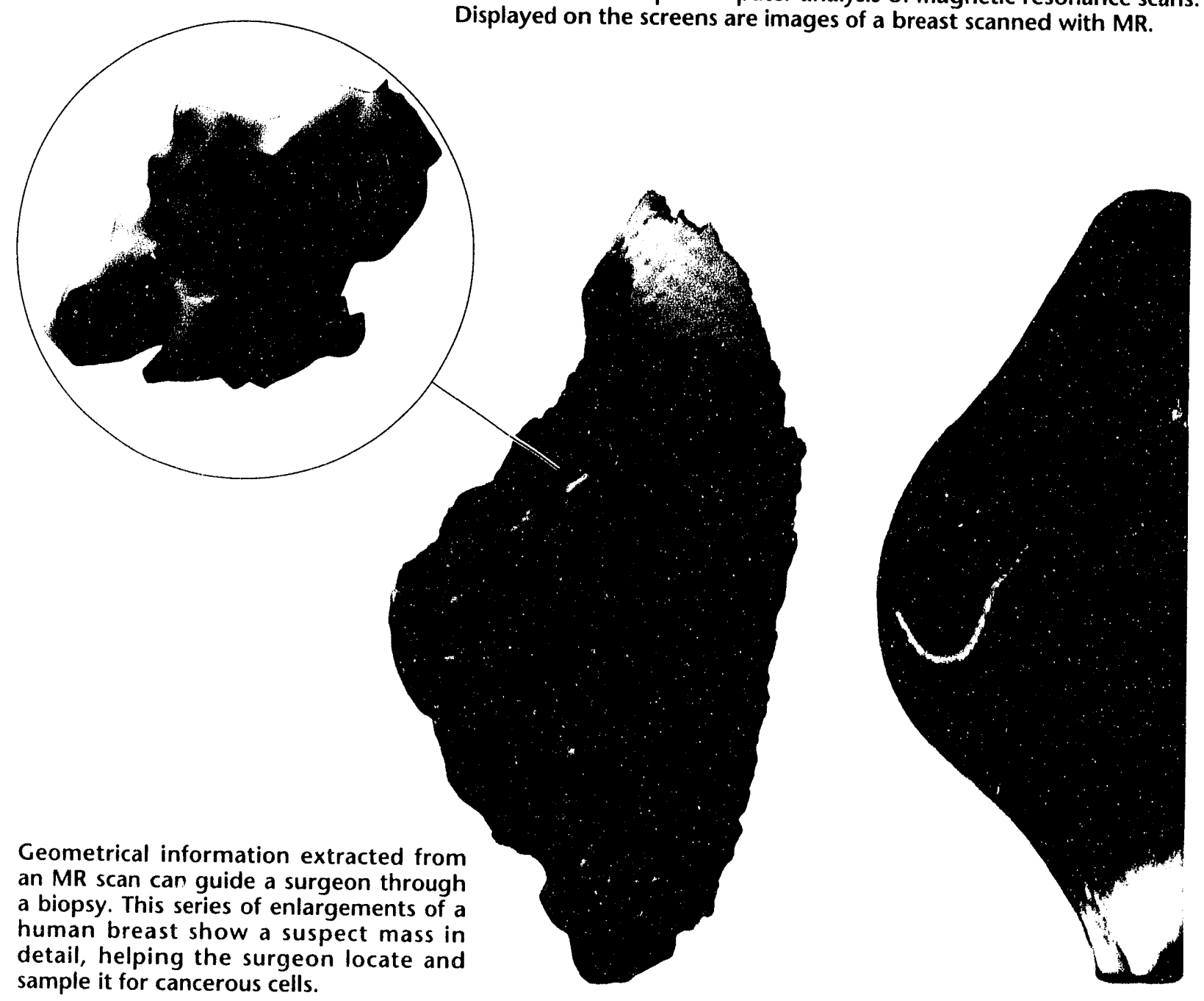




\section{Materials design}

\section{Work with private industry advances knowledge in catalysts for use in engineered materials}

W hile it may seem ironic to some, the largest computers in the world are frequently best used to study some of the smallest units found in nature. For example, Sandia and Biosym Technologies, Inc. have worked together over the past two years on several projects applying high-performance computing to the design of molecules. Their goal: computer-aided molecular design software to aid in developing new engineered materials.
One such collaborative effort between Sandia and Biosym received an R\&D 100 Award in September 1992. Sponsored by the Department of Energy's Offices of Energy Research, Defense Programs, and Conservation and Renewable Energy, Sandia and Biosym are developing new modeling techniques and numerical methods to convert recent theory into commercial software for the molecular engineering of polymer blends and copolymers. Although metal alloys have been used for centuries, the blending of polymers to produce new engineering materials is relatively new. Because of their chain-like nature, polymers mix in a more complex manner than metals. This research focuses on understanding

Sandia researcher Gary Carlson discusses the features of an ethylene molecule absorbed on a 55-atom palladium cluster. Small metal clusters such as this one provide greater catalytic activity than bulk metals. A catalyzed reaction between ethylene and hydrogen will produce ethane.
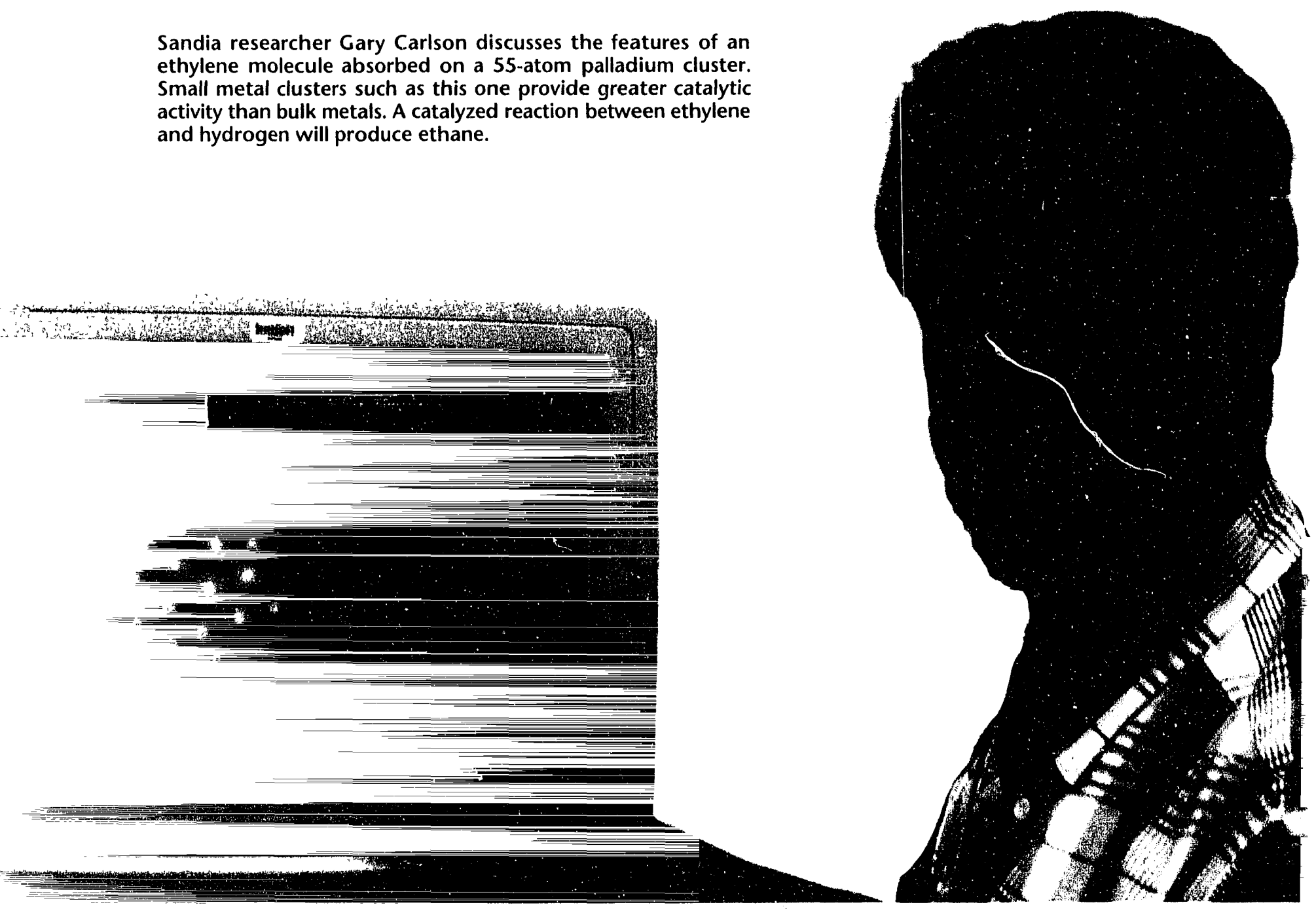


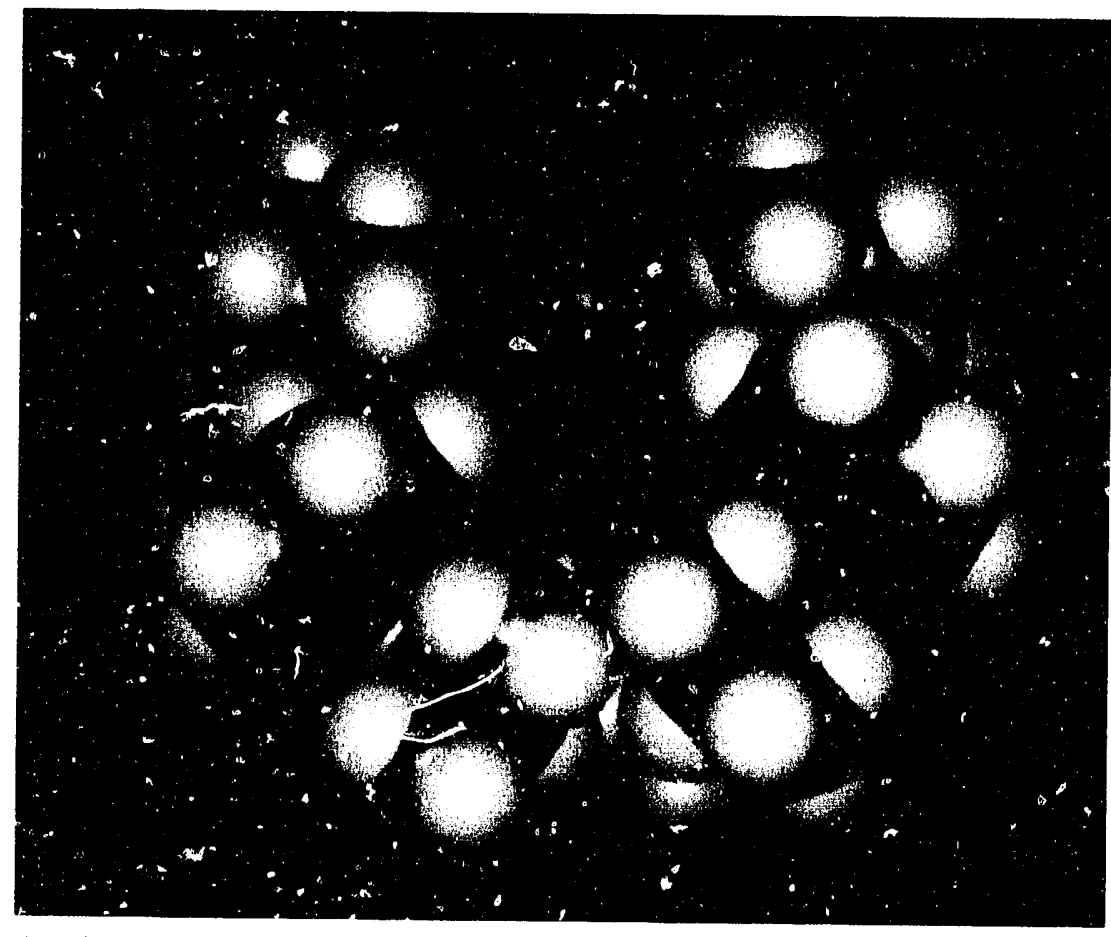

Synthetic metalloporphyrin molecules designed with computer modeling at Sandia are candidate catalysts for converting carbon dioxide to chemical feedstocks. Such catalysts are biomimetic, meaning they mimic nature. Here, a carbon dicxide molecuis is held in the pocket of a nickel octaethyl tetraphenyl porphyrin.

the relationship between the polymer mix properties and molecular structure.

Other collaborative projec: between Sandia and Biosym include biomimetic catalyst design and development of massively parallel computer codes for molecular modeling of cataiytic processes.

Sandia and Biosym have developed advanceci simulation methods for the design and study of biomimetic catalysts based on metalloporphyrins. Metalloporphyrins are organometailic molecules that provide a reactive site in some naturaliy occurring catalysts. By mimicking the structure and furction of the naturai enzymes, researchers can design synthetic versions that can better survive harsh industrial environments.

Another joint study between Sandia and Biosym involves the development and application of massively parallel versions of molecular simulation software. For many problems in molecular simulations, especially those requiring quantum chemical calculations, the speed of available computers is a limiting factor. Massively parallel computers and advanced modeling methods promise to advance significantly the capability to solve these computationintensive problems. Undeı the sponsorship of DOE's High Performance Computing and Communications Initiative and Technology Transfer Initiative, Sandia and Biosym are using Biosym's code and Sandia's massively parallel cornputers to study problems related to chemical and biochemical catalysis.

Sandia and Biosym recognized over two years ago that both institutions had made significant, separate contributions to computer modeling technology and that sharing of these technologies could provide mutual benefits. Through their collaboration, Sandia and DOE learn from Biosym's latest technological advances in molecular modeling; Biosym, in turn, is able to work with top researchers from Sandia on problems of high national interest and to have access to the latest in massively parallel computer technology for its software development programs. 团

Frr more information, call

Gary Carlson, Org. 6211, (505) 844-8116. 


\section{Computing for engineering}

\section{Modeling of semiconductor manufacturing helps improve processing equipment performance}

Semiconductor processing equipment manuSfacturers are faced with a problem: the latest generation of microelectronics devices require processing equipment that performs at higher levels of reliability and quality than ever before. And previously acceptable empirical methods for designing and developing such equipment don't ensure the quality required. Sandia, working with a number of manufacturers, is using numerical simulation techniques to study the behavior of processing equipment and is applying the results of these studies to help improve equipment performance.

For example, Sandia is trying to reduce the buoyancy-induced flow in a lithography tool and is increasing the throughput and uniformity in a single-wafer plasma stripper reactor. f nother modeling effort, through Sandia's Contamination-rree Manufacturing Research
Center. involves applying existing modeling tools to improve equipment performance and serving as a technology clearinghouse for developing new modeling tools to reduce defects and improve future manufacturing capabilities.

Computer simulation and high-performance computing is an area of particular emphasis and expertise at Sandia, involving an interdisciplinary team of more than 500 researchers. Originally developed to support Sandia's work in nuclear weapons engineering and scientific testing, supercomputing applications now have many diverse uses.

To date, Sandia has used the commercially available, finite-element code FIDAP to solve transport equations for operating conditions and geometries defined by manufacturers. The manufacturers also largely define the particular problems that Sandia should work on. To extend its applicability, researchers are modifying FIDAP to allow tracking of contaminant particles and development of modeling tools to treat flows in very low-pressure applications.

These modeling efforts have two primary

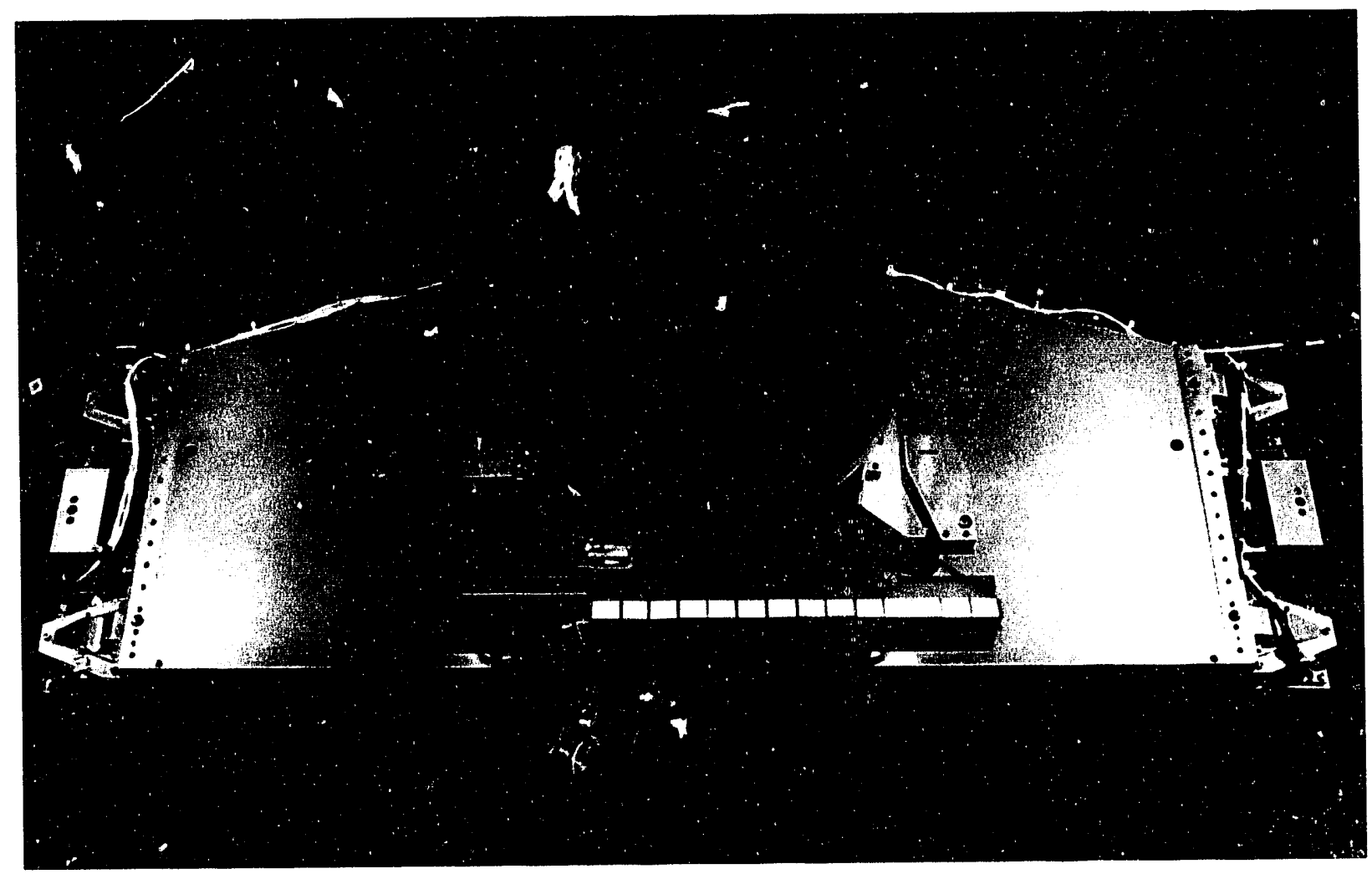

The wafer is positioned on a disk to adjust the position of the wafer each time a new microcircuit is added. 


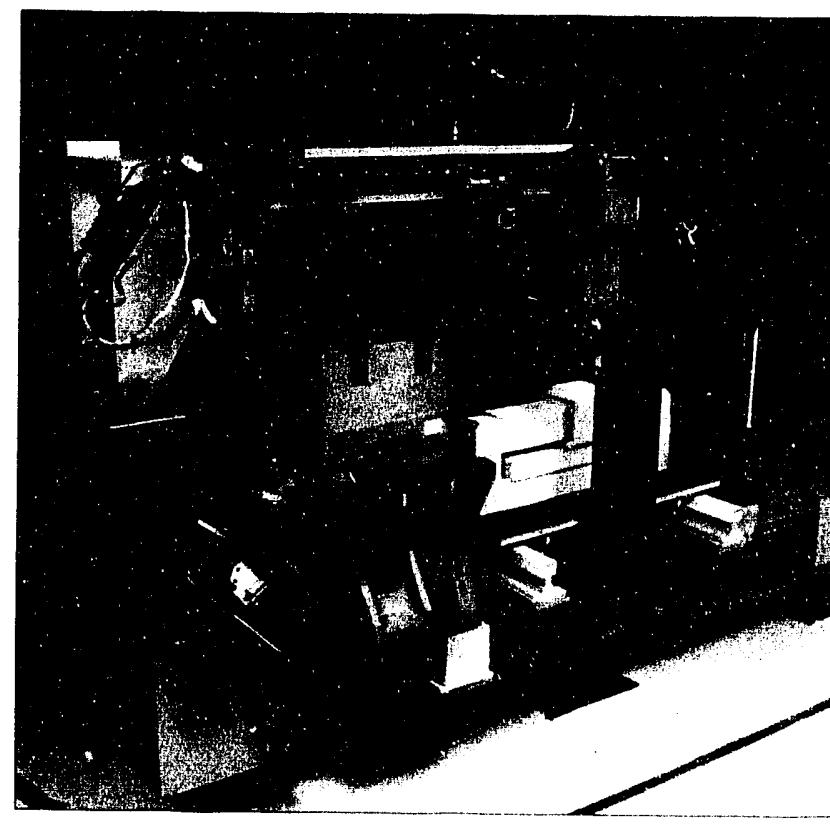

implications. First, by helping equipment manufacturers improve the performance of their tools, the modeling efforts will increase economic competitiveness in the global microelectronics market. For example, one microelectronics manufacturer Sandia is working with hopes to increase profits by $\$ 10$ million by reducing to 20 percent the number of killer particles per wafer - that is, microscopic bits of debris that impair electronic circuits.

Second, these efforts are demonstrating to the microelectronics industry the usefulness of numerical modeling in improving the performance of existing equipment and also showing how design times can be shortened and problems avoided before manufacturing begins.

Through participation in a SEMATECH chemical-vapor-deposition modeling workshop, Sandia helped demonstrate how numerical simulation could improve the design of microelectronics process equipment. Numerical modeling has improved the design of the flow distribution system in a plasma stripper reactor. The results of mode'ing the design yielded insight into the behavior of the flow through the distribution baffles, which were not available previously, and allowed a new baffle to be designed that improved etch uniformity. 团 


\section{Radiation modeling}

\section{Sandia-designed computer program models penetration of photons, electrons}

$\mathbf{R}^{\mathrm{a}}$ adiation is present in many places in modern society - in space, in the atmosphere, and in nuclear reactors. Radiation is produced by $\mathrm{x}$-ray machines and nuclear weapons. Radiation is helpful in some cases - if it kills harmful cancer cells, for example-but dangerous if it damages healthy cells.

Because a little radiation goes a long way, precise use of radiation is critical. To improve precision, Sandia has developed a radiation modeling capability to ensure effective and safe use of radiation.

Over the years, with funding from the Department of Energy, Sandia scientists and mathematicians have developed a suite of computer codes for modeling radiation. Although Sandia's primary interest has been in modeling the vulnerability of weapon systems to radiation, the computer codes have also been placed in the public domain and have found applications in such diverse areas as medicine, food preservation, electron beam welding, nuclear reactor safety, and radioactive waste cleanup.

The latest compilation of these codes, made publicly available by DOE in 1992, is Version 3.0 of the Integrated-TIGER-Series, or ITS. This is a system of general-purpose computer codes that have been distributed worldwide for the past two decades. Users of the system obtain it through DOE's code center at Oak Ridge National Laboratory. The new version is more efficient, easier to use, and is based on a more accurate physical model than previous versions.

"We also added macroscopic effects of magnetic and electric fields," says John Halbleib, a Sandia physicist who helped write the codes. "We were primarily interested in the vulnerability of electronics in Sandia-designed weapon systems."

"The codes have other uses as well," notes nuclear engineer Ronald Kensek, who also helped design the codes along with researchers at the National Institute of Standards and Technology. "We use them to model the energy deposition of radiation sources. The system has been a useful tool for inventorying radioactive waste, for example, or for determining the depth of heating

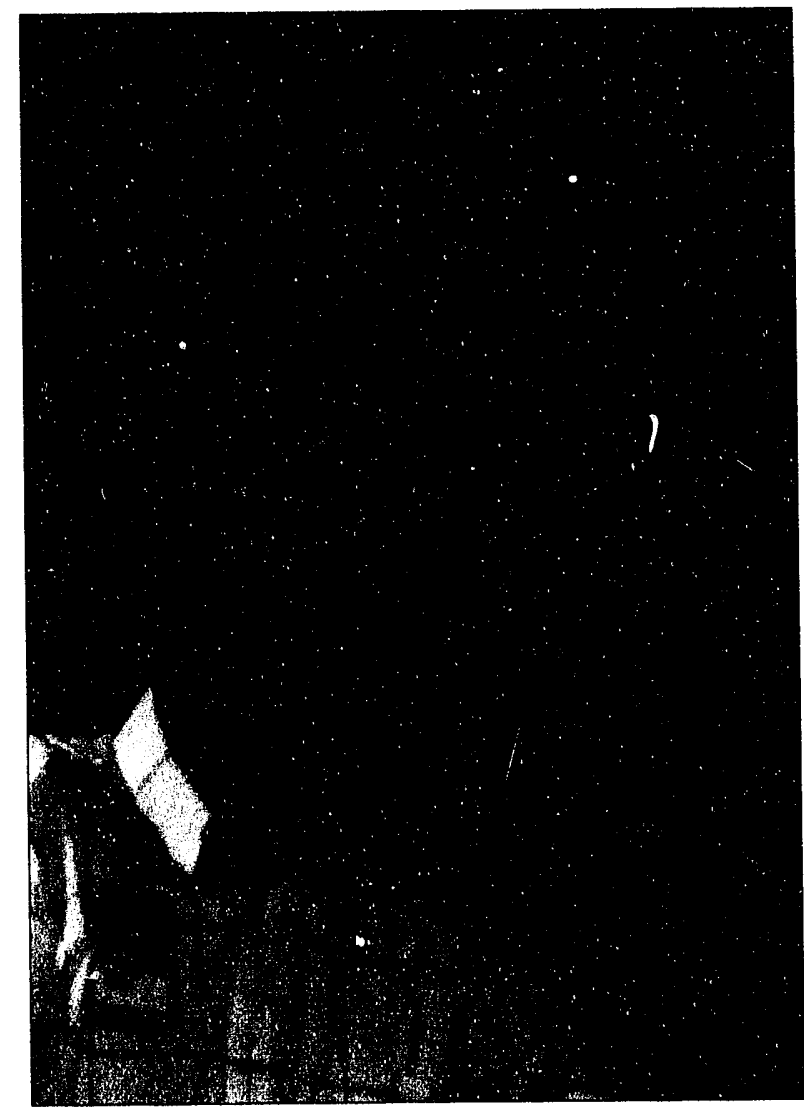

in a metal joint that's being welded with an electron beam."

\section{Codes have many applications}

Based on mathematical probability, the codes follow a random individual particle, such as a gamma photon, as it collides with other materials or particles to see how far it penetrates a given material. For any particular study, the code may calculate millions of such histories and then calculate an average.

Since their inception, the ITS codes have had versatile applications. They were used to design the electron telescopes on Voyagers 1 and 2 on their odysseys through the solar system. They were used to do shielding calculations for Sandia's Particle Beam Fusion Accelerator (PBFA), a facility dedicated to achieving nuclear fusion in the laboratory. For instance, when Sandia researchers wanted to build an $\mathrm{x}$-ray pinhole camera for PBFA, they needed to know how much shielding to use to prevent particles from scattering and overexposing the image. The ITS computer model accurately predicted that scat- 
tering would be sufficiently low so as not to be a problem.

Medical uses for the codes include cancer research and imaging of vascular systems. Nuclear scientists from Europe and the Nuclear Regulatory Commission have used the codes to study the reliability of critical electronic subsystems in nuclear reactors. Weapon designers have used the system to study the range of particle energies emitted by nuclear weapons. In fact, the codes were primarily designed by researchers involved in simulating radiation eftects iil aboveground and underground nuclear testing.

One of the more unusual applications of the codes has been to study a $198 \%$ supernova by determining the concentration of intermediate atomic-number isotopes emanating from the explosion.

Other uses include transportation of radioactive waste, extent of contamination in radiation cleanup, research into the cost-effectiveness of radiation sterilization of hospital waste, the study of Van Allen belt radiation, sterilization of meats to extend shelf life, and particle-induced, singleevent upsets of microelectronic circuitry.

Future developments of the code will include combining it with another soon-to-be-released code known as ADEPT to provide an even more powerful three-dimensional capability. 田

For more information, call

John Halbleib, Org. 9341, (505) 845-7064, or

Ronald Kensek, Org. 9341, (505) 845-7642.

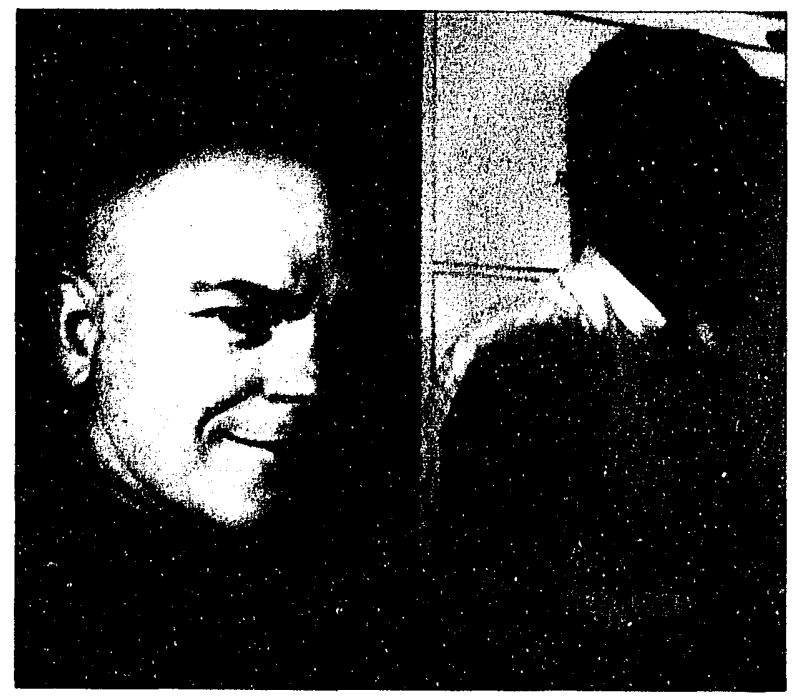

Computer codes developed at Sandia model the effects of radiation and have been placed in the public domain for use in such diverse areas as medicine, nuclear reactor safety, electron beam welding, and radioactive waste cleanup. Developers of the most recent compilation of these codes include (this page, from left) John Halbleib, Greg Valdez, and Ronald Kensek (facing page). 


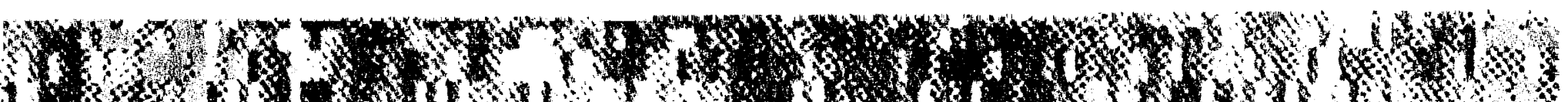

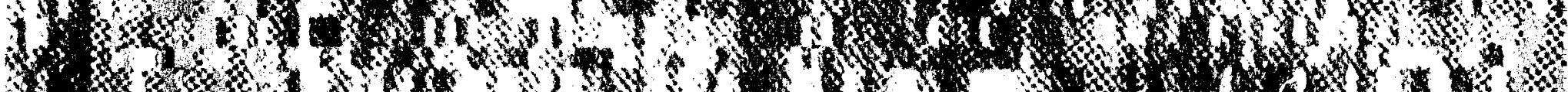

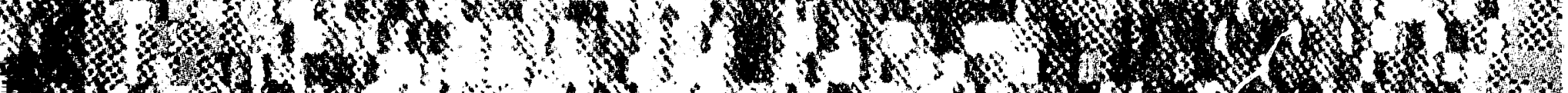

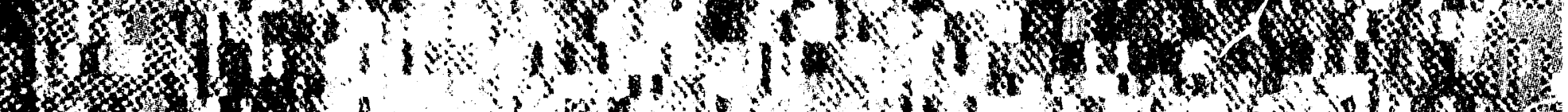

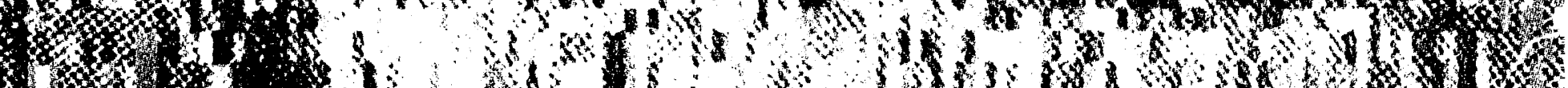

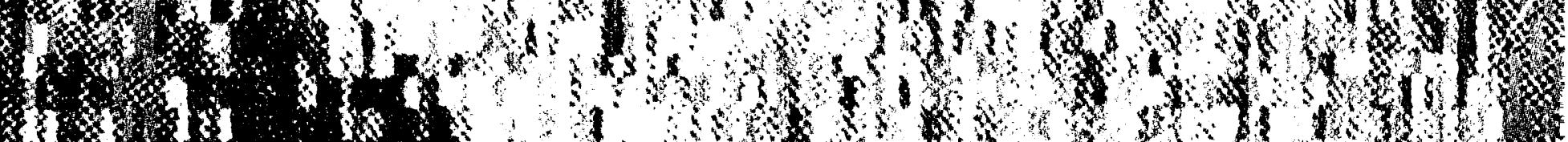

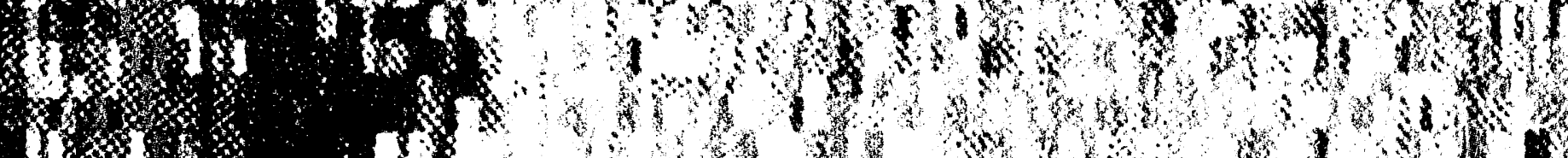

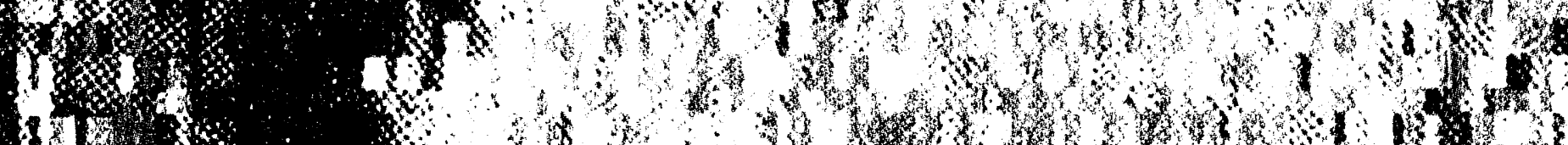

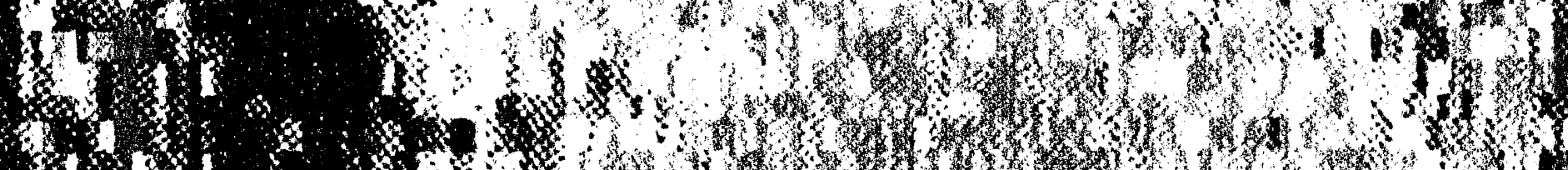

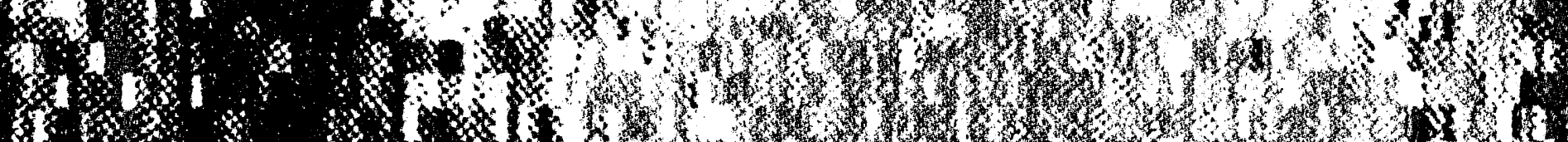

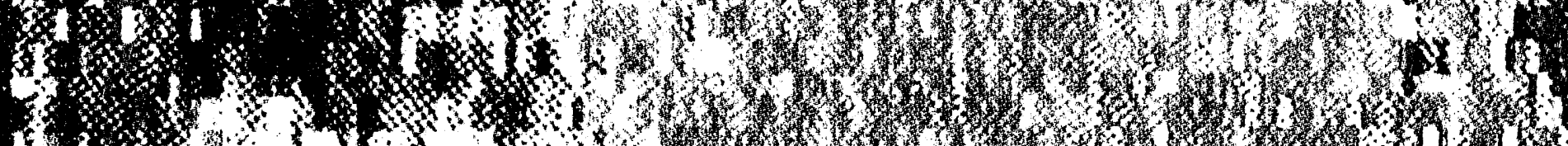

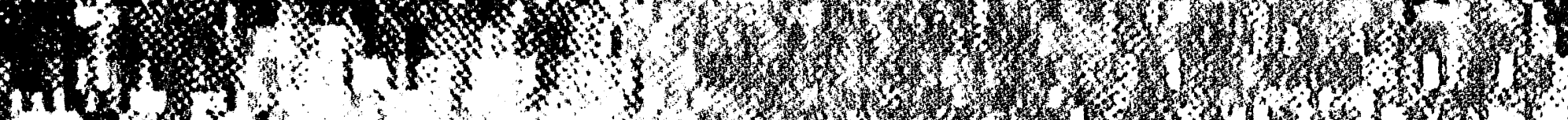

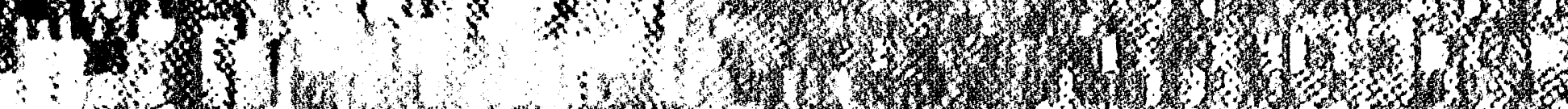

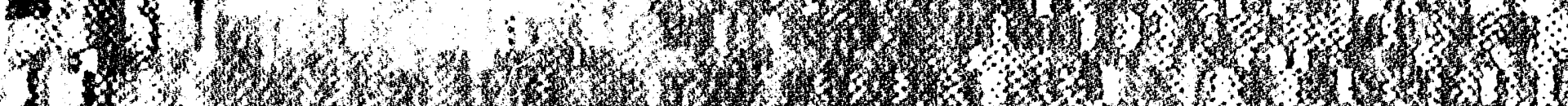

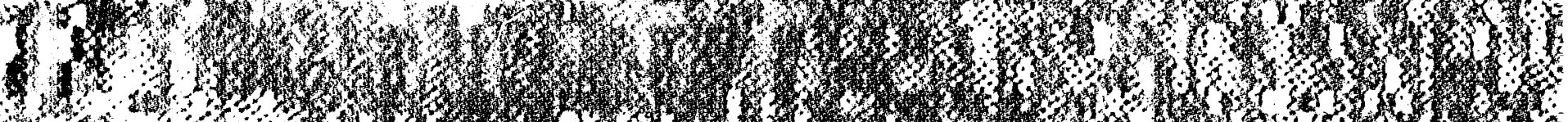

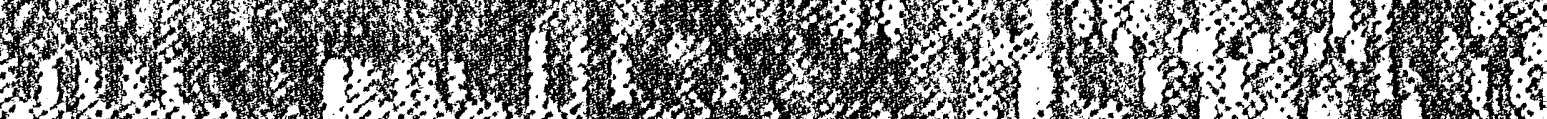
(1) 洒

, Cr. $3 x^{2}+x_{2}$ .

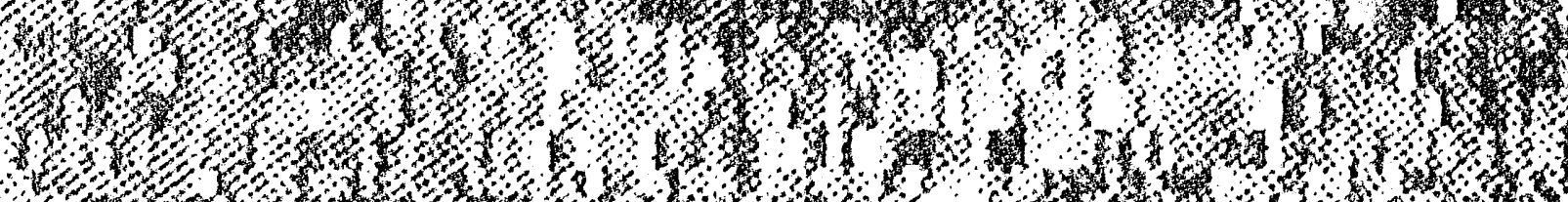
J 7. . . (3) -

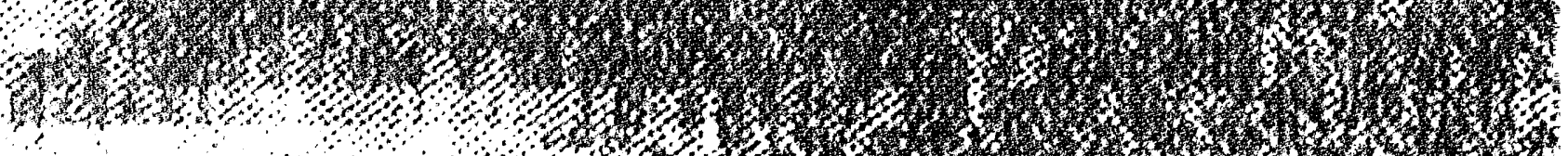
. (1)

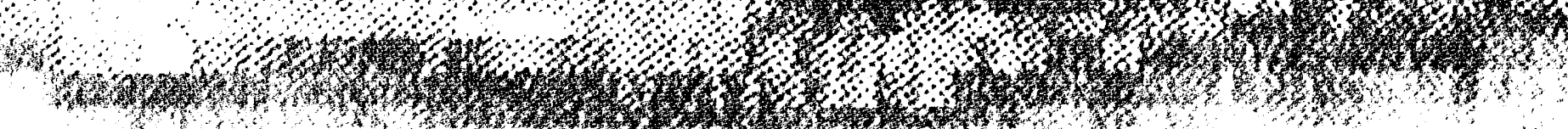
\%ow

- now on 


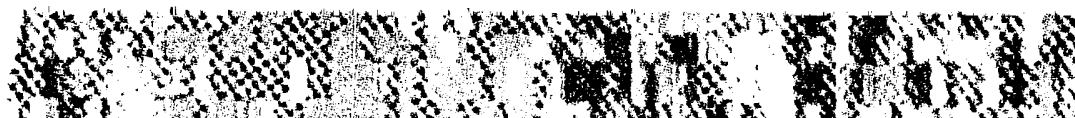

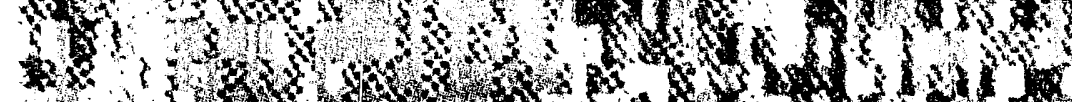

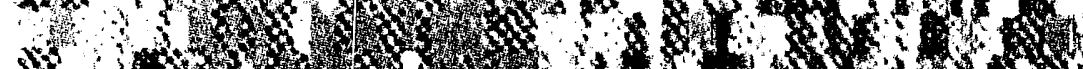

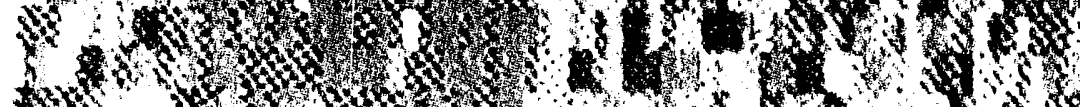

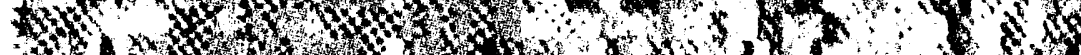
H. (3)

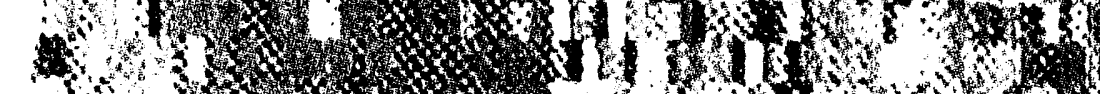

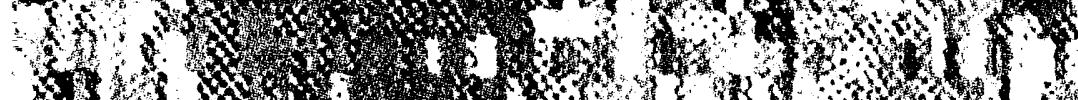
7.

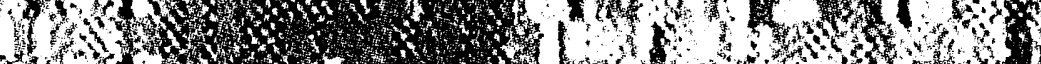

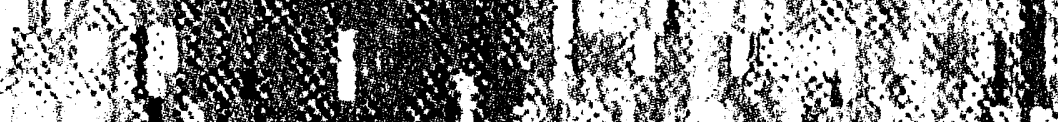

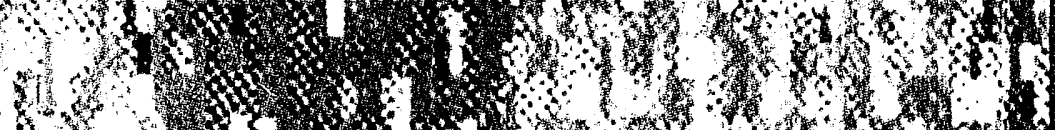

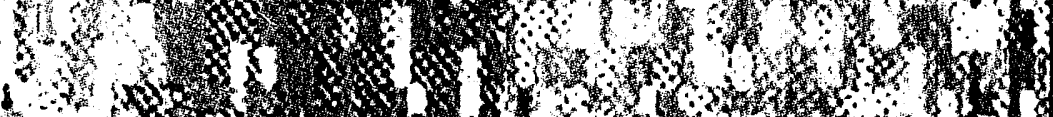

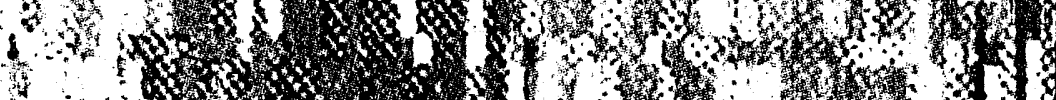

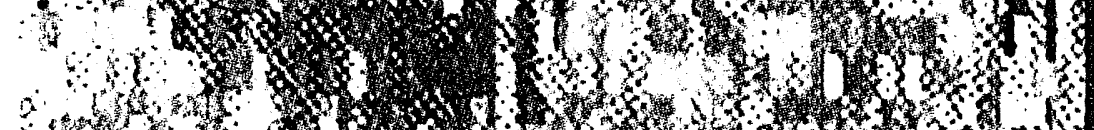

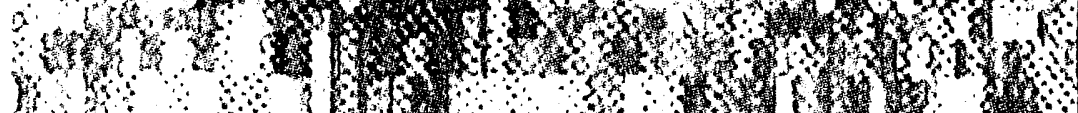

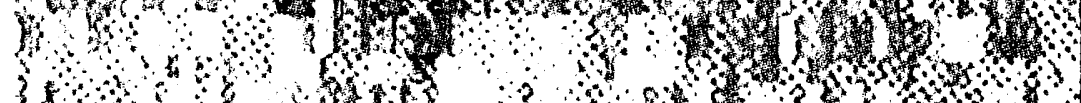

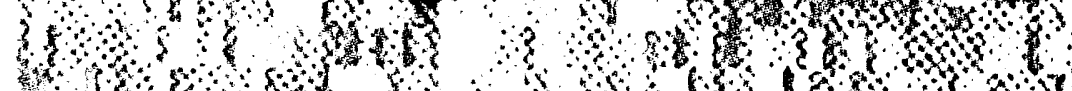

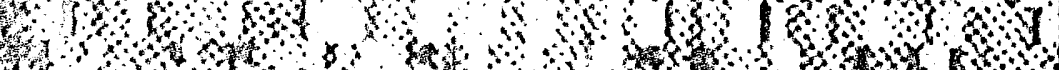

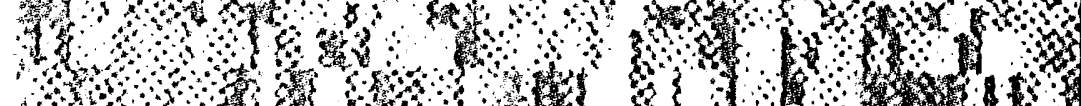
कow

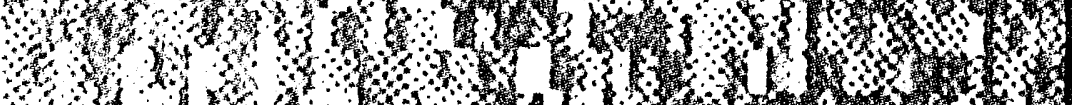
H.

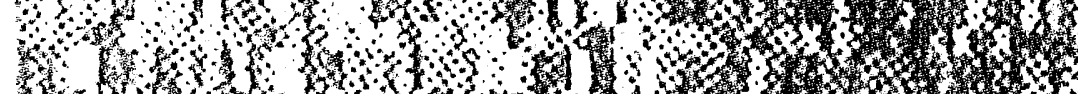


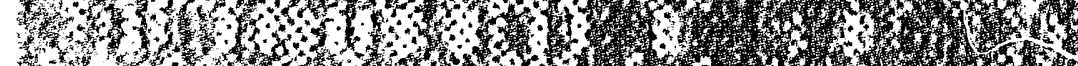

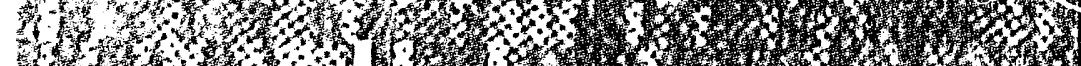

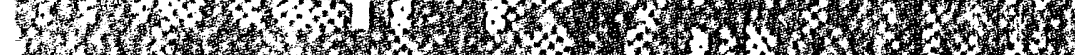

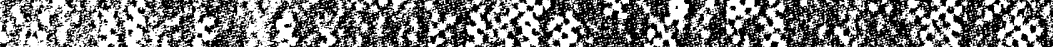

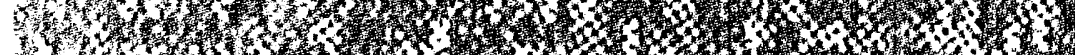

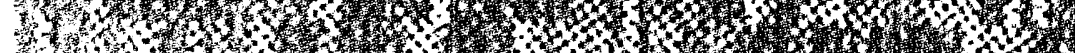
1,

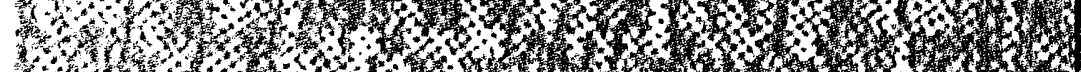

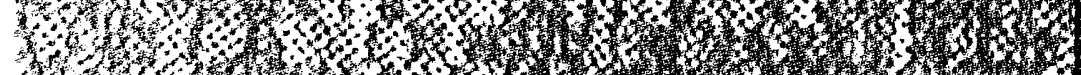

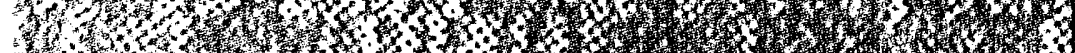

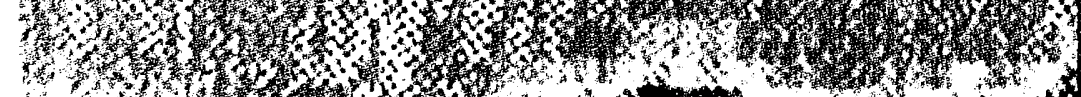

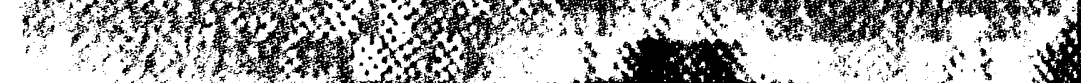

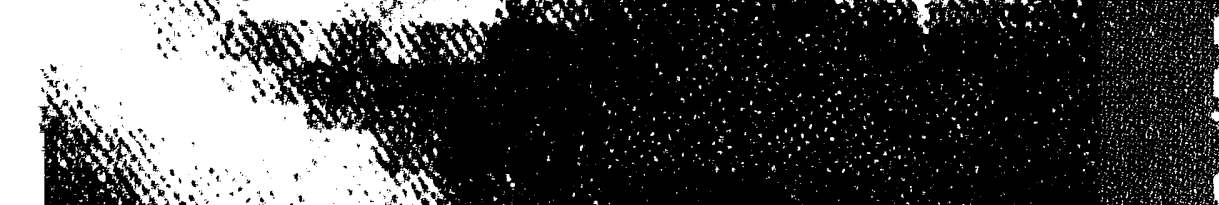

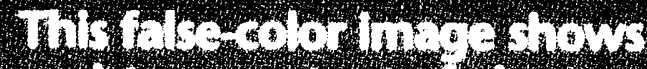

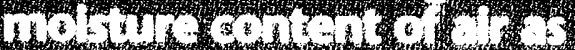

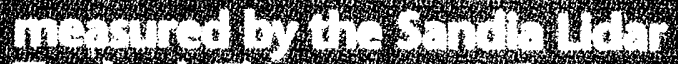

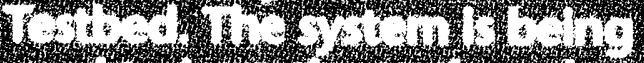

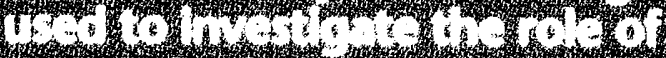

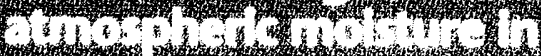

W. W

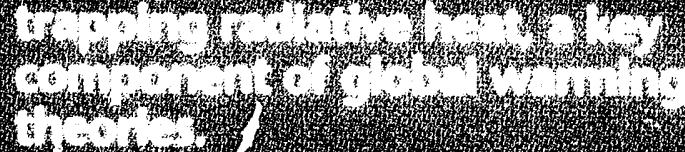




\section{Glucose monitor}

\section{People with diabetes could say good-bye to blood samples}

$\mathbf{E}_{\mathrm{f} e \mathrm{rag}}$ very now and then, a technology developed for a completely different purpose ends up changing the lives of millions of people.

Such a technology is the noninvasive glucose monitor, developed by Sandia and the University of New Mexico School of Medicine. It has the potential to eliminate daily, needle-drawn blood samples for an estimated 2.5 million people with diabetes in America. By expanding on infrared spectroscopy and advanced statistical techniques originally developed to analyze aging explosives in nuclear weapon systems, this ingenious device has the potential to completely alter the way patients track their blood sugar levels.

It also has the potential to improve the treatment of diabetes. Medical doctors, like UNM's Ries Robinson, will attest that one of the ongoing challenges in treating diabetes is making sure patients test themselves often enough, which can be as many as four or more times a day.

"The enormous costs and life-threatening complications that can result from this disease, and the daily discomfort involved in its care and treatment, make this technology a tremendous boon for diabetics," says Robinson, who helped develop the non-invasive sensor. Robinson is also the first resident of UNM's School of Medicine to receive part of his training as a member of a Sandia research team.

Each year, 12,000 Americans lose their sight to diabetes, the leading cause of blindness in people ages 25 to 74 . An estimated $\$ 20$ billion is spent annually for care and treatment of the disease and its potentially fatal complications, such as kidney disease, amputations, and increased risk of heart disease and stroke.

\footnotetext{
A light approach to monitoring glucose Researchers are optimistic that a commercial glucose monitor would be both painless and accurate. It shines infrared light -in this case, near-infrared, which is only slightly longer in wavelength than visible light - through a finger. The light transmitted through the finger is measured and analyzed to determine glucose concentration based on the principle that, like all substances in nature, glucose absorbs light at specific wavelengths.
}

Although the technology is still in its infancy, it has already been tested on human patients in the laboratory and has proved to be sufficiently precise for making clinical decisions. Sandia hopes to pursue the goal of developing a convenient, cost-effective home monitor. A future goal is to develop a portable monitor that can be coupled with a programmable insulin pump. An implantable, miniaturized insulin pump, developed by Sandia and UNM in the 1970s and transferred to industry, is now being tested in about 700 patients worldwide.

Sandia chemist David Haaland, who also helped develop the glucose sensor, notes that the new technique has the added advantage of providing continuous monitoring, as opposed to timebound, momentary information about fluctuations in blood sugar levels. This can be especially useful for patients who are undergoing surgery or childbirth, when their blood sugar levels must be constantly monitored.

"Unlike more conventional technologies, we are able to simultaneously analyze the entire spectral region containing a multitude of data points," says Haaland. "This translates into greater precision, sensitivity, and reliability in the sensor."

Differences in skin pigmentation, blood type, and finger thickness, which could affect the spectral readings, are accounted for in a sophisticated algorithm that analyzes the spectral data.

Researchers at Sandia and UNM are considering the potential for using the same technique to measure other biological substances, such as blood alcohol and cholesterol.

Their work is part of a collaborative research effort that has been funded by both Sandia and UNM. Testing of the monitor on patients has been conducted at the UNM School of Medicine's Clinical Research Center. This center is supported by the National Institutes of Health. Sandia's work has been funded through its Technology Maturation Program.

In fact, as Robinson points out, the glucose monitor demonstrates the kind of technological breakthrough that is possible when two diverse public institutions team their capabilities. In this case, Robinson devoted one-third of his time to caring for patients at the UNM hospital and twothirds of his time doing spectroscopy research in a Sandia lab - a rather innovative collaboration among medical school research programs. 团 
For more information, call

Ries Robinson, UNM School of Medicine,

(505) 277-3881, or

David Haaland, Org. $1823,(505) 844-5292$.

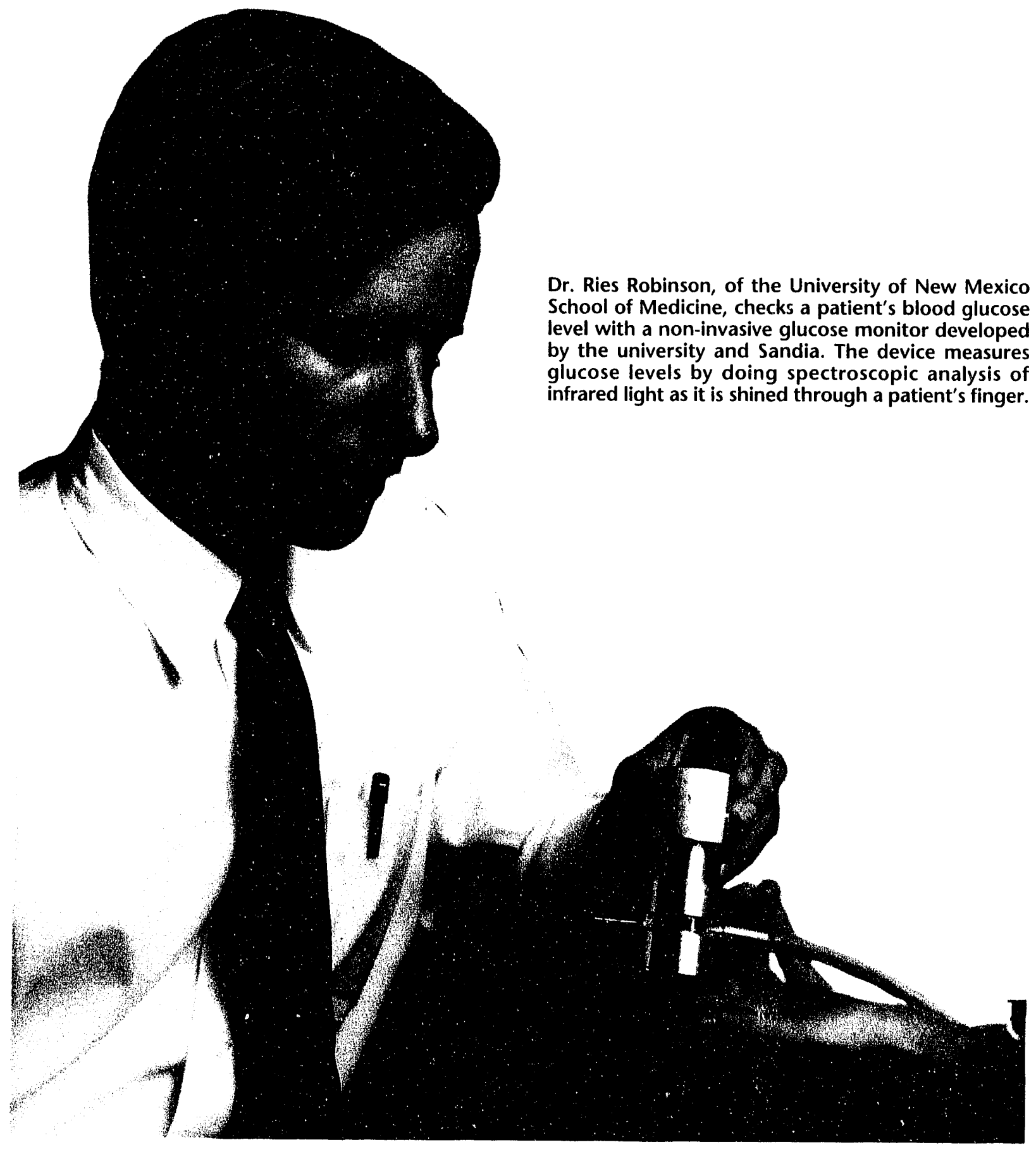




\section{Hydrogen sensor}

\section{State-of-the-art microelectronic device detects hydrogen leaks, improves safety}

$\mathbf{T}$ The most abundant element in the universe is also one of the more explosive. If it comes into contact with a spark in the presence of oxygen, it rapidly ignites.

Fortunately, in the Earth's atmosphere, hydrogen gas is too dilute with other elements such as nitrogen to create a cataclysm. But in 's pure form, it has many industrial applications, ranging from the manufacture of ammonia and methanol to the desulfurization of petrcleum products. Liquid hydrogen serves as fuel for the space shuttle.

In environments such as these, manufacturers must monitor frequently for hydrogen leaks, an expensive and time-consuming process. According to the National Aeronautics and Space Administration, hydrogen leaks are one of the primary causes of delays of space shuttle launches.

In the past, hydrogen detection systems have either been extremely large and expensive, such as those using mass spectrometry, or slow and less than accurate, such as those involving grab bottles with lids for capturing air samples.

But now Sandia has developed a microelectronic hydrogen sensor capable of detecting

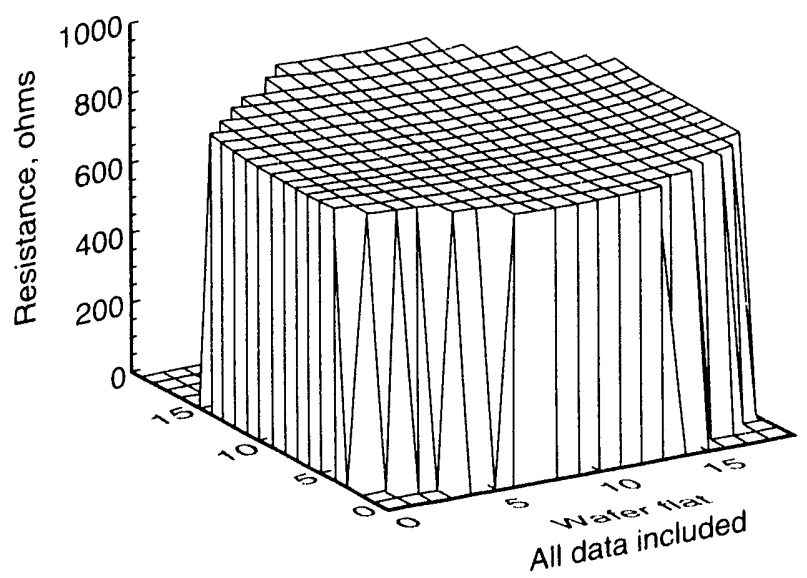

hydrogen over a broad range of concentrations, from 1 part per million to 100 percent. The sensor is small enough to be placed at many various points, each collecting data simultaneously. Once it is fully tested and placed on the market, the sensor is likely to make existing techniques obsolete because it is smaller, faster, more rugged, and less expensive to manufacture.

"It's really an amazing sensor because of its wide dynamic range and low cost," notes Paul McWhorter, a Sandia electrical engineer who helped design the hydrogen sensor. "You get the same performance, high reliability, and low cost that you would expect from modern microelectronic devices, such as fast memory chips in computers."

The sensor has many potential applications, including detecting hydrogen leaks on space orbiters, monitoring hydrogen buildup in radioactive waste tanks, detecting impending transformer failure in electric power plants, and monitoring hydrogen buildup in plutonium reprocessing.

\section{State-of-the-art microelectronics technology}

The sensors are manufactured on 6-inch silicon wafers in a Class I clean room using state-of-theart microelectronics fabrication techniques, an area of demonstrated technical competency at Sandia. Microelectronics research is a key area

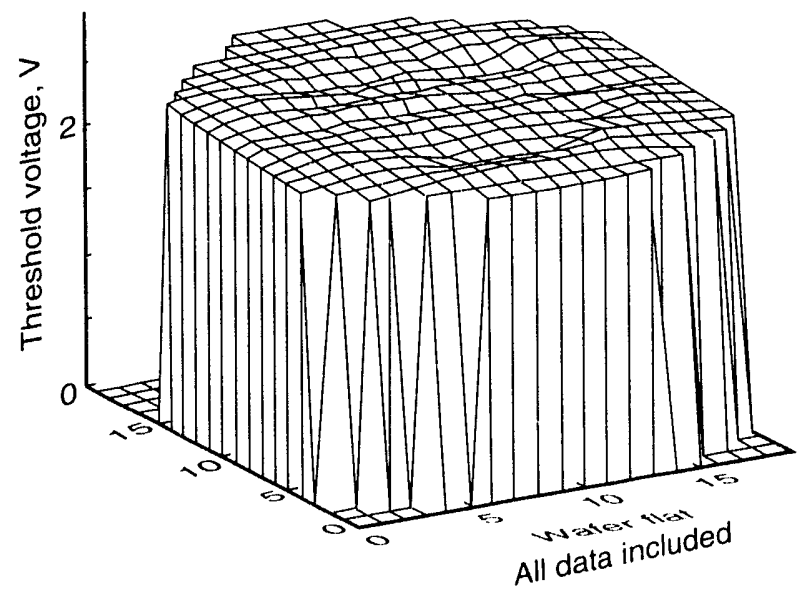

The hydrogen sensor developed at Sandia is both reliable and manufacturable, as shown in these graphs measuring hydrogen detection performance. The smooth surface at the top of the graphs shows the consistency of the results obtained with both the resistor sensor and the transistor sensors. 
of concentration at Sandia and is supported by the Department of Energy.

A Class l clean room contains, on average, fewer than one half-micrometer-sized particle per cubic foot. In this environment, which is free of lint or other airborne contaminants that can impede the performance of extremely small components, 500 sensors can be built on a single wafer; 20 wafers can be fabricated in a single manufacturing run, producing a total of 10,000 sensors and driving production costs down.

The Sandia system actually combines two kinds of sensors, a resistor and a transistor, both of which react with hydrogen to change their material properties. In the resistor sensor, electrical resistance increases as hydrogen levels rise. This is because the resistor absorbs hydrogen atoms, scattering electrons. In the transistor, the threshold voltage decreases as hydrogen levels increase.

NASA is testing the sensor system on a prototype turbopump for the space shuttle engine at its Stennis Space Center in Mississippi. The space agency currently uses a sensor system that detects hydrogen over a broad area such as a launch pad but cannot pinpoint the source of a leak.

"This is the first demonstration of a multiple-point hydrogen detector in a field application," says McWhorter.

An added feature of the sensors is that each one is equipped with its own intelligent heater and eight thermometers. "One of the neat things about this system is it's smart enough to maintain its own temperature, which keeps the readings from going out of whack," says project leader Jose Rodriguez.

"Let's say we're looking at a leak detector at a joint in the space shuttle hydrogen tank," he explains. "Hydrogen starts flowing into the tank, causing the joint to cool down, and the sensor starts cooling as well. Because it has to have a constant temperature in order to operate accurately, the sensor automatically engages power to its on-board heaters to keep itself warm."

The specific operating temperature is flexible, but it must remain constant for the measurements to be accurate. At higher temperatures, the sensor runs faster; at lower temperatures, it is more sensitive.

The sensor is the result of pioneering research by Sandia physical chemist Bob Hughes. A team of Sandia engineers, including McWhorter and project leader Jose Rodriguez, developed the concept into a marketable product. Sandia is currently negotiating with a private company to market the system. 团

\section{For more information, call}

Paul McWhorter, Org. 1.325, (505) 844-4683.

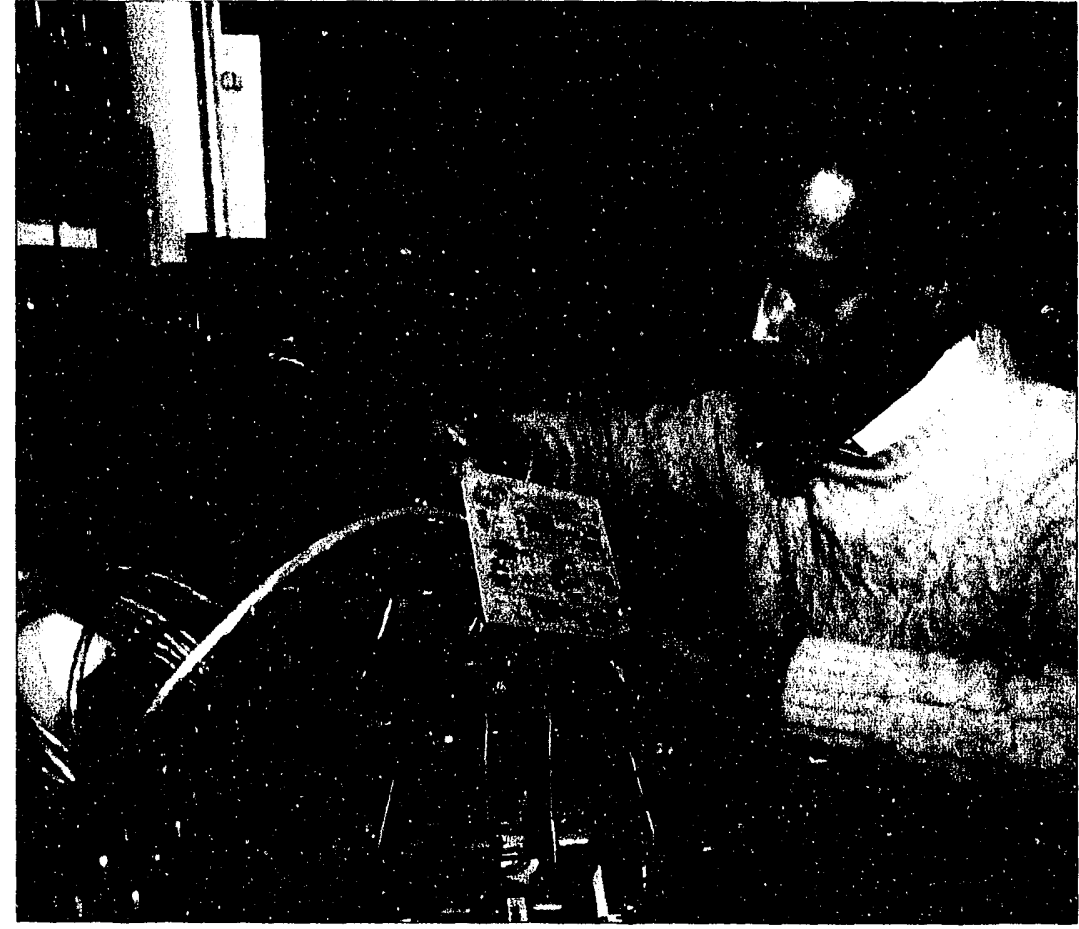

Project leader lose Rodriguez prepares a multi-point, wide-range hydrogen leak detector for delivery to NASA's Stennis Space Center. The microelectronic sensor can detect hydrogen over a broad range of concentrations, from 1 part per million to 100 percent. 


\section{Failure analysis}

\section{Manufacturers save time and money with new technique that rapidly locates microelectronic defects}

Tmagine driving from Los Angeles to New York and looking through a hole in the floorboard for a crack in the road that is one-eighth of an inch wide. That's what Sandia engineer Edward Cole likens to finding an open conductor defect in an integrated circuit.

But a new technique developed at Sandia is already helping semiconductor manufacturers to identify this defect. Called Charge-Induced Voltage Alteration (CIVA), the new technique makes it possible to quickly locate open metal conductors - minute cracks in the circuitry that can block transmission of electronic signals and cause microelectronic systems to fail. Because they are on the order of one-billionth of a meter in size, such cracks are difficult to find.

Companies that have already worked with Sandia to apply the technology to their operations include Signetics, National Semiconductor, and LSI Logic.
"LSI Logic reports that by using CIVA, the company has reduced the time needed to locate an open conductor from three weeks to less than a day in many instances," says Richard Anderson of Sandia's Failure Analysis Department, who developed the technique with Cole.

Originally developed for the Department of Energy for use in weapon systems, CIVA is a selective imaging technique that uses a scanning electron microscope to locate open conductors. Microelectronics research is one of Sandia's primary areas of concentration and is backed with funding from DOE.

In performance tests at Sandia, CIVA took only two hours to find the cause of an observed microprocessor failure that had stumped

Sandia researchers Edward Cole Jr. (seated) and Richard Anderson monitor voltage fluctuations as an electron beam is scanned over a microchip surface. The two researchers developed a new procedure, called Charge-Induced Voltage Alteration (CIVA), that makes it possible to pinpuint open metal conductors, a defect that often impairs integrated circuits.

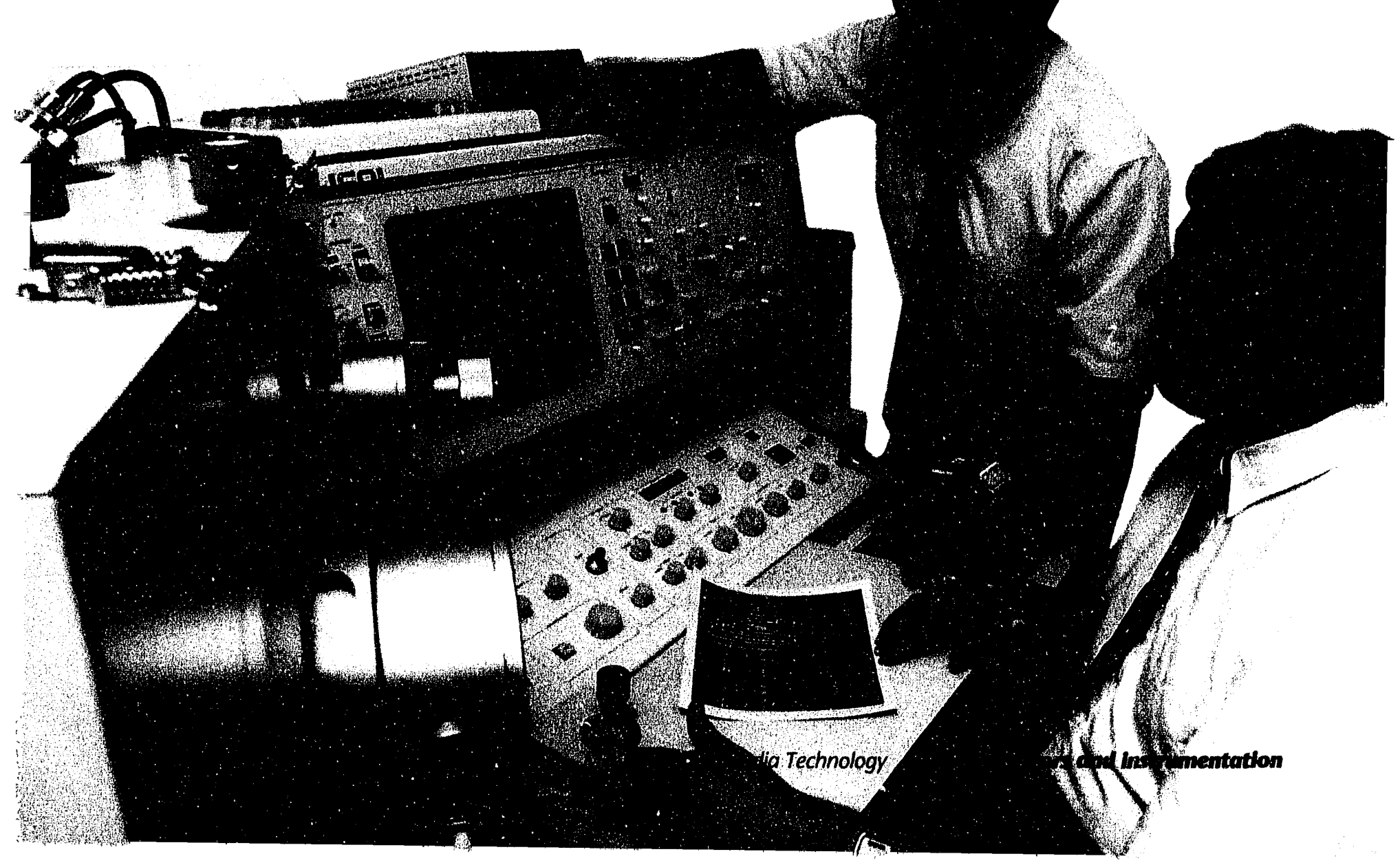


researchers for four weeks using conventional electron beam and production test equipment. The defect turned out to be a partially functioning open conductor, which was even more difficult to find than a completely nonfunctioning open conductor.

\section{Versatile and economical}

In addition to identifying weakly driven conductors, such as the partially functioning open conductor mentioned above, CIVA can be used to pinpoint defects that are buried beneath layers of metal - a helpful capability given the fact that microelectronic devices typically have several layers of circuitry.

"The technique thus makes it possible to easily and economically pinpoint defects that previously could be found only with great difficulty or not at all," notes Cole.

To locate a defect, CIVA passes an electron beam over a microchip surface and creates images based on fluctuations in the constant-current power supply. The energy of the electron beam is increased until it reaches the conductors of interest in an integrated circuit. Variations in contrast occur when the electron beam encounters an electrically open part of the conductor due to fluctuations in the voltage of the constant current source that powers the integrated circuit.

"Since open conductors are often encountered in integrated circuit manufacturing, CIVA fills an important need of industry and enhances US competitiveness," notes Anderson.

Although CIVA does require a scanning electron microscope - a piece of equipment that is typically on hand in microelectronics laboratories - it is more cost-effective than conventional circuit diagnostic procedures. The price of the constant-current source for CIVA is about $\$ 5,000$, and the amplification equipment costs about $\$ 2,000$. Conventional test equipment often costs $\$ 200,000$ or more. 团

For more information, call

Richard Anderson, Org. 2275, (505) 844-3274, or Edward Cole, Org. 2275, (505) 844-1421. 


\section{Climate studies}

\section{Sandia scientists use lasers to probe atmospheric water vapor}

$\mathbf{M}$ uch of the information people gather about the world is derived from light from what they can see with the naked eye. Human vision is both passive and remotepassive in the sense that the viewer does not generate the light that produces the information, and remote in the sense that the viewer is physically removed from the object being observed.

With proper scientific instrumentation, this capability, known as "passive remote sensing," can be changed to "active remote sensing." An active system generates its own outgoing signal and then detects some measure of the signal that is altered by objects in its path. Examples of such systems are radar (well-known, among other things, as a technique for tracking aircraft), and lidar, which stands for Light Detection And Ranging, the optical analog of radar. Radar uses radio signals, lidar uses light signals.

A lidar system sends out a laser pulse and uses a small- to medium-size telescope to detect the fraction of the light that is backscattered toward the viewer. Lidar systems can observe not only "hard targets," ranging in size from vehicles to microscopic aerosols, but can identify trace chemical species in the atmosphere using spectroscopic techniques. Lidar systems are valuable for many studies of critical national interest, such as global climate change, hazardous chemical waste detection, and arms control and treaty verification.

Sandia is contributing to the Department of Energy's remote sensing capabilities through

the development of a mobile Lidar Testbed that enables researchers to study the atmosphere in the ficld. The instrumentation is housed in two trailers - one trailer serves as a "lab on wheels" and carries the laser, telescope, detector package, and related equipment; the other trailer provides data analysis and support utilities. The system is currently being used in DOE's Atmospheric Radiation Measurement Program, established to investigate the role of clouds and atmospheric moisture in trapping radiative heat, a key area of concern related to global warming.
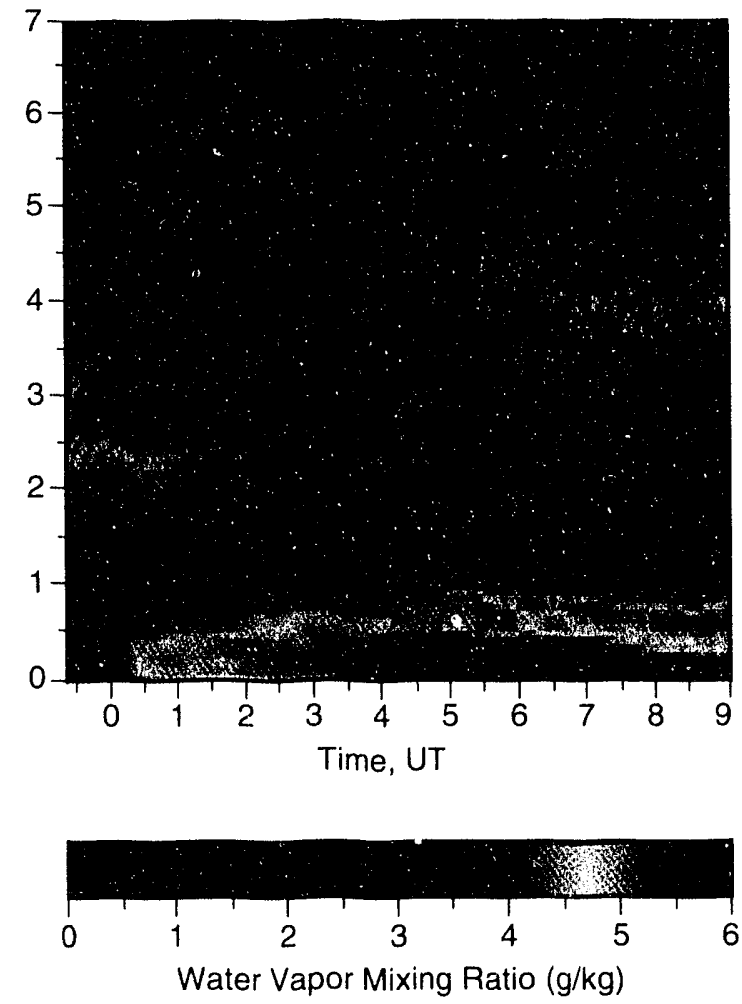

The Sandia Lidar Testbed measures the moisture content of the air (expressed as grams of water per kilogram of dry air). This false-color image shows the water vapor mixing ratio measured during a 9 1/2-hour period at the NASA Goddard Space flight Center the night of Nov. 11, 1992. (UT is universal time, the same as Greenwich Mean Time.)
Profiles of chemicals, aerosols, and clouds

Sandia scientists are currently using the Lidar Testbed configured as a Raman lidar for profiling atmospheric water vapor, a vital ingredient in global climate change as the most abundant radiation-absorbing gas. A Raman lidar system sends out a laser pulse and, by timing the backscattered return signals, is able to determine water-vapor density as a function of distance from the lidar. The return signals also can be used to profile cloud heights, aerosols, the boundary layer of the earth's atmosphere, and other molecular species by using the appropriate wavelength of light for each desired constituent. Sandia has teamed with researchers at NASA's Goddard Space Flight Center to develop the next generation of Raman lidar probes for both daytime and nighttime profiling of atmospheric water vapor.

"The potential warming effect from clouds and atmospheric water vapor far exceeds that of carbon dioxide, often regarded as the most significant contributor to global warming," says John Goldsmith of Sandia's Diagnostics Research 
Department. "V vater vapor is a significant absorber of infrared radiation or heat."

"The effects of clouds and water vapor may br the most important factors for predicting possible climate change as a rt.ponse to human activity," adds fellow researcher Marshall Lapp of the Global Climate Change and Rernote Sensing Office.

By looking directly upward and recording repeated vertical profiles over an extended period of time, the lidar system can produce an image of the water-vapor content of the atmos. phere as it passes overhead. Although nighttime observations are often superior because of the absence of significant background light that interferes $\mathrm{wi}^{\text {th }}$ the backscattered signals, the focus of the Sandia-NASA resedrch is to develop instruments for making daytime measurements of water vapor that are readily available for use in the field and are not overwhelmed by bright sunlight. Climate modelers need to include daytime observations in order to adequarely characterize the earth's radiation.

Sandia's Lidar Testbed can accommodate a variety of lidar techniques to do atmospheric characterizations. The Raman lidar configuration is focused on detecting atmospheric species at relatively high concentrations. Other techniques, such as fluorescence and DIAL (DIfferential Absorption Lidar), are more sensitive to specific chemical species but require greater experimental complexity. Sandia is developing such additional instrumentation to make the testbed useful for an expanded range of DOE investigations in the areas of defense, environmental monitoring, and energy.

For more information, call

John Goldsmith, Org. 8351, (510) 294-2432, or

Marshall Lapp, Org. 8102, (510) 294-2435.

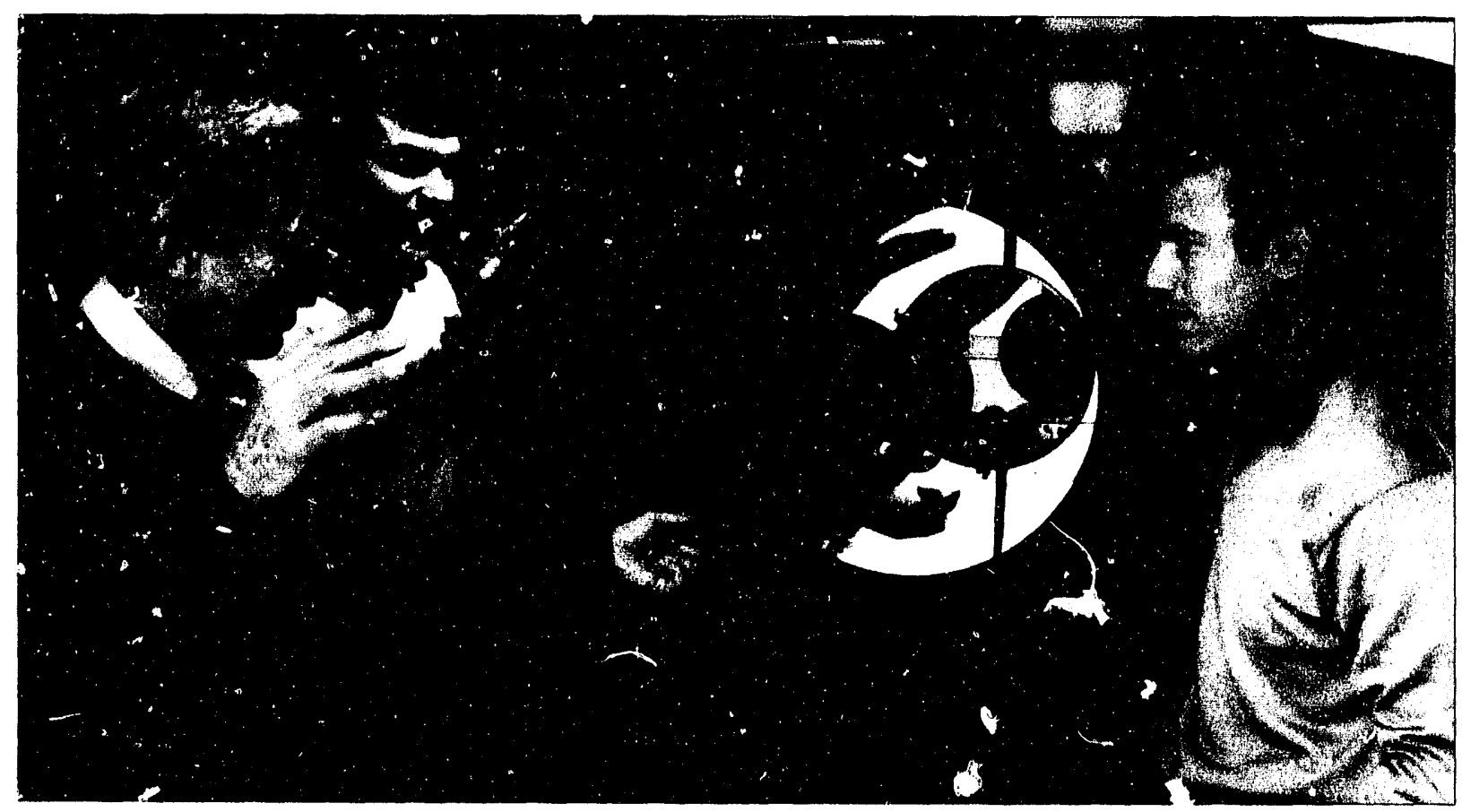

Researchers (from left) Marshall Lapp, Scott Bisson, and John Goldsmith discuss atmospheric moisture studies done with the Raman lidar telescope, a component of a remote sensing device that gathers information about water vapor in the Earth's atmosphere. 


\section{Laser weapon sensors}

\section{Laser detection technology could benefit treaty monitoring}

For years, concerned scientists have talked of Flir iting ground-based, anti-satellite laser weapon systems. Currently, a significant number of laser systems potentially have sufficient power to cause satellite damage ranging from disturbance of optical sensors to complete burnthrough of parts. Presently no physical systems exist to monitor or verify deliberate or inadvertent lasing of satellites.

In February 1990, the Federation of American Scientists (FAS) suggested to Congress that such lasers could be iletected by monitoring off-axis aerosol scatter. Off-axis refers to the light that is scattered by particles (aerosols) in the atmosphere not directly aligned with the laser beam.
This phenomenon is what makes searchlights visible over great distances, though lasers do not scatter as much light.

Using smoke as the scattering medium, FAS researchers demonstrated the detection concept with a simple sensor. Although the experiment did not prove the large-scale feasibility of the concept, it did indicate that such detection could be part of a system to monitor atmospheric testing of high-energy lasers.

Before negotiating a treaty involving a monitoring system, it is necessary to identify measurable parameters and negotiate permissible thresholds. This in turn requires accurate measurement of these parameters. For example, satellite damage levels can be directly correlated to the brightness of a laser. However, brightness cannot be measured directly; it must be inferred from other measurable laser parameters.

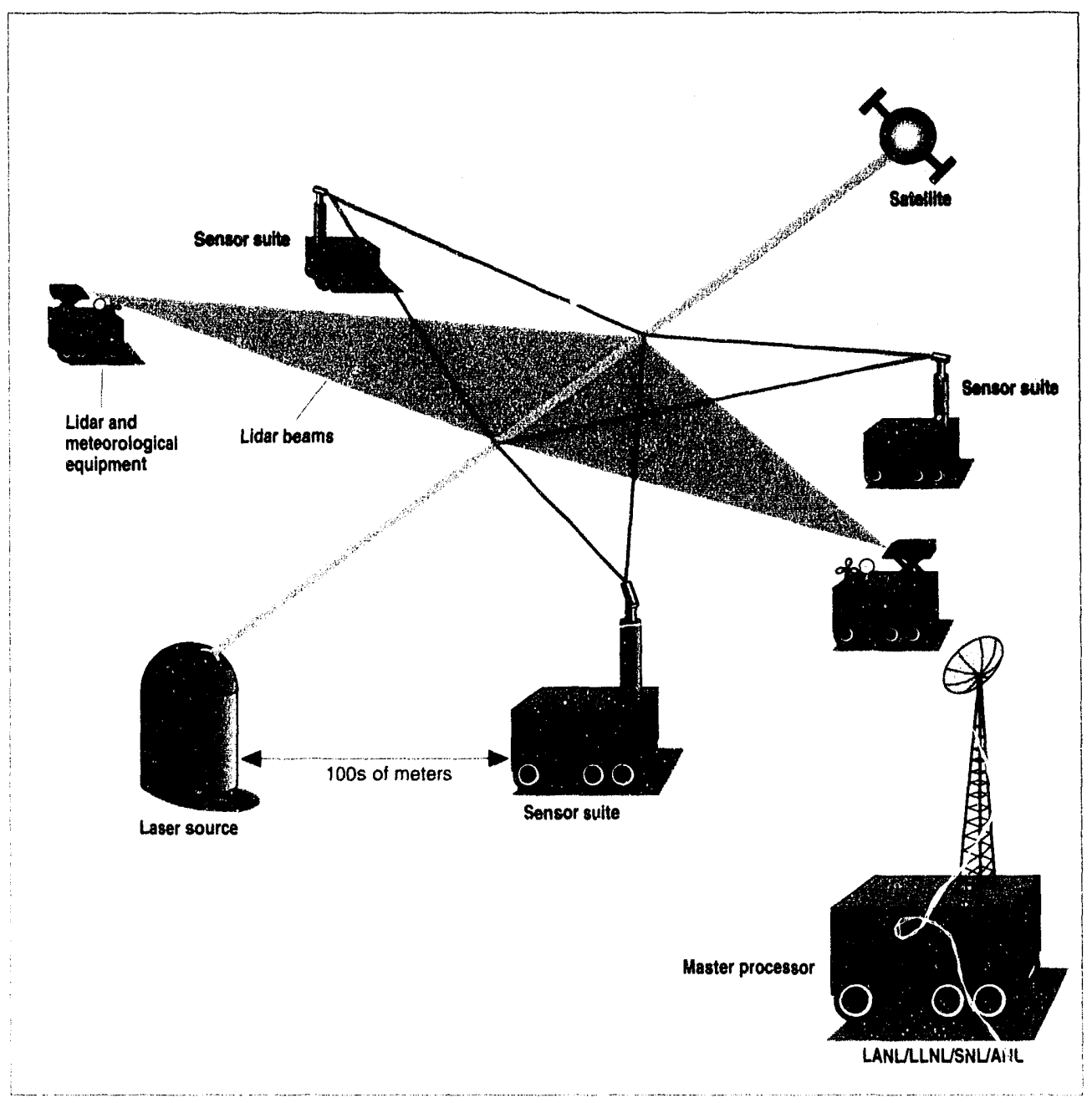

Suggested layout of high-energy laser monitoring systern shows sensors that collect scattered laser light and meteorological equipment that characterizes the atmosphere. The master processor collects, analyzes, and stores data for archiving or transmitting to other locations. 


\section{DARPA-funded research}

In 1990 Congress directed the Defense Advanced Research Projects Agency (DARPA), under the technical guidance of a panel of researchers and professors called the Jason committee, to study radiometric detection of off-axis laser beam aerosol scatter. DARPA established programs at Sandia, Los Alamos, Lawrence Livermore, and Argonne national laboratories to use existing equipment and some domestic lasers to verify the feasibility of the technique.

During this testing, researchers validated that the most problematic characteristic was variability of signal strength at the detector. Depending on the time of day, weather conditions, and the angle to the laser path, the strength of the signal arriving at a fixed site could vary by several orders of magnitude.

Based on this work, researchers at Sandia and the other national laboratories are now developing preliminary technology for a monitoring system to detect and characterize high-energy lasers firing into the atmosphere. The system will calculate the power, wavelength, and propagation direction of the laser, and by correlating that information with the beam diameter, it will infer the laser's brightness. Ultimately, this preliminary system will help establish the accuracy with which these laser parameters can be measured, and will begin to define the requirements for a deployable system.

The system will consist of a suite of optical detectors operating at wavelengths between 1.0 and 1.5 micrometors. The initial goal is to detect and characterize a medium-power laser at distances up to $i$ kilometer. The modular design of the sensors wi!l permit substitution of different detector materials and allow the system to be tailored to monitor different laser wavelengths. Sandia and Lawrence Livermore are independently developing remote sensors for this task. Sandia, Argonne, and Los Alamos are developing meteorological equipment for measuring and characterizing the scattering properties of the atmosphere.

The remote sensors and meteorological equipment vill collect and send data to a master processor that will log, integrate, and validate the data. An inversion processor will factor in the many atmospheric and testing variables to calculate the characteristics of the laser. Sandia's responsibilities include the design of the computer network, the inversion processor and its algorithms, and integration of all equipment into the system.

\section{Project milestones}

Using satellite technology, Sandia has developed an optical sensor capable of measuring average incident irradiance, signal wavelength, and a twodimensional angle of departure. The instrument incorporates a linear array sensor and a customdesigned diffraction grating. The detector material is sensitive to near-infrared wavelengths without the need for cryogenic (subzero) cooling. Field tests have demonstrated the sensor's ability to detect and characterize a medium-energy laser from a distance of 300 meters while looking into a bright, sunlit sky. "We anticipate that signal processing will enable the sensor to detect the laser from even greater distances," says Optoelectronic Design Department Manager Anthony Medina.

Sandia's development of the data integration computer network, the inversion processor, and the metcorological instrumentation is in the final stages. The four national laboratories will demonstrate the system to DARPA this spring, when Sandia scientists will fire a pulsed laser under varying conditions of weather, daylight, beam propagation geometry, and distance. The results of this experiment will identify baseline requirements for a potentially deployable system. 田

For more information, call

Lydia Boye, Org. 5902, (505) 844-1708, or

Anthony Medina, Org, 9222, (505) 845-8120. 


\section{Human-machine interaction}

\section{People vs. too much data: advanced computer systems assist decision-making}

$S_{\mathrm{L}}^{\mathrm{c}}$ cene from an operating room of tomorrow: Laser in hand, a surgeon prepares to operate on a brain tumor. A nearby computer monitors vital signs such as blood pressure, pulse, respiratory rate, temperature, intercranial pressure, and any reactions that could result from anesthesia combined with other medications the patient is taking. If a particular condition, such as heartbeat, becomes critical, the computer sounds an alarm and the information appears on the computer screen as a pictorial display rather than a complicated digital reading.

As the surgeon begins to probe the brain in search of cancerous tissue, a three-dimensional display on the screen portrays the actual tumor, allowing the doctor to precisely direct the surgical probe.

Though it may sound futuristic, this is only one example of how state-of-the-art computer systems - an area of demonstrated technical leadership at Sandia - will one day enable humans presented with a deluge of data to make more accurate decisions more quickly.

\section{Wide array of applications}

Similar systems cooperatively designed by Annette Sobel, medical doctor and a human factors engineering specialist in Sandia's Medical Department, are already in use on board Air Force reconnaissance systems and commercial aircraft. While working for the Air Force, she helped develop a new concept of pictorial displays in aircraft cockpits that are now also being used in the Boeing 757 and potentially in hypersonic vehicles.

A former flight surgeon for the Air Force and the National Aeronautics and Space Administration, Sobel is developing human-machine interface systems for Sandia's Exploratory Systems Department with funding from the Department of Energy. Such systems improve human performance in processes that can't be fully automated, from environmental monitoring to biomedicine. The goal is to eliminate the need to sift through innumerable bits of data.

"Such systems seek to improve two critical human performance criteria: incidence of error and reaction time," says Sobel. "For example, 80 percent of aircraft accidents are due to human error, because there's too much information presented at once, and in such a format that everything is given equal emphasis."

Of course, in order to get such systems to work, it is necessary to program the computer in advance to rank the data by priority and present only that information that is critical. In the military, similar systems are known as Automatic Target Recognizers (ATRs). An ATR employs a computer that constantly scans for points of interest, such as nuclear reactors, SCUD missiles, specific kinds of weapons, or camouflaged sites, and assigns a probability index to each one. This probability index is based on a decision algorithm that scans as many as 10,000 potential images. The computer can also enhance targets as needed by changing the viewing angle, the resolution, or other parameters.

In the doctor's office, such systems could lower health care costs. For example, instead of studying a multitude of raw data from CAT scan images, a radiologist would view only a portion of the data selected by the computer and presented as a three-dimensional image. A probability index, designed to rule out false alarms caused, for instance, by patient movement or fatty tissue, would accompany the display. The radiologist then would compare this image to known tumor images.

"In diagnosing a brain tumor," notes Sobel, "you could have 50 to i00 digitized images stored in the system that neurosurgeons normally rely on viewing manually. No system currently exists to pull up only the most critical images at different angles and resolutions and present them in a three-dimensional image. So a doctor chooses them based on training, which takes longer and costs more. Radiologist fees are based in large part on how long it takes to scan the images."

In the workplace, human-machine interface systems could be used to monitor multiple medical conditions related to diet, sodium levels, hypertension, diabetes, or major surgery. They could be used at home, permitting patients to monitor themselves on a daily basis, reducing the number of trips to the doctor. Such systems also have great potential, notes Sobel, to eliminate "polypharmacy" - the administration of overlapping and sometimes incompatible drugs by different doctors at different times. 
In environmental monitoring and industrial hygiene, human-machine interface computers can be used to monitor beryllium exposure in manufacturing, contaminants in soil, or the cooling processes in a nuclear reactor. They could be programmed to recognize how much argon gas is tolerable in a drilling operation and sound an alarm if argon levels exceed that amount. As in other applications, the computer would draw attention to those items that have reached a critical threshold.

Other potential uses include chemical decontamination, diving operations, or anything done in a confined space. NASA is even developing a three-dimensional, all-aspect viewing angle display to be incorporated inside astronauts' helmets when they are tethered outside a spacecraft. The display would provide both astronauts and Earth observers with real-time information, eliminating much of the guesswork involved when astronauts attempt to capture and rescue a damaged satellite. 团

For more information, call

Annette Sobel, Org. 7030, (505) 845-8157.

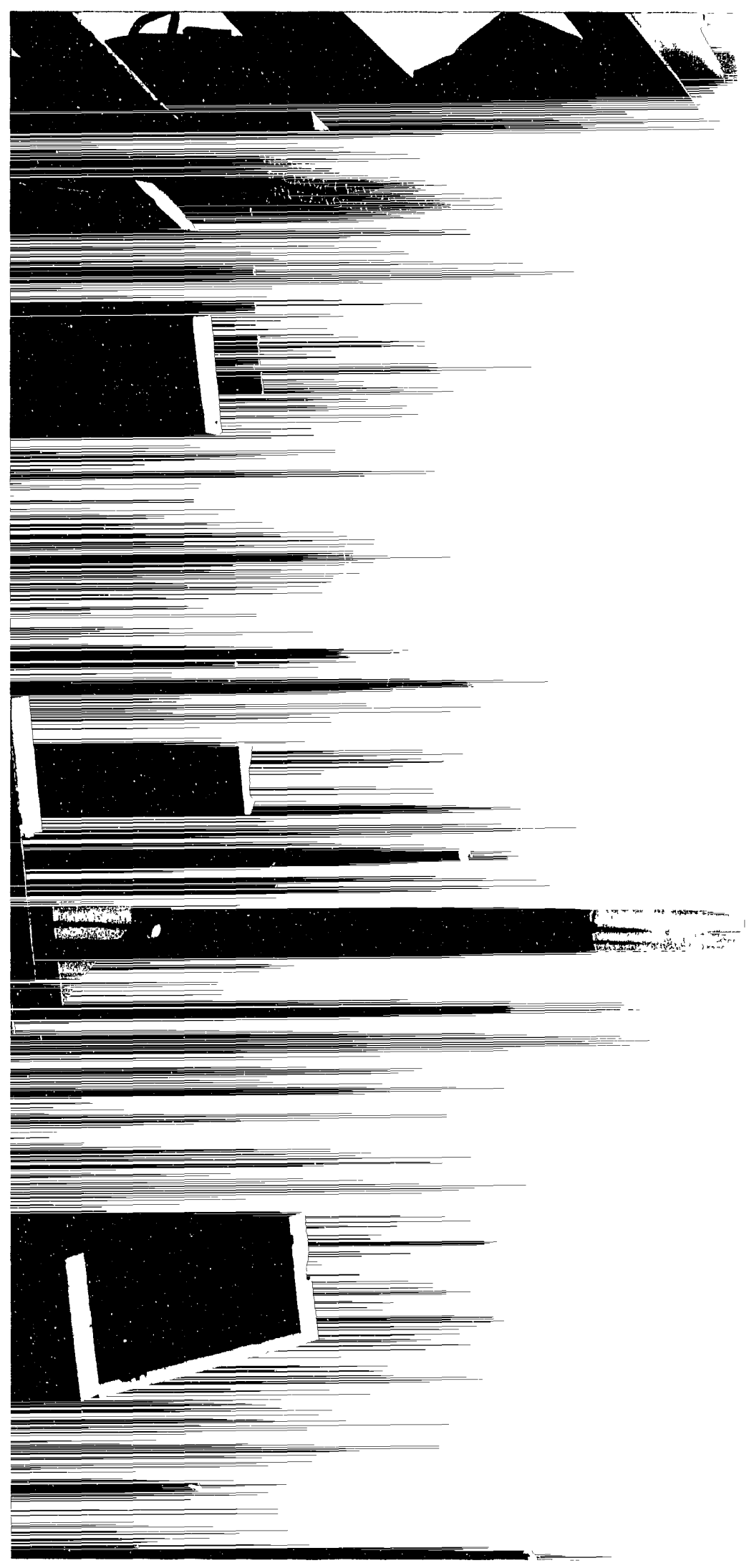

As a nonrated pilot trainee/flight surgeon in the Air National Guard, Annette Sobel prepares to fly an F-16 in January 1992. 


\section{Communications system \\ helps DOE monitor trailers carrying sensitive goods}

Comewhere in the mountains above AlbuSquerque, New Mexico, a van travels around on local roads near a ski resort and a tramway that stretches from the desert floor to the top of the mountain. Every few minutes or so, the driver of the van sends a message to a control room at Sandia National Laboratories.

An operator in the control room zooms in on a computer map until he finds - there it is Sandia Peak, and a small vehicle icon flashing on the screen. The icon marks the spot on the Sandia Crest Highway where the driver's message originated. The map on the screen is so detailed, it even shows foot trails.

"There's snow up here," says a message from the driver, relayed from a spot 4,000 feet higher in elevation than the city limits. Actually, this is an interesting bit of weather information, as it happens to be the first snow of the year. The operator checks the condition of the van and notes that all is well.

The scenario is reminiscent of a video game, where a human at a control panel can manipulate multiple imaginary vehicles all at once, continuously monitoring their condition and whereabouts. Except in this case, the vehicles are real. And they can be monitored on multiple screens displaying geographic data from all over the country.

This system is known as STARBASE, which stands for Security Tracking and Response Base. A user-friendly operator display system, STARBASE was built for the Department of Energy's Transportation Safeguards Division to track the location and condition of government-owned trucks and trailers hauling nuclear materials. STARBASE is the latest in a series of such systems, which Sandia began designing for DOE in the 1970s.

\section{New capabilities and upgrades}

With each successive version, new technology makes it possible to upgrade and expand the capabilities of the system. The new system installed in 1992 has more accurate and detailed maps than previous systems, as well as improved tracking ability. It can display and trace a vehicle's path through several states and communicate via satellite or over the air.

"We've built a lot of redundancy into the system," says project leader Jayne Williams. "The idea is that if one system fails, we have a backup system. To enhance reliability, we have alternate paths for sending and receiving information."

STARBASE does a great deal more than track the location of sensitive vehicles. It also monitors their overall condition - sensors, battery voltages, alarms, door locks, and other critical items. It looks for anything abnormal, such as a lack of response from the driver or a sign that someone is trying to break into a cargo area. If something goes awry, the system has the ability to initiate emergency procedures. The computer prioritizes and displays emergency information to the operator, who can manually call up more data as needed. For example, a simple touch of a finger calls up critical support facilities, such as the nearest airport or hospital. The computer can even speak to the operator, providing timely instructions and warnings much the way a car alarm reminds a driver to fasten a seatbelt.

The display of geographic information on STARBASE is extremely versatile. An operator may request as little or as much detail as desired. For example, by touching the appropriate place on a screen, the user may choose to view all the roads in an area, including residential streets, or eliminate clutter and show only state and interstate highways.

The reason for such a user-friendly system is to improve human performance in an emergency. "We've learned during tests that in a crisis, when the adrenalin flows, people tend to make mistakes," says Bob Cover, chief designer of STARBASE who has helped design secure communications systems for DOE since 1973. "Many geographic information systems are unacceptably difficult to use, especially in the environments under which we have to operate."

Sandia has a small fleet of vans that researchers track for practice. Each van is equipped with a computer keyboard that the driver uses for transmitting written messages, as well as radios for voice communications. In the interest of security, of course, information transmitted between the control center and the vehicle is encrypted.

Most of the equipment used in STARBASE is off-the-sheif technology. The vehicle location 
system is similar to a monitoring system used by the trucking industry. The communication system designed by Sandia incorporates encryption and other security features.

STARBASE has many potential applications. It could be used to monitor nuclear reactor sites, individual buildings, or vehicles in smart highway applications. It has been adapted to help manage DOE's Emergency Operations Center in Washington, D.C.

Future upgrades to STARBASE may include three-dimensional surtace information as part of the mapping data and landmarks and mile markers to help drivers describe their location to the control center. 团

For more information, call

Jayne Williams, Org. 9617, (505) 845-8709, or

Steve Weissman, Org. 9617, (505) 844-4570.

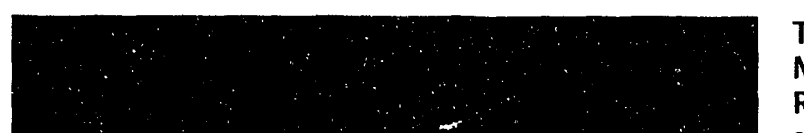

The STARBASE computer map of Albuquerque, New Mexico (left), shows several levels of detail, such as the Rio Grande, the airport on Kirtland Air Force Base, interstate highways, major thoroughfares, and local streets.

A closeup of a portion of the city (below) shows the airport, $1-40$, and local streets and thoroughfares.

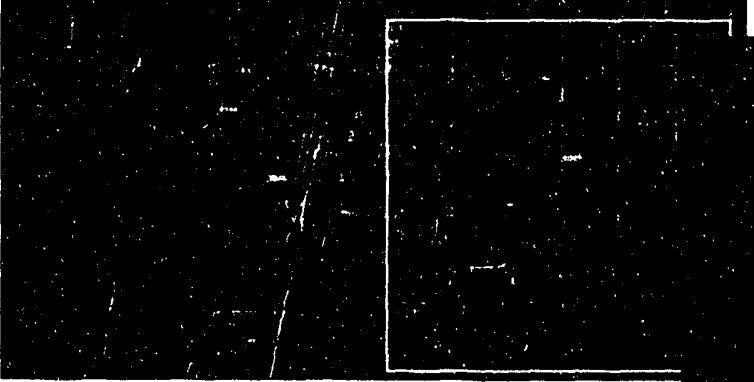




\section{Receiver decoder}

\section{Subsystem helps secure vehicles hauling sensitive cargo}

$\mathrm{A}$ Ithough its contents and destination is held under wraps, a vehicle carrying sensitive cargo for the Department of Energy has extensive security systems to keep its contents safe from intruders. From specially trained employees to state-of-the-art communications, such vehicles are equipped with many systems for preventing unauthorized access.

Sandia has been designing transport vehicles for DOE for years. One of the newest devices developed for these vehicles is a receiver decoder that replaces an older system developed for use in Vietnam. Originally used to detect objects on the ground and relay the information to a control center, the older system was repackaged in the early 1970 s as part of the vehicle security system.

The new receiver decoder, designed by Sandia engineers Tim McCorkendale and Don Small, has been greatly improved and has several advantages over the 1970 s version. Like other modern electronic products, it is much smaller than older devices and packs more components onto a single printed circuit board. It has fewer parts, making it easier to produce and maintain, especially in light of the fact that it was becoming harder and harder to find replacement parts for the older system. The new receiver decoder has a single board of microelectronic circuitry; the old security system had three. Moreover, the old system had to be calibrated about once a year, while the new system can continuously inspect itself for problems. All of its time-dependent processors are linked to a state-of-the-art, highly precise, microprocessor clock.

"We conducted many tests of the system to make sure it would function under any potential conditions," says McCorkendale. "It's essential that the ruggedized design of the components performs reliably during the entire life cycle of the system."

"Just like the car you drive, the parts have to be tested to make sure they work in a variety of environments," adds Small. "And like automobile manufacturers, we test the system by subjecting it to conditions that are actually worse than would be encountered in reality."

\section{Delivered ahead of schedule}

Small and McCorkendale got the assignment for designing the new system as a result of a jogging workout one morning with several other Sandians. One group of staff members mentioned the task; another group offered its expertise. The end result was a prototype that was delivered several months ahead of schedule and subsequently fielded in 1992. In fact, just three weeks after Sandia's Electronic Components Center and Transportation Systems Center got together to negotiate requirements, the two researchers produced a compact, ruggedized printed circuit board design. This is when they replaced the three boards of the older system with one board. Their design work was facilitated by using networked Computer-Aided Engineering workstations, a technology known as concurrent engineering.

To provide DOE with a product that would meet system requirements as inexpensively as possible, the researchers

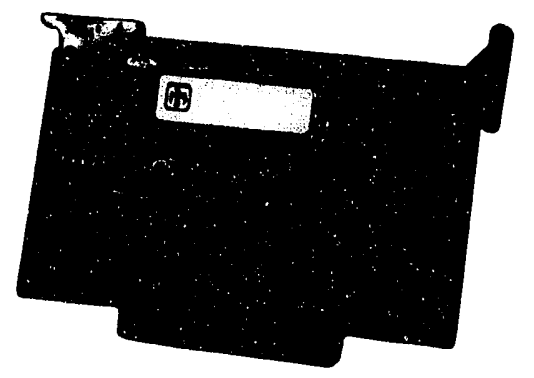

The security system is much smaller than older devices and has a single board of microelectronic circuitry rather than three.

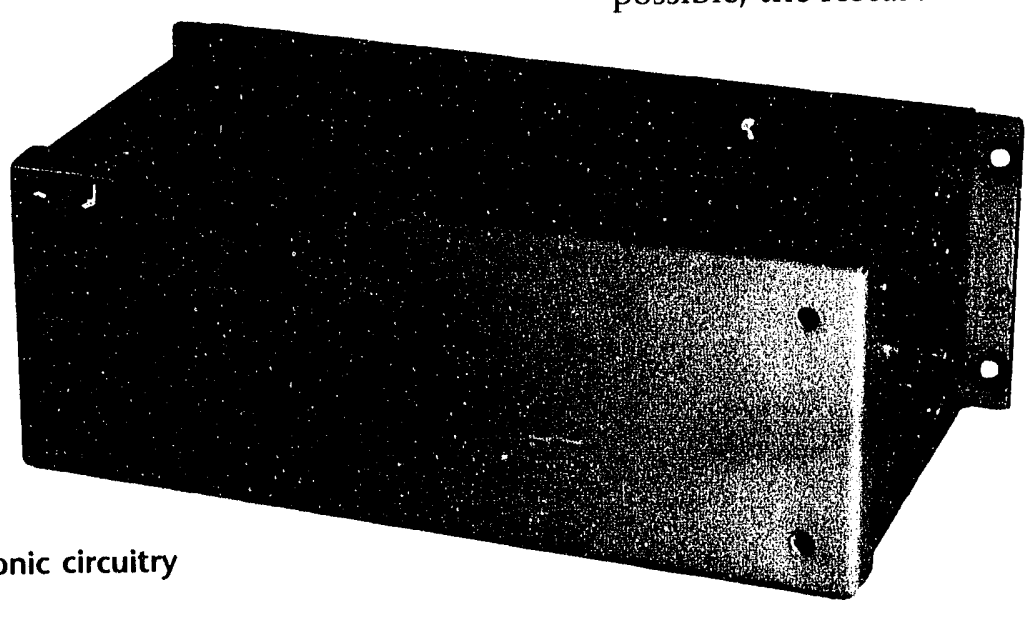

Information management 
studied the performance specifications for both military and commercial components and combined components from both areas. The final production cost of the printed wiring assembly is only $\$ 300$. To ensure that the system would meet performance standards, they conducted humidity, vibration, shock, and temperature tests of the boards to identify the potential for so-called "infant mortality" - that is, early failure of components - to prove that the system would work even with some individual components that did not meet the more stringent military specifications. All of the boards were tested this way. When the final design was ready, an outside company built the boards, and

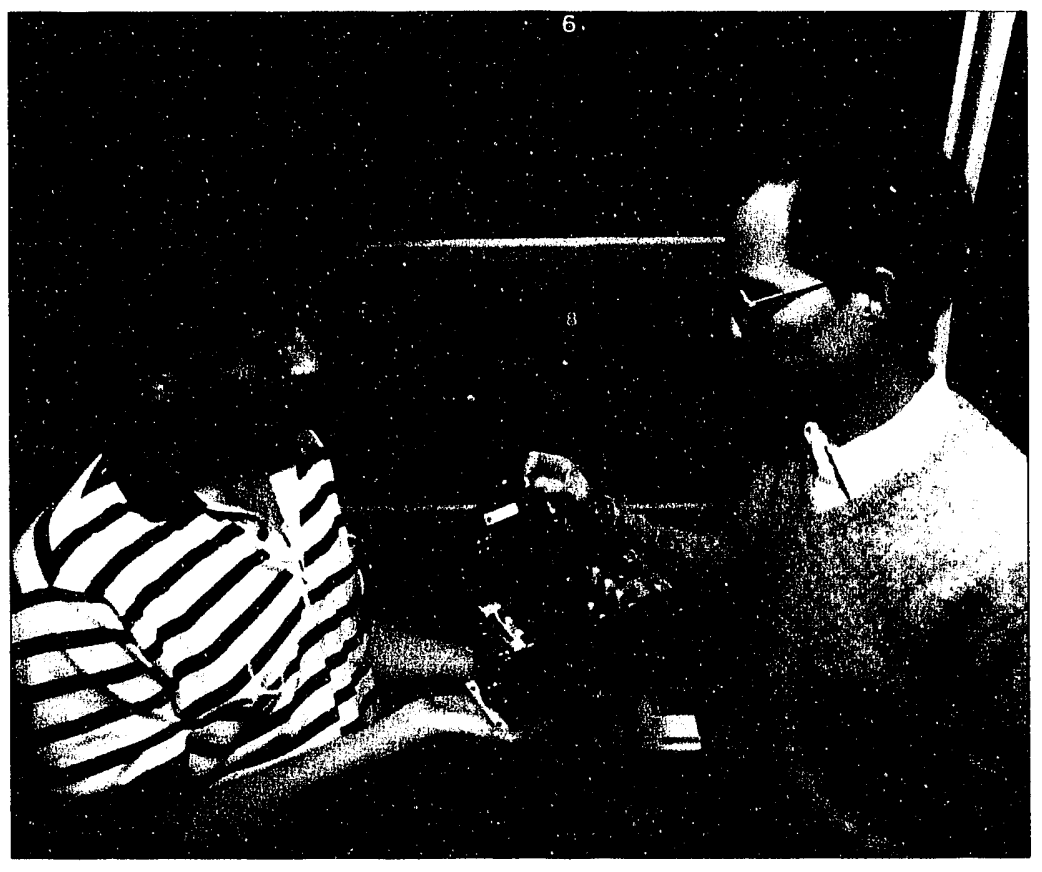
another company installed the circuitry.

To get as many fresh ideas as possible, the two designers held review sessions with other Sandians. Among the more interesting features of the new receiver decoder is its self-diagnostic capability. If something went wrong with the older system, no one would know it until the next maintenance check. The new system's diagnostics track failure trends, looking for anything out of the ordinary in the microprocessor, the power source, or the encoding process. If something unusual occurs, the system automatically notifies the user that maintenance is required. 团

Engineers Tim McCorkendale and Don Small examine the components of a receiver decoder that helps secure transport vehicles.

For more information, call

Tim McCorkendale, Org. 2314, (505) 844-6392, or

Don Small, Org. 2314, (505) 844-9500. 


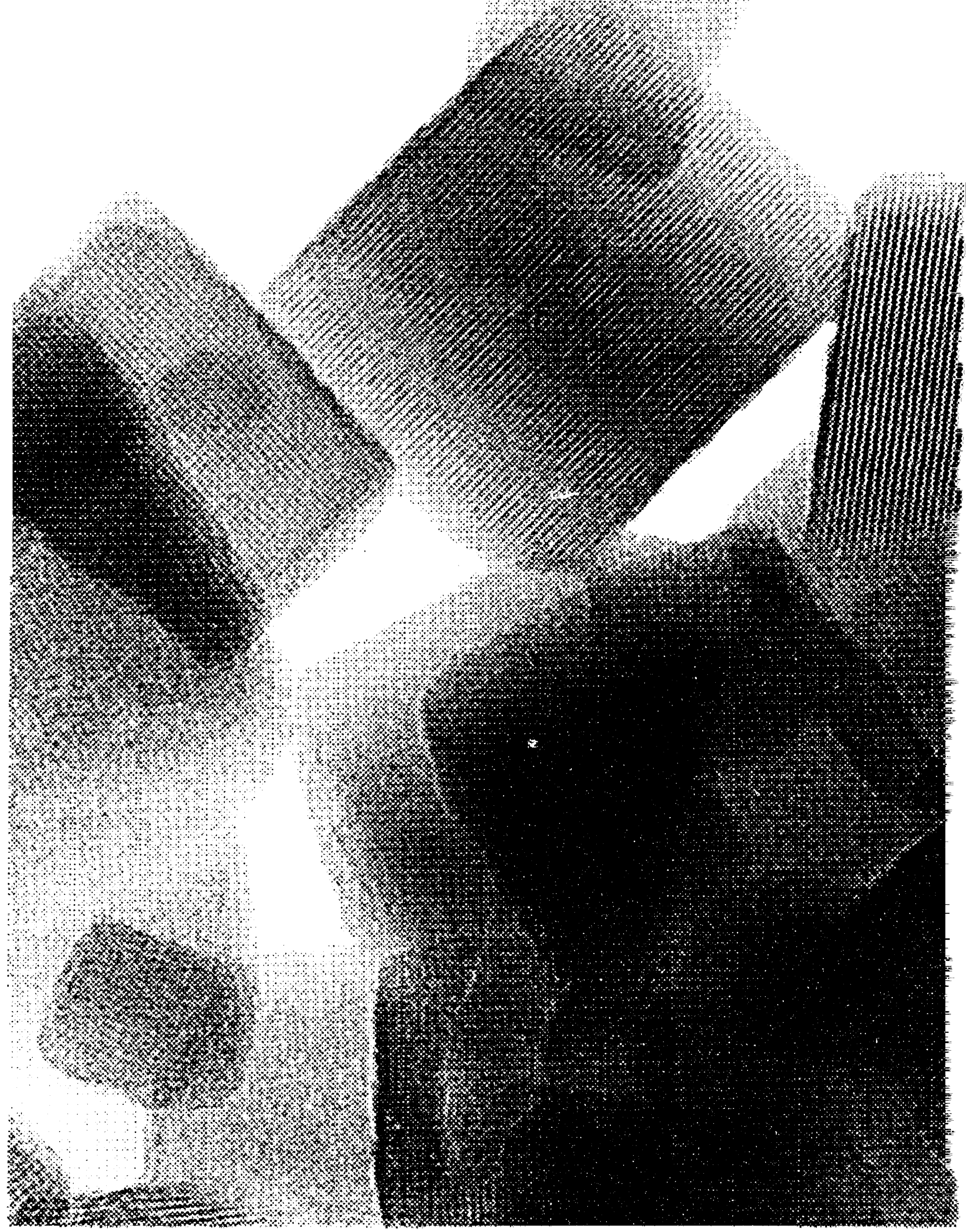




\section{Energy \& environment}

Cesium ions fit between the lattice planes in the crystalline structure of these silico-titanates. Developed at Sandia, these silico-titanate materials can be used to remove cesium from radioactive waste. 


\section{Solar thermal energy}

\author{
Sandia and Cummins Engine are \\ developing an efficient, \\ low-cost dish-Stirling system \\ that may power electric utilities
}

Tn today's increasingly environmentally orilented marketplace, solar power is no longer the oddity it was in the early 1970s. Residences and businesses both in urban and rural areas are often equipped with photovoltaic panels that directly convert sunlight into electricity.

But while it is less common, there is an emerging technology known as solar thermal power generation. This method uses the sun's heat to power an engine to generate electricity.

For the past several years, Cummins Engine Company, a leading manufacturer of diesel engines and generators, has chosen to pursue dish-Stirling solar thermal technology, in part because of its high efficiency and in part because newer materials and technology make it possible to reduce the cost of manufacturing a solar thermal power system.

In a cost-sharing venture with Sandia, Cummins is working on commercializing this technology, with the goal of reducing the cost of electricity, particularly in remote geographic areas, and providing a new product to users of Cummins diesel generators. The dish-Stirling technology uses a sun-tracking system to concentrate solar energy onto a free-piston engine that has only two moving parts and doesn't require lubricating oil.

The moving parts are pistons - a displacement and a power piston. In the Cummins engine, the pistons are free-floating and driven by pressure from heated gas. The engine has no crankshafts, no cams, and no connecting rods. Its design is different from the design of a kinematic Stirling engine, which like most engines has linkages and other moving parts, all of which have to be oiled.

Development costs are being shared equally by Cummins, including its industrial partners, and The Department of Energy through a 3 1/2-year, $\$ 14$-million program. Supported by DOE since the 1970s, dish-Stirling technology has achieved peak net efficiencies of more than 29 percent; that is, it can convert 29 percent of the sunlight that illuminates the collector to electricity.

"It's a very exciting technology," notes Sandia

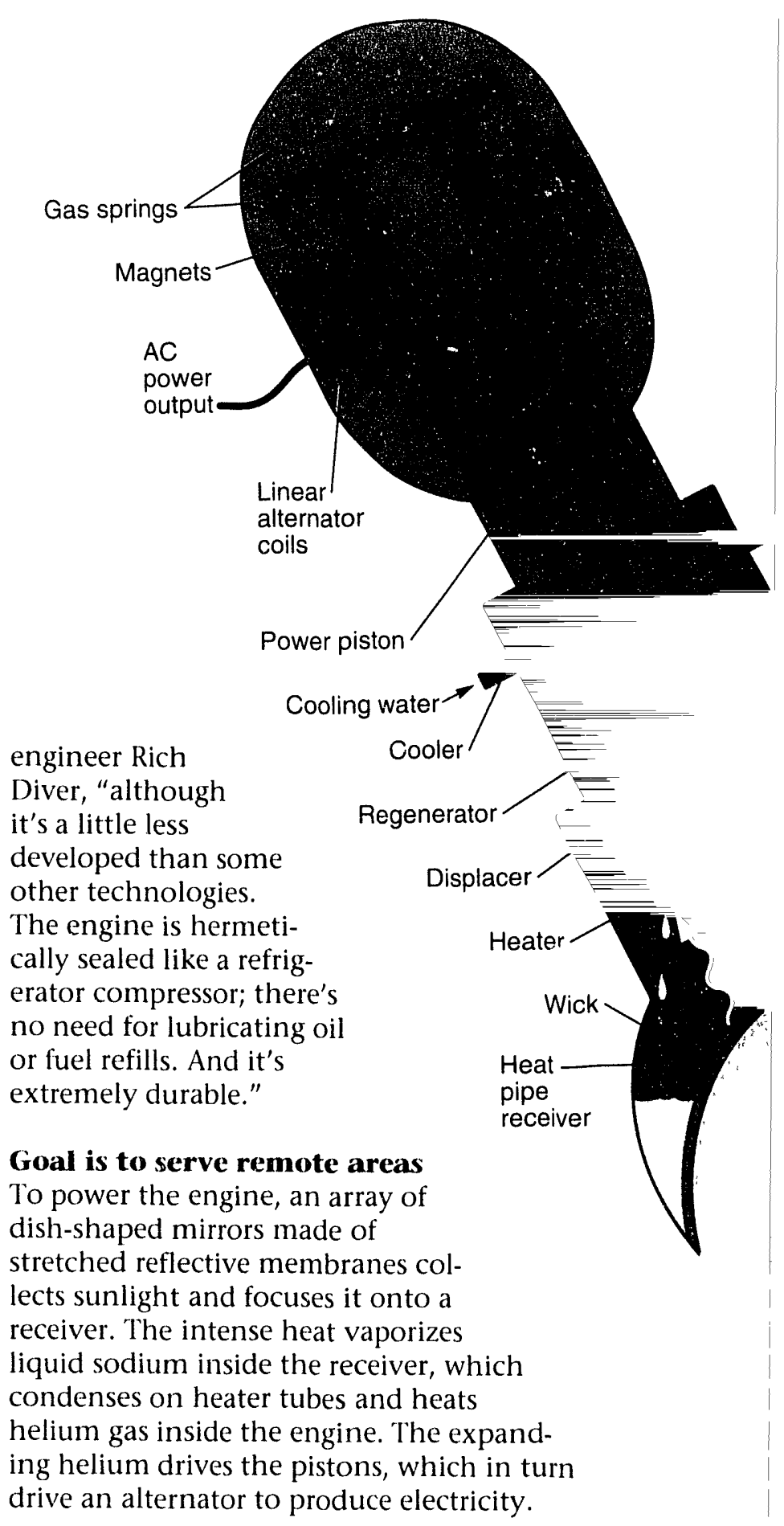

The dish-Stirling engine (above) has only two moving parts and does not require lubricating oil. Sunlight hits the receiver and evaporates the liquid sodium, which heats the helium inside the piston chamber. As the helium is alternately compressed and expanded, it drives the power piston back and forth. Magnets on the power piston pass through an electrical coil and produce an alternating current. 
Tne dish-Stirling system produces 120 -volt electricity. A single module can generate more than 5 kilowatts of power, enough to pump water or provide power to a remote village or hotel site. Individui: modules can stand alone or be connected to a grid. They can be used tu pow'er signals at railroad crossi'..gs or navigational aids to : vats offshore. And like other forms of solar energy, dish-Stirling solar thermal systems are environmentally benign.

An added advantage of the system is its versatility - when the sun doesn't shine, the engine can be heated with an auxiliary fuel such as natural gas, propane, or heating oil. The system is equipped with a microcomputer that ralchuates the sun's position and keeps the system focused on the sun.

By wising the dish-Stirling system, Cummins hopes to bring the cost of electricity down to a more competitive level in remote markets, where electricity frequently costs 30 to 60 centi per kilowatt-hr .i, compared with, say, 8 to 10 cents per kilowatt-hour in Albuquerque, New Mexico.

"If you liveci far away from a power grid, it would probably be a lot cheaper to install something like this than to pay for $\mathrm{r}$ nning an electric line to your property," notes Diver.

Currently, Sandia is soliciting cost-sharing proposals

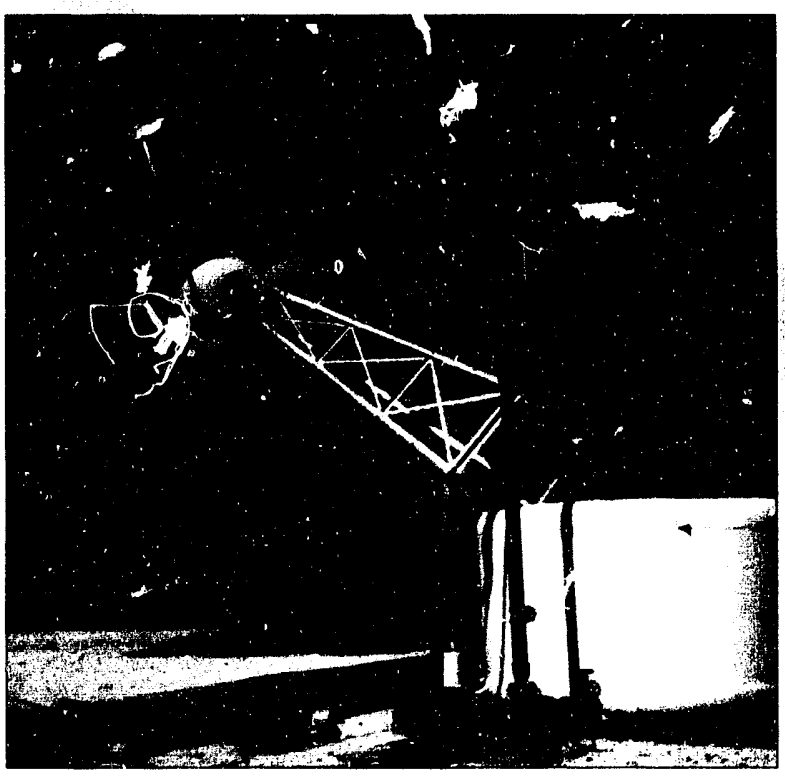

from solar-power companies to develop modular dish-Stirling systems for utility-scale applications. Such systems could provide power to communities in sparsely populated areas, such as the western United States, at more competitive rates.

"I think that working with industry through joint ventures and cost.sharing arrangements is the wave of the future," says Diver. "It's a strategy for teaming national laboratories with private companies." 团

For more information, call

Rich Diver, Org. 6216, (505) 844-0195.
A Cummins Engine Co. dish-Stirling system in Abliene, Texas (left), converts the sun's energy to electricity to pump water in rural locations. The system uses sodium to transfer heat from sunlight to the engine; the sunlight is collected by an array of stretchedmembrane mirrors. 


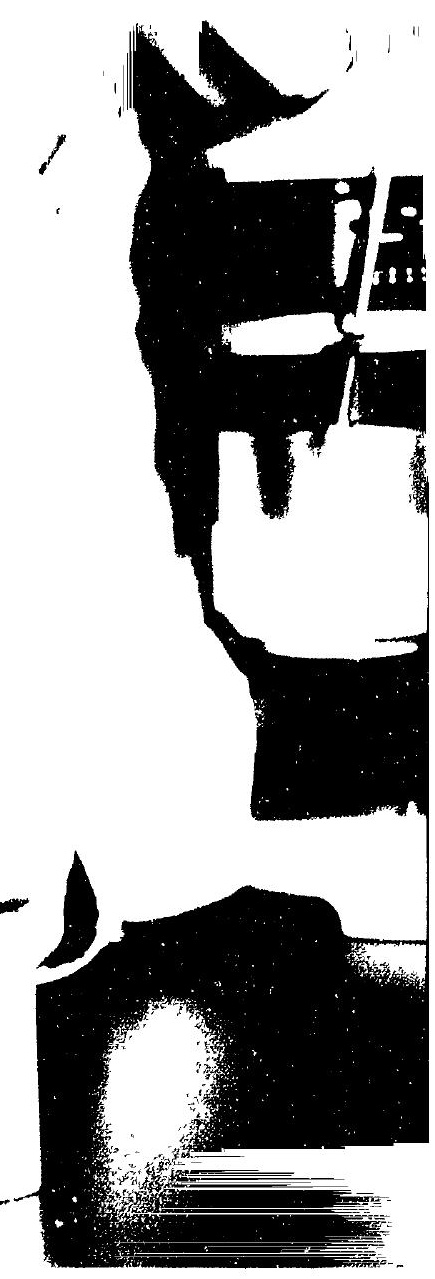


industrial furnaces, says Henry Street, of Sandia's Exploratory Batteries Department.

Other improvements in batteries have included the use of molybdenum as the positive terminal to further inhibit corrosion within the battery; the placement of a nickel grid in the lithium anode to increase efficiency; and the development of improved manufacturing techniques for eliminating cracking in the steel cell case.

Battery research is supported by DOE as a means of developing new technologies for meeting the nation's energy needs. Sandia's battery research is one of the earliest examples of transferring technology from a national laboratory to private industry, an activity that became a formal mission of the national laboratories with the National Competitiveness Technology Transfer Act, passed by Congress in 1989.

\section{Two kinds of lithium batteries}

Two kinds of lithium batteries now on the market use the glass headers developed by Sandia - lithium sulfur dioxide and lithium thionyl chloride. The more common of the two is lithium sulfur dioxide, which comes in 3-volt cells and has seven times the energy density of a typical alkaline battery. Lithium thionyl chloride has twice the energy density of lithium sulfur dioxide, meaning it lasts twice as long. It comes in cells of $31 / 2$ volts, making it possible to use fewer cells for the same application.

Sandia is using the lithium thionyl chloride batteries for nuclear surety applications, which require long-life power sources for command and control systems. Future uses will include stockpile modification and improvement plans. The British gas industry is also interested in this battery technology for use with redesigned gas meters. The British system will allow technicians to take meter readings of gas usage by telemetry without getting out of their vehicles. Britain is teaming with an Israeli company that will build the thionyl chloride cells to power the system.

The primary advantage of the Sandia-designed glass-to-metal seals is that they solved a recurring problem of glass corrosion in the battery industry, which in the past hampered efforts to develop a battery that would last beyond about 18 months.

"Glass electrically isolates the pin," says Street. "That's what allows you to have a battery with both positive and negative poles. If the pin were not isolated or the glass insulator were corroded, the battery would short-circuit."

Although manufacturers of consumer products prefer to use the glass headers in lithium batteries that present few hazards for everyday use, Sandia's batteries contain gaseous sulfur dioxide as the cathode material. Because the gas is toxic and under pressure, the batteries are vented to prevent case rupture.

Commercial battery manufacturers do, however, produce gaseous lithium-sulfur dioxide batteries for military applications. These batteries typically have headers of CABAL-12 glass. The armed forces use the batteries because they are lightweight, have high energy output, and can operate in frigid temperatures.

"Nobody had a thorough understanding of long-life battery performance until Sandia studied the problem," notes Street. "The battery group is probably one of the front runners at Sandia when it comes to solving problems with industry and introducing manufacturers to our technologies." 团

For more information, call

Henry Street, Org. 252.3, (505) 844-5029. 


\section{Cross-well imaging}

\section{Sandia-designed tool may boost oil production by finding unrecovered petroleum in existing fields}

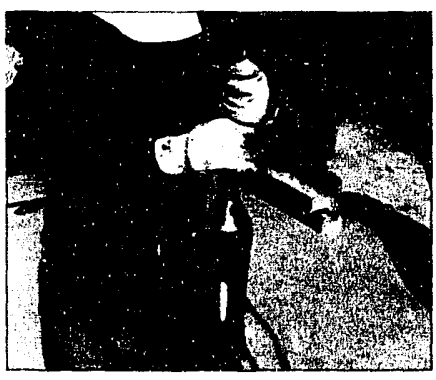

Sandia researcher Bruce Engler loads a seismic receiver into a well in La Habra, California. Bundled receivers allow several images to be formed at once.

ooking for
petroleum in been depleted, formations can go undetected inbetween existing petroleum-bearing wells. In the United States alone, an estimated 300 billion barrels remain undetected - equal to 60 to 100 years of normal production.

Typically, the oil industry uses seismic waves to generate images of what lies underground. Geologic strata alter seismic waves much the way a pane of glass muffles sound that passes through it. Using this principle, seismic waves transmitted from the surface can help identify oil-bearing formations. But the farther the seismic waves have to travel, the fuzzier the image.

Sandia and OYO Geospace Corp. have solved this problem by developing a new seismic detection system that generates much clearer images than surface seismic imaging - the method most commonly used by oil companies. Using a multistation borehole seismic receiver, the system is located much closer to the actual oil deposit and links several receivers together at different depths, all collecting signals simultaneously. Placed deep inside an existing well, the receivers detect seismic waves generated by an explosive or a vibrator placed in a neighboring well. This technique is known as cross-well imaging.

"If you're standing on the earth's surface and looking for an oil deposit that is 10,000 feet underground, the seismic energy you generate has to propagate down 10,000 feet and then back up to the surface," explains Sandia project leader Gerard Sleefe. "In cross-well imaging, the seismic energy only travels the distance between two wells, which may be 1,000 feet, and it travels only one way before it is detected."

Because of its closer location, the receiver detects the higher-frequency signals that would otherwise be lost over longer distances. The result is a more detailed image of rock formations and potential petroleum deposits. Because the system incorporates multiple devices, it saves time by forming several images at once.

"The alternative is to move the sensor around and run a new test at each depth," says Sandia engineer Ron Franco. "Each time we do a test using this new system, it's the equivalent of doing four or five tests using a single-level receiver."

The multilevel borehole array is only one example of many ongoing projects sponsored by the Department of Energy's Oil Recovery Technology Partnership with private industry. One of Sandia's primary missions is to support DOE in its efforts to meet national energy needs.

\section{Many potential applications}

"The multilevel borehole seismic receiver array represents a significant advance in technology," notes Sleefe. "For one thing, it monitors a broader

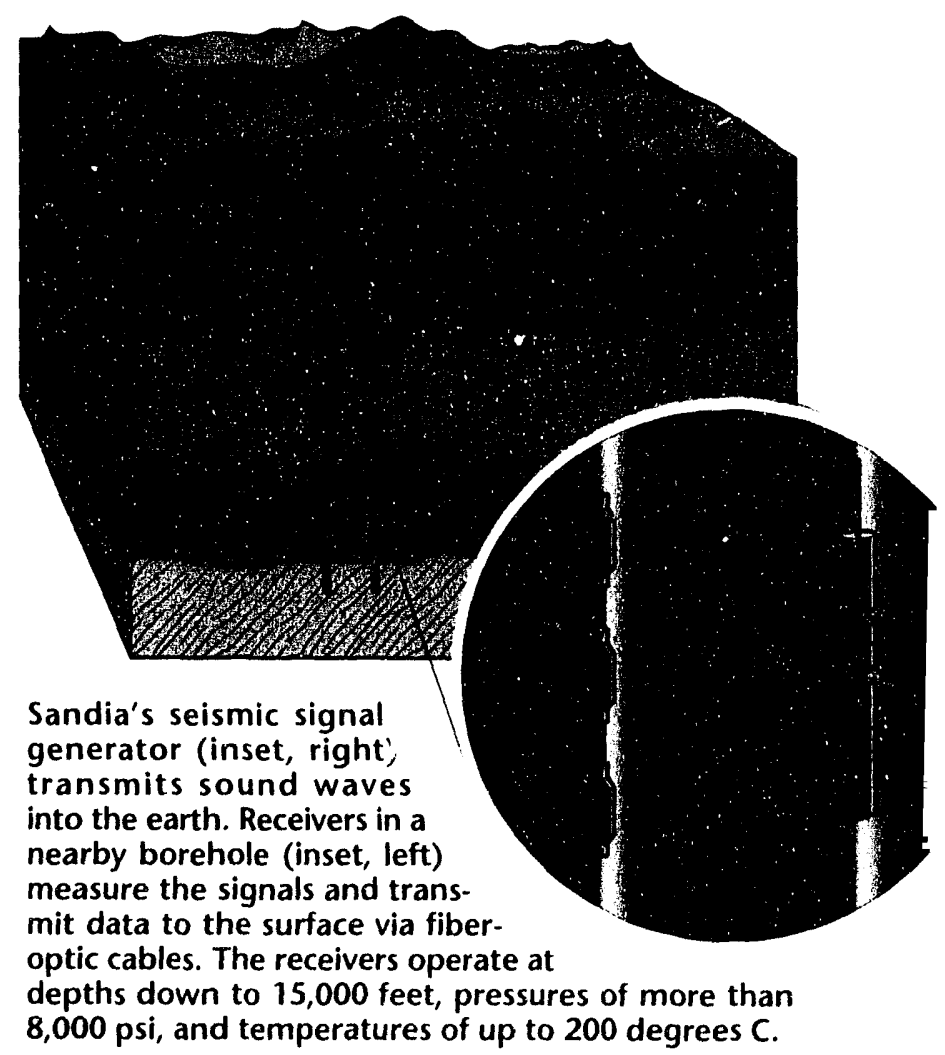


range of seismic frequencies. For another, it uses fiber-optic technology rather than conventional logging cable to transmit data to the surface. Fiber-optic cable can transmit a larger volume of data over a longer distance, which is significant when you're relaying information from three miles underground. Information arrives at the surface faster, allowing aboveground observers to get a more complete picture of what lies below."

The Sandia-OYO Geospace receiver is rugged and reliable even at the high temperatures and pressures encountered deep within the earth. The receiver clamps tightly to the borehole wall and does not resonate at frequencies that would interfere with seismic signals.

Motion detectors in the receiver-which are actually piezoelectric crystals that respond electrically to minute vibrations of the earth record the earth's response to the seismic-wave source. These detectors are much more sensitive to higher frequencies than a geophone, the device most commonly used in conventional receivers. The detectors measure the amount of energy absorbed by the earth and the time it takes for energy to travel from the source to the receiver. This information can be used to create an image of the earth's interior.

The technology has other potential applications as well. For example, it can be used to characterize underground waste deposits. Geologists can use the technique for mapping underground rock strata and groundwater. It can also be combined with tomographic imaging to create a three-dimensional view of the earth, much the way a neurosurgeon uses a CAT scan (computeraided tomography) to make an image of a brain tumor.

Last summer, Sandia and OYO Geospace engineers tested a new design that bundles five receivers into one unit, enabling seismic readings to be taken at five different depths at the same time. This significantly reduces the time needed

Researcher Jeff Morgan examines the inside of a borehole seismic receiver, part of a multistation system developed by Sandia for locating unrecovered petroleum in existing oil fields. Placed deep inside a well, the receivers detect seismic waves to produce images of what lies underground. to image an area, and in the oil industry, a little saved time saves a lot of money. Shutting down an active well to accommodate a cross-well survey can cost oil producers up to $\$ 50,000$ a day.

OYO Geospace has already placed a singlelevel version of the receiver on the market, and plans are under way to commercialize the fivelevel version as well. 田

For more information, call

Gerard E. Sleefe, Org. 6114, (505) 844-2195, or Ron Franco, Org. 2664, (505) 845-8654.

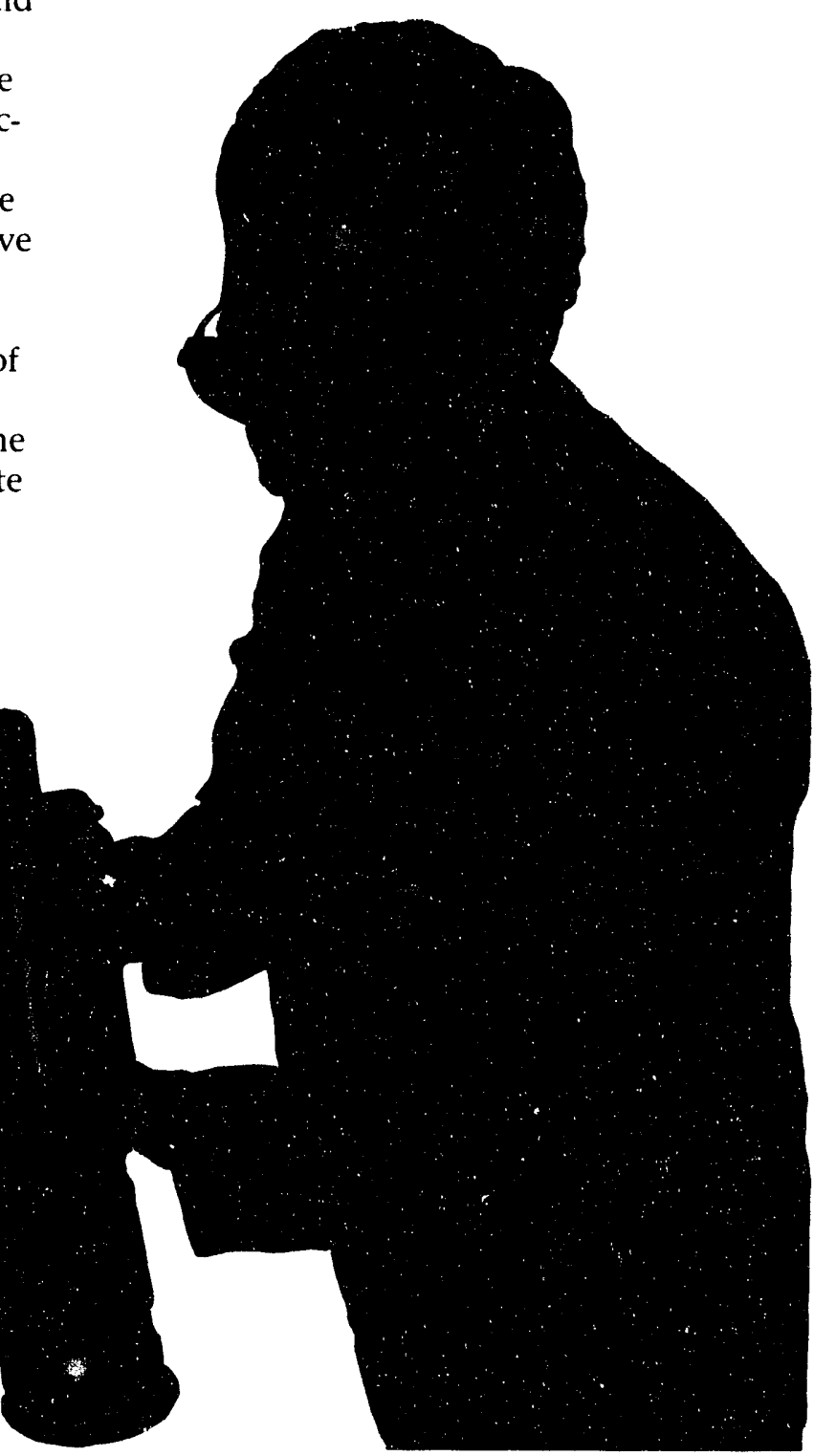




\section{Nuclear waste reduction}

\section{A new class of materials developed at Sandia selectively extracts cesium from radioactive waste}

B esides being pretty to look at - in snowflakes, cave formations, or gemstones - crystals have the useful quality of being structured in precise atomic layers with uniform spacing between them. These minute, atomic-scale spaces can capture certain molecules that fit between the layers the way a hand fits into a glove, while smaller molecules pass right through and larger molecules remain locked out.

This characteristic is being used at Sandia to separate cesium from radioactive waste, significantly reducing the volume of waste. A new class of materials known as crystalline silico-titanate ion-exchangers, developed by Sandia chemist Robert Dosch and chemical engineering professor Rayford Anthony of Texas A\&M, has tremendous potential for cleaning up nuclear waste.

Cesium-137 emits the most penetrating form of radiation - gamma rays. Even at low concentrations, it must be heavily shielded in order to be handled, making it a particular hazard for people who work with it. Cesium has a half-life

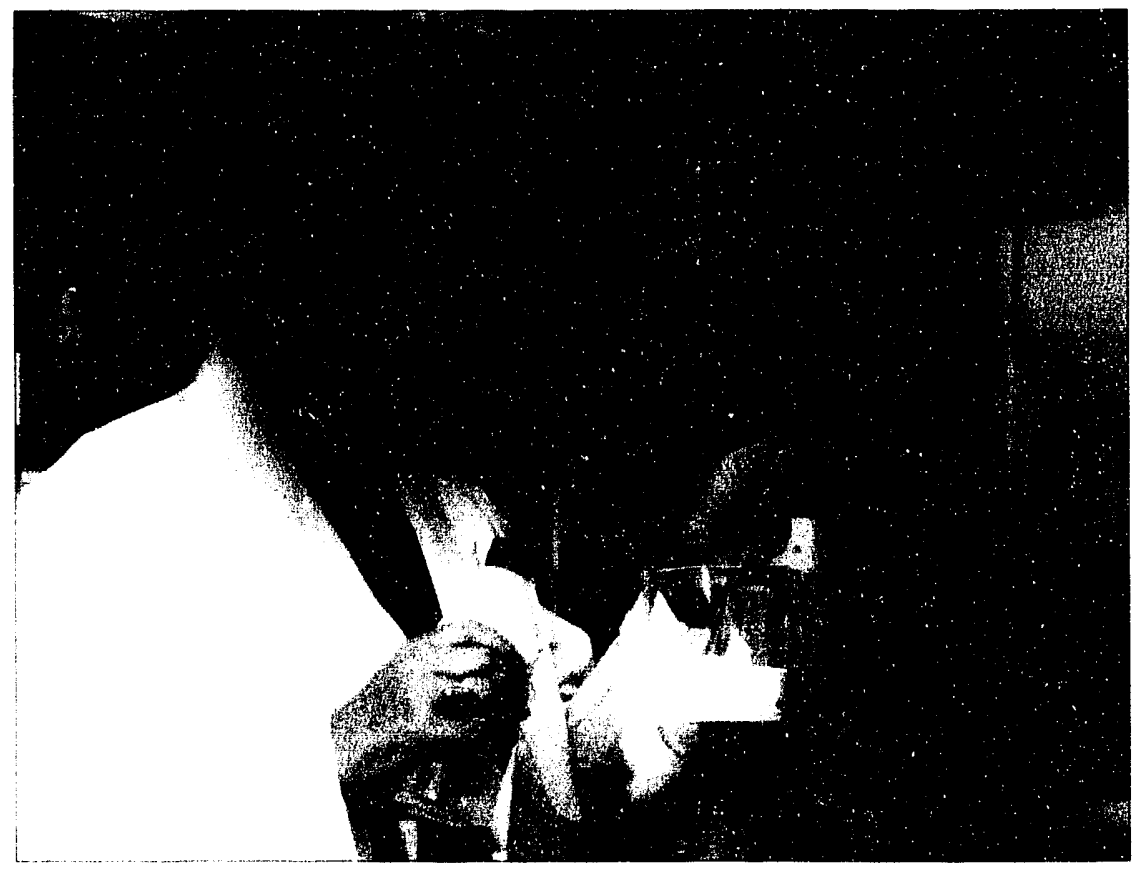

Chemist Linda McLaughlin does filtration of some titanate material such as that used for separating cesium from radioactive waste. of about 30 years, which means it takes 200 to 300 years to decay to safe levels.

Removing cesium makes the rest of the waste more acceptable to regulators and easier to process. The separated cesium then becomes part of a high-level radioactive waste stream that can be vitrified (hardened into glass) and shipped to a special repository such as the proposed Yucca Mountain site in Nevada.

\section{Grew out of work for DOE in 1970s}

The discovery of the silico-titanates grew out of work at Sandia in the 1970s for DOE on immobilizing high-level radioactive waste. The original amorphous titanate materials were used to isolate radioisotopes such as strontium-90 and plutonium to reduce the volume of the waste. These amorphous powders are now in large-scale use at DOE's Savannah River waste processing facility in South Carolina.

In 1981, Sandia researchers began to investigate the potential use of the materials as catalysts for converting coal to liquid fuel. They experimented with both amorphous powders and newer crystalline compounds, eventually discovering that the layered structure of crystalline compounds provided important catalytic and other properties. In 1992, they demonstrated that they could design crystalline silicotitanate structures that could sandwich cesium ions between layers or in the pores of crystalline material while leaving larger, hydrated sodium ions behind.

"It turns out that the crystalline compound is just a super material for removing cesium in the presence of high sodium concentrations, and that's what most of our defense wastes contain," says Dosch.

"It's kind of like a lock and key," adds Howard Stephens, manager of Sandia's Process Management Department. "Cesium goes into the lock, fits, and stays there; sodium 
doesn't. In fact, the ion-exchanging material is so sensitive to cesium in solution that it will remove one atom of cesium from 100,000 atoms of sodium."

The materials have great potential for processing radioactive wastes that contain small amounts of cesium-137 in high concentrations of sodium salts, such as those stored at the Hanford facility near Richland, Washington, and the Savannah River facility in South Carolina. Potential uses for the crystalline materials include remediation of contaminated soil and decontamination and decommission of nuclear facilities. Radioactive wastes stored in underground tanks at the Hanford facility typically contain sludge at the bottom, a water-soluble salt cake in the middle, and liquid waste on top. Cesium-137 is concentrated in the top two layers.

One potential method of treating the waste would involve adding water, along with both the new crystalline titanates and the older hydrous metal-oxide ion exchangers developed in the 1970s, to remove cesium and strontium, the primary sources of gamma radiation and heat inside the tanks. In another process, a water solution containing the waste is pumped over columns filled with the crystalline silicotitanates. The radioactive waste trapped by the titanate crystals could then be solidified into glass or ceramic form, and the remaining materials could be treated as low-level radioactive or chemical waste.

"This project appears to be getting a lot of attention worldwide," notes Larry Bustard, who serves as a Sandia liaison with the Hanford facility. "Inquiries have come in from Canada, Israel, Great Britain and others. A lot of people are interested in removing cesium and other elements from radioactive wastes. With DOE sponsorship, we are aggressively continuing to develop these materials for such applications."

For more information, call

Howard Stephens, Org. 6212, (505) 844-9178, or Larry Bustard, Org. 6624, (505) 845-8661.

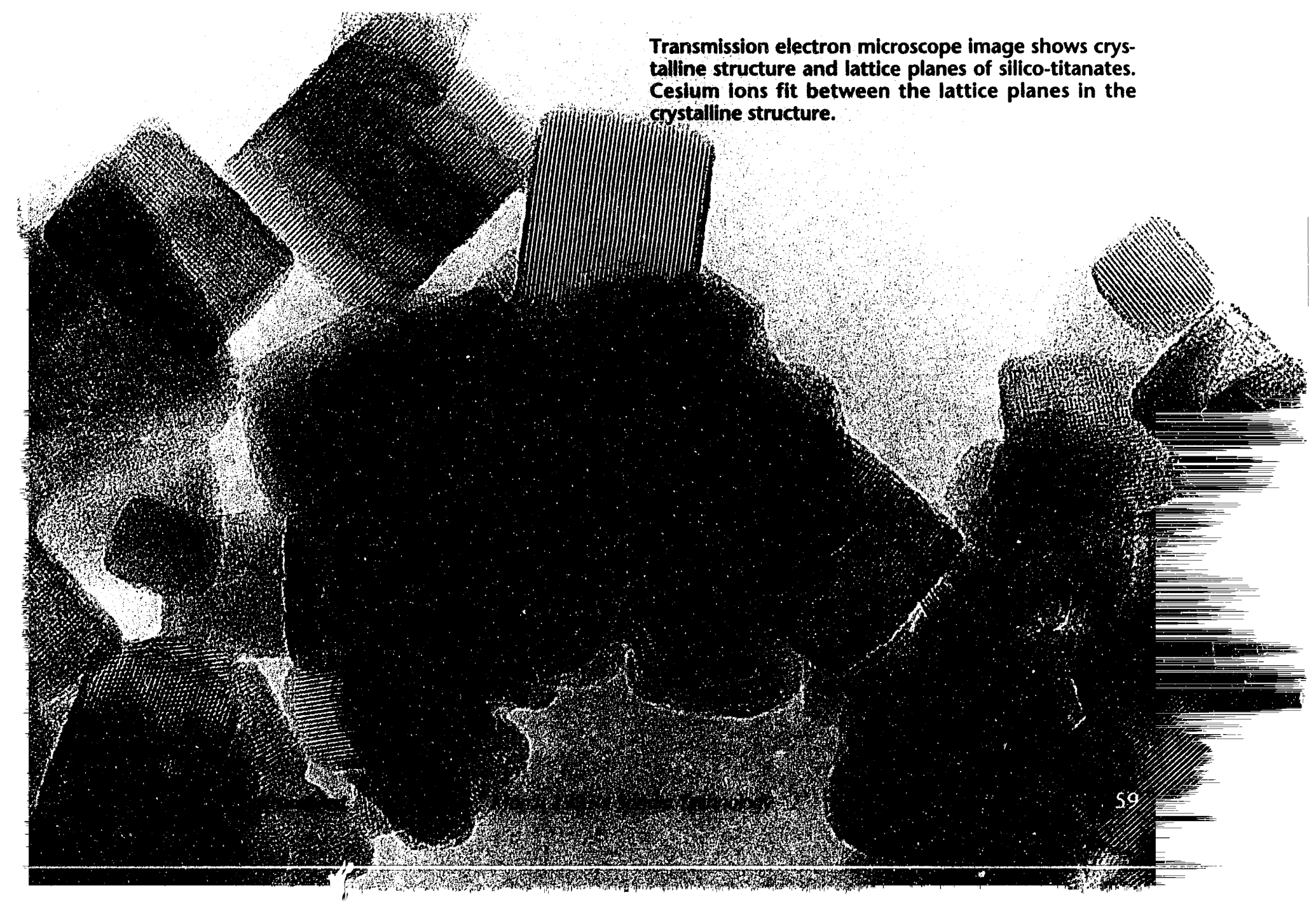




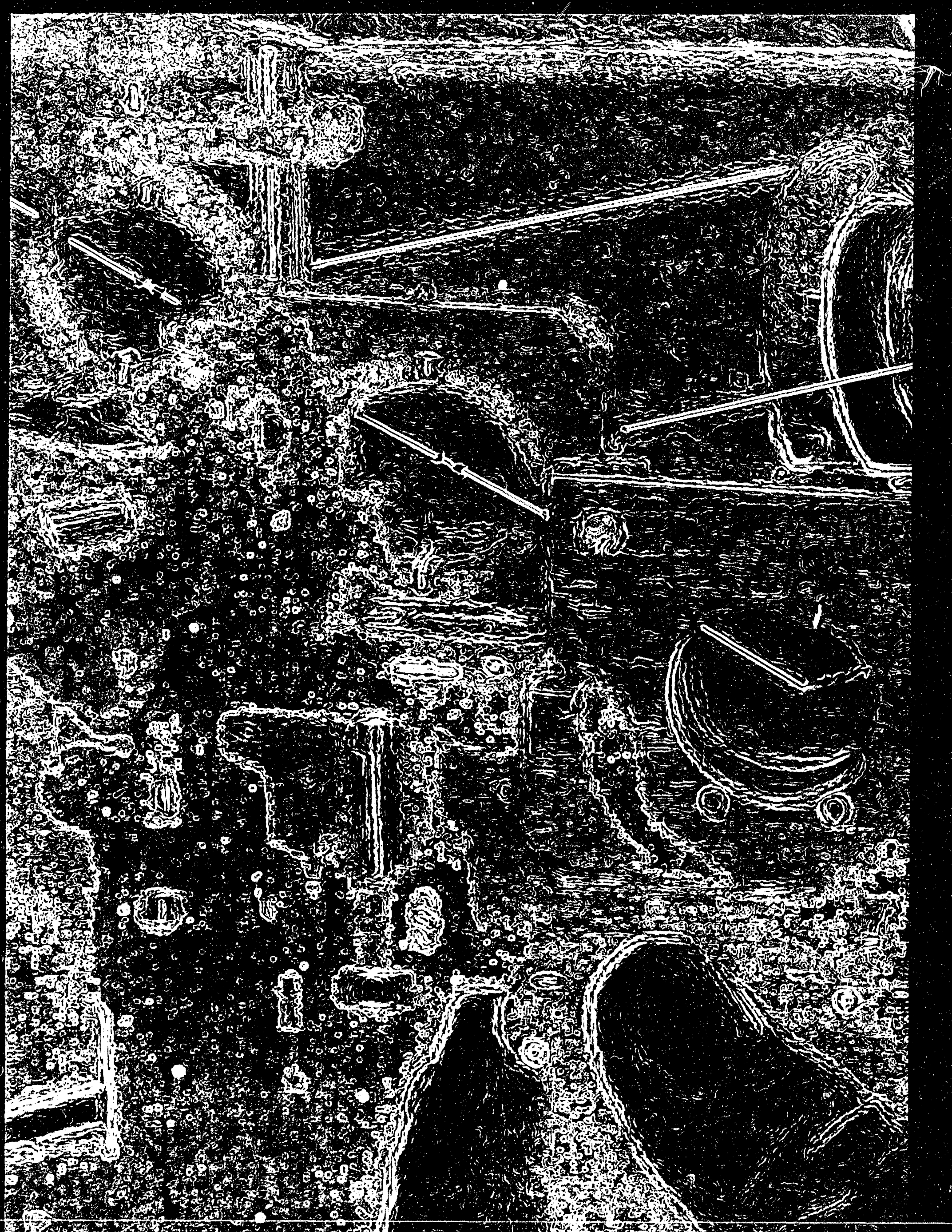




\section{Laser trigger}

\section{New inethod of detonation resists lightning, improves nuclear weapon safety assurance}

M aintaining the safety and security of the US nuclear weapon stockpile has always been of the utmost importance to scientists conducting research for DOE. Since researchers first began to formally consider the safety aspects of nuclear weapon design in the 1960s, three basic concepts have evolved for ensuring weapon safety-isolation of the warhead from threat environments, guaranteed inoperability of the weapon except during intentional use, and incompatibility of the explosive system with potential threat environments.

One of the more serious naturally occurring threats of concern to weapon designers is lightning. Conventional nuclear explosive systems contain detonators activated by electric currents. Such systems require robust shielding to protect them from large electric currents generated by lightning that could cause them to detonate.

Now, Sandia researchers, in a collaborative effort with Los Alamos National Laboratory,

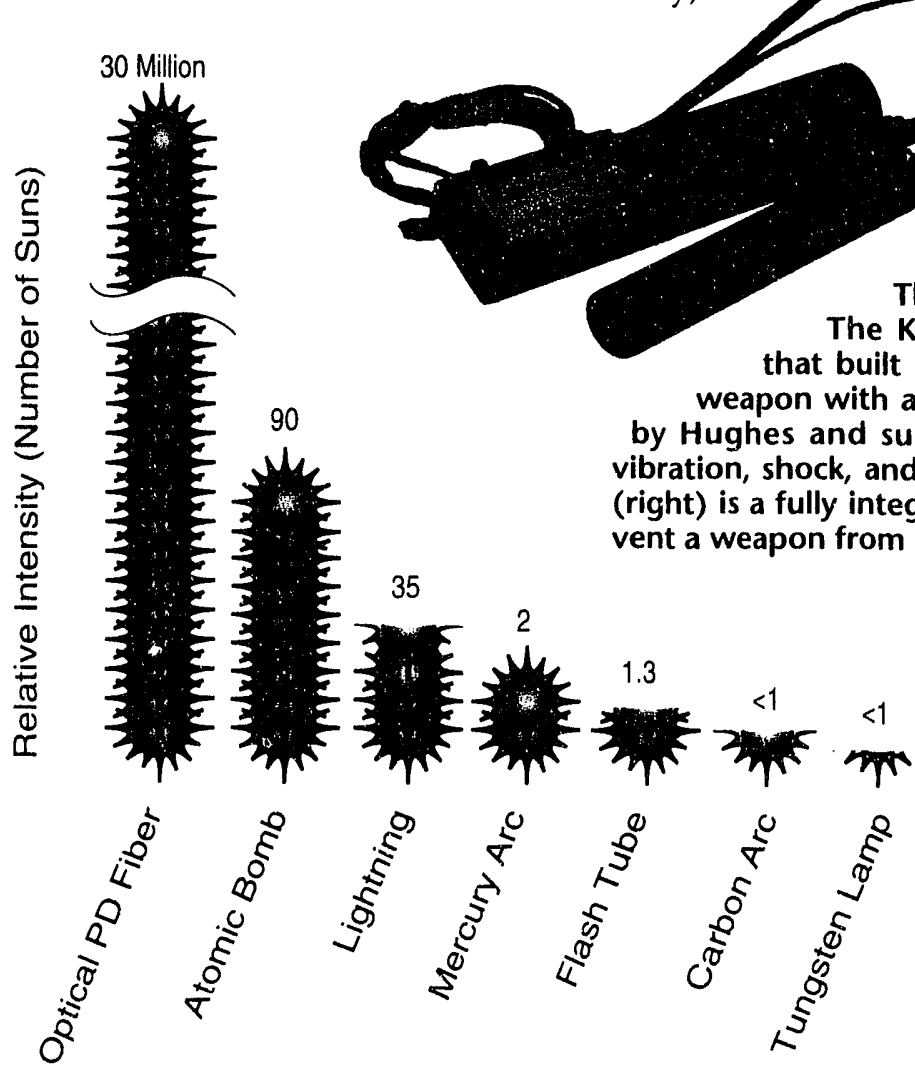

Allied Signal Kansas City Division, and Hughes Aircraft Company, have developed a new way of eliminating the threat of lightning vulnerability by replacing electrical energy in weapon detonation systems with laser light. Known as the Direct Optical Initiation (DOI) Project, the new approach does away with the need for shielding and makes the entire detonation system insensitive to lightning.

This work has spawned a variety of other improvements as well, including development of a very small laser that is capable of withstanding vibration, shock, and temperature extremes; integration of the laser into a complete firing system design; and characterization of the laserinduced optical damage thresholds of fiber-optic cables and other optical components.

These improvements have already been tested in simulated weapon environments and shown to be effective. "Some of the most exciting tests have been the demonstration of the fiber-optic system's ability to withstand lightning," says Kent Meeks, one of the developers of DOI.

Three generations of laser detonation:

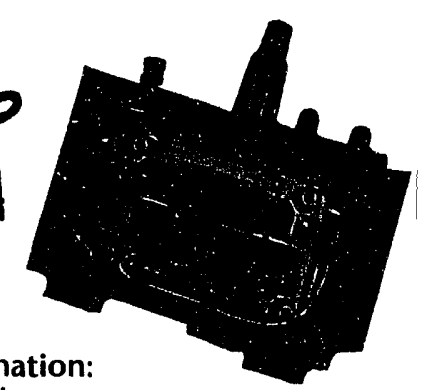

named for the company

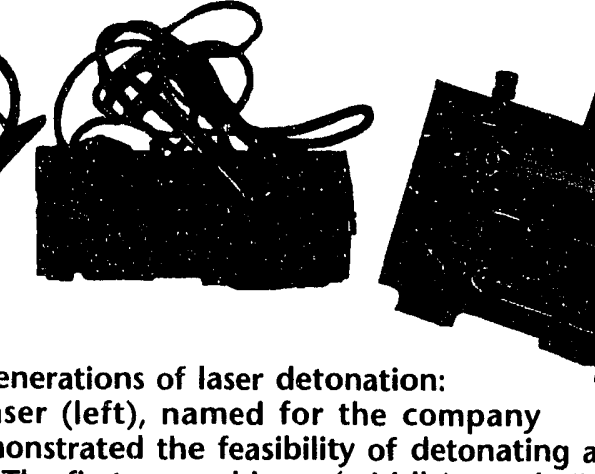
a 


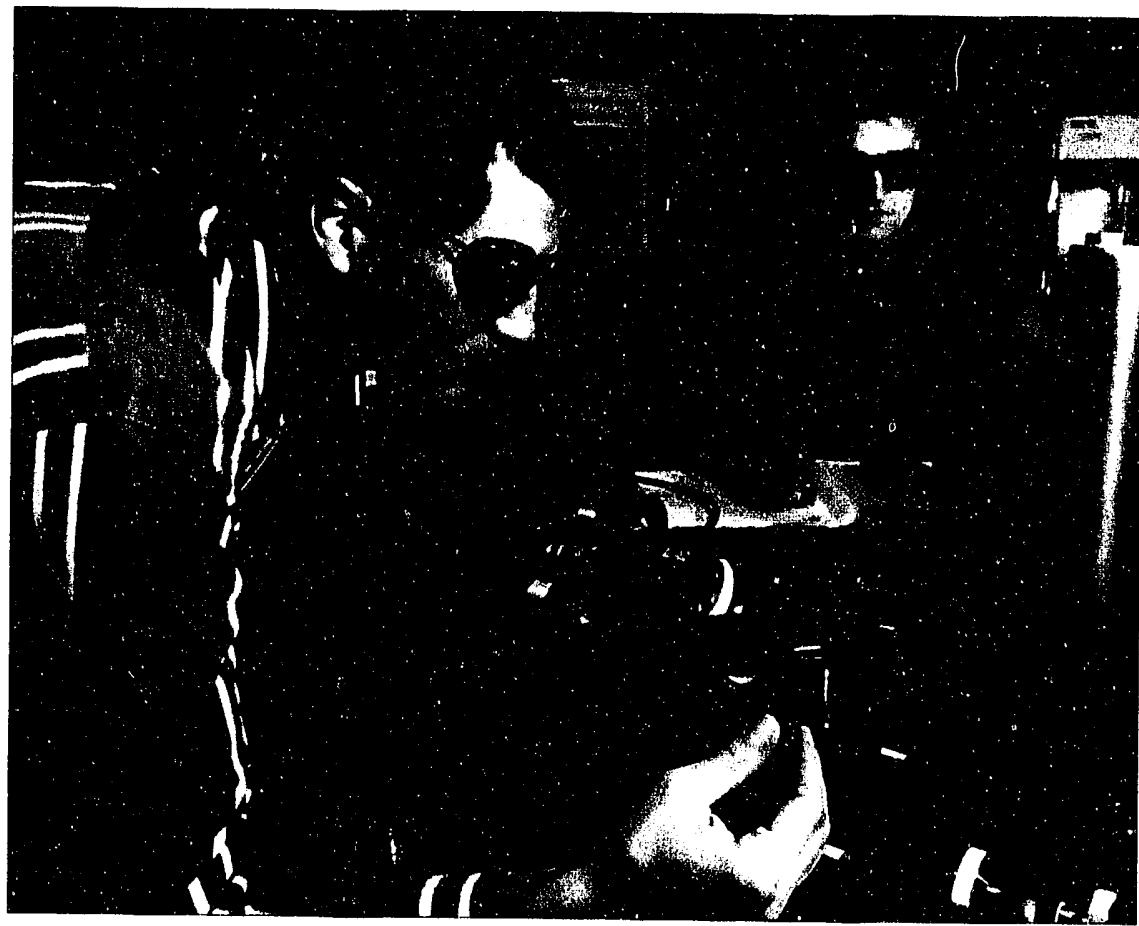

Their eyes shielded with protective goggles, researchers Dante Berry (left) and Jeff Alexander conduct an experiment with a laser designed to fire a detonator.

"During the past year, tests at Sandia's lightning simulator showed that the fiber-optic cables do not conduct lightning at currents of up to 200,000 amps. This is larger than 99 percent of all lightning strikes." However, even at 200,000 amps, the fiber-optic cables conduct less than 1 amp, an amount insufficient to set off even the most sensitive of nuclear weapon detonators.

\section{Only the laser can fire the detonator}

In fact, adds researcher Lou Weichman, the intensity of light required to fire the detonator system is much greater than that available from any source other than the specially designed laser used in the system. This means that the detonator is safe from naturally occurring hazards. Even the flash of another nuclear weapon would not provide enough brightness to fire the detonators, an operation that requires a brightness equal to 30 million suns. At luminosities below 300,000 suns, the coating at the ends of the fiberoptic cables acts as a mirror and simply reflects the light, leaving the detonator explosive unaffected. At luminosities between 300,000 and 3 million suns, the coating can melt but the amount of light is not sufficient to create the plasma needed to initiate the detonator.

The use of light has proved to be an effective means of achieving prompt detonation when intended. Developments during the past two years have taken this technology from concept to reality. In order to transport very high-energy densities from the laser to the detonators, researchers have improved detonation system designs to spread power uniformly over the fiber-optic cross section, thereby improving system reliability. They have also developed improved manufacturing and inspection techniques for building better fiber connections.

Laser-based firing systems could potentially reduce the need for underground nuclear testing. Existing nuclear safety designs require the firing set to be located close to the nuclear explosive in order to effectively shield both from potential lightning strikes. Since DOI does not require electrical shielding around the firing set to protect against lightning strikes, the firing set and nuclear explosive can be separated within the weapon without introducing nuclear safety vulnerabilities. This additional flexibility offers new opportunities for standardizing weapon design and reducing the need for nuclear testing of specific features.

For more information, call

Jack Jones, Org. 2505, (505) 844-8656. 


\section{STARS guidance and control}

\section{System guides target vehicles from Kauai to Kwajalein}

$S^{a}$ andia is developing a system to launch target vehicles and other payloads from the Kauai Test Facility in Hawaii to the Kwajalein area in the South Pacific. Called the Strategic Target System (STARS), the central element of this program is the STARS missile, a three-stage booster system designed specifically for flight testing in support of the Strategic Defense Initiative (SDI). The program sponsor is the Army's Space and Strategic Defense Command.

The navigation and guidance system for STARS, which includes a Sandia-designed flight computer and a Honeywell inertial measurement unit (IMU), has been the subject of several technology transfer requests from industry. The missile has also effectively utilized retired first- and secondstage rocket motors from the Navy's Polaris A3 fleet ballistic missile and combined them with new components such as a third-stage motor. Other features include completely redesigned electronic systems, telemetry, ordnance, and power systems.

The Kwajalein Missile Range is one of two US test ranges (White Sands, New Mexico, is the other) where testing of ballistic missile defense systems is allowed under international treaty. Although the distance from Kauai to Kwajelein is only a little more than 3,000 kilometers, the STARS system is designed to deliver targets with velocities typical of longer intercontinental ballistic missile trajectories. Two configurations of the missile, known as STARS I and II, have been developed for primary payload experiments. The STARS II configuration includes a fourth stage for additional maneuvering capability.

The STARS program depends on the support and expertise of the national test ranges; the aerospace companies that originally developed the first-, second-, and third-stage motors; and other industrial partners. Sandia's capabilities have been utilized in navigation, guidance, control, rocket systems, aerodynamics, structural analysis, telemetry, explosives, and environmental testing.

\section{Guidance and control technology}

Sandia has been active in the development of guidance and control technologies for maneuvering reentry vehicles for nearly 20 years. The design of the STARS guidance and control system is based on technologies previously developed for Department of Energy defense programs. STARS requires a rugged guidance and control system that is small in size and high in performance.

Booster systems are typically inertially guided. An inertial navigation system uses accelerometers to measure linear acceleration and integrates the results to obtain velocity and position. Accurate computation of the orientation of the accelerometers requires high-performance computers to handle complex calculations. To accomplish this, Sandia has integrated the algorithms for inertial navigation and guidance and flight control into a single flight computer known as the SANDAC V. The result has been greater efficiency and improved guidance and control.

The STARS I and II guidance systems use different inertial measurement units (IMUs). In STARS I, the IMU is a specially modified version of an Army land navigation system made by Honeywell that communicates with an external SANDAC V computer. The STARS II IMU was developed especially for Sandia by Honeywell.

The accuracy of an inertial guidance system depends on the ability of the sensors to measure accelerations and angular rotations of the vehicle as well as the quality of the models of the earth's shape and gravitational field and the initial alignment of the system. STARS guidance systems are capable of accuracies in Kauai-to-Kwajalein flights of 300 meters in the down-range direction and 1,500 meters in the cross-range direction.

To allow for even more precise navigation, Sandia is developing a means of allowing the missile's inertial navigation system to use measurements from the Global Positioning System (GPS) - a constellation of satellites in orbits 20,000 kilometers above the larth. Each satellite contains an atomic clock and transmits a message that includes time, position, and velocity of the satellite with respect to the Earth. The missile uses 
data from four or more of these satellites to estimate its position and velocity within 5 meters and 0.1 meter per second.

GIS receivers are readily available for use with aircraft or land vehicles. However, satellites and space systems travel at much higher velocities, making acquisition and tracking more difficult. One solution to this problem is to couple a GPS receiver with an inertial navigation system. The GPS measurements are then used to estimate the navigation errors of the inertial system. Data from the inertial system aids the receiver in acquiring and tracking satellites. The STARS system includes a six-channel GPS receiver designed by Texas Instruments.

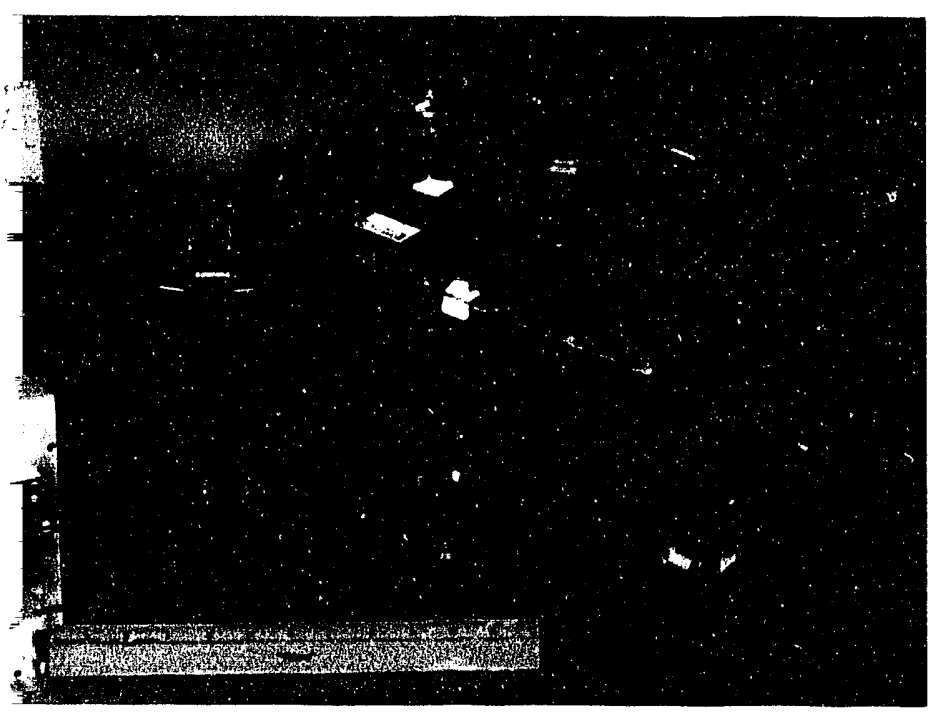

Although the STARS guidance and control system was developed specifically for SDI research, it is well-suited for guiding other space vehicles or aircraft. It could also be used with land vehicles if augmented by GPS measurements. Ultimately, the system will be useful for any application requiring precise navigation in a highly dynamic environment. 困

For more information, call

Al Watts, Org. 9101, (505) 844-6866.

The STARS guidance and control system comprises several major components, including (from left) the SANDAC V flight computer, a GPS receiver (front), a vehicle input-output package (back), the STARS II inertial measurement unit (IMU), and the STARS I IMU.

STARS guidance systems are used in a three-stage missile to deliver target vehicles and other payloads.

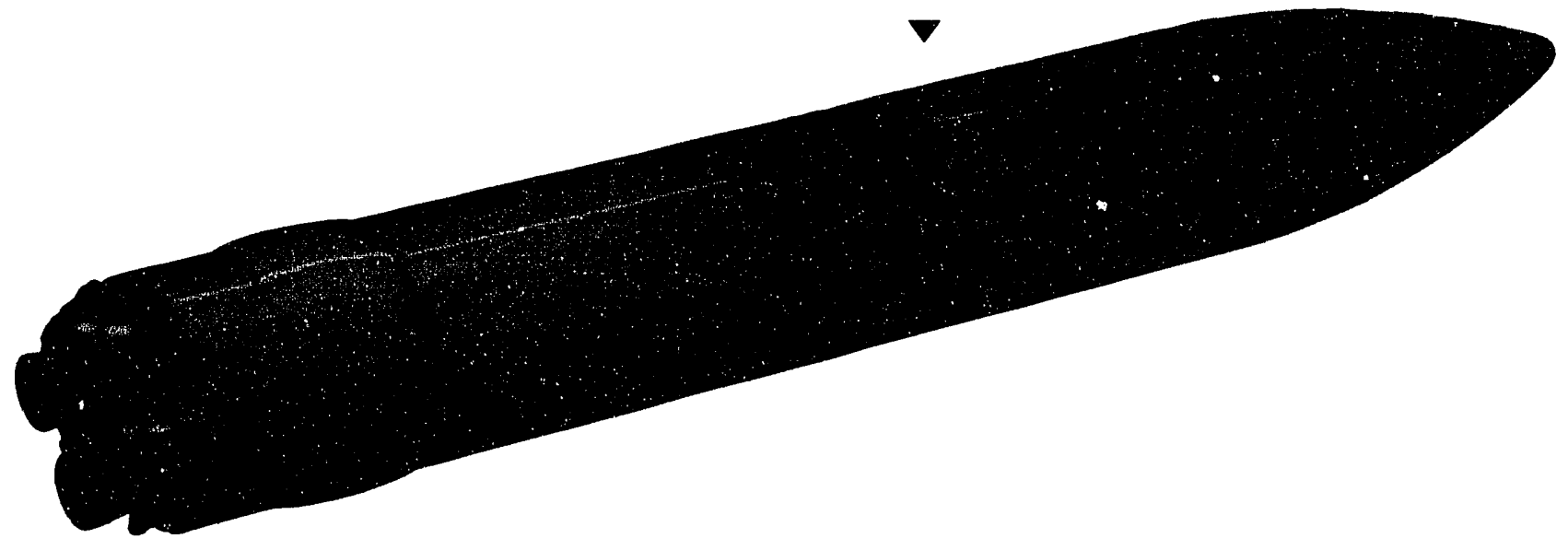





\section{Awards}

Professional society fellows \& members newly elected

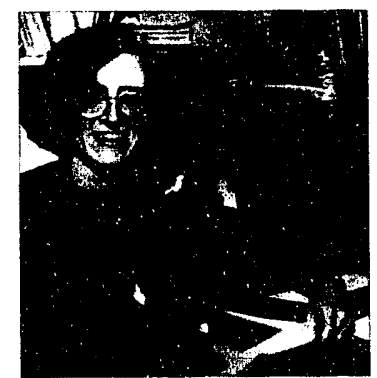

Mary Ann Sweeney
Gary Kelıogg

Sam Myers

Charles Hickox, Jr. Fred Norwood

Paul Peercy

Mary Ann Sweeney

Venkatesh

Narayanamurti

Del Owryoung

Don Sweeney

Nick Delollis

American Physical Society

American Society of Mechanical Engineers

Institute of Electrical and Electronics Engineers

National Academy of Engineering

Optical Society of America

Society for the Advancement of Material and Process Engineering

\section{Professional society awards}

Joe Michael

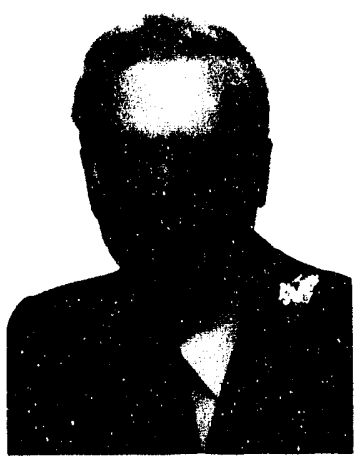

Paul Fleury

\section{Paul Fleury}

Bob Easterling

Mark Johnson

(U of C Florida)

Chris Nachtsheim

(U of Minnesota)

Tom Bement (LANL)

Steve Goods

Clarence Karfs

John Stormont
American Institute of Mining, Metallurgical, and Petroleum Engineers, Mechanical Working and Steel Processing Conference Meritorious Award for paper "Application of $\mathrm{x}$-ray microanalysis in the analytical electron microscope to materials research"

American Physical Society Frank Isakson Prize for contributions to the field of optical effects in solids

American Society of Quality Control Brumbaugh Award for paper judged to have the greatest contribution to the industrial applications of quality control

American Welding Society Warren F. Savage Award for paper published in Welding Joumal (5/92) - "Heliuminduced weld cracking in low heat input GMA weld overlays"

US National Committee for Rock Mechanics, PhD Thesis Award for thesis cited as a significant, original contribution to rock mechanics integrating laboratory testing, field measurements, and numerical studies

Federal Laboratory Consortium award for efforts to create a Microelectronics Quality/Reliability Center at Sandia

Research and Development Magazine R\&D 1(x) Award for developing a software package for modeling polymer alloys

Research and Development Magazine R\&I) 10() Award for a novel concept for helium self-pumping that could significantly reduce the size and complexity of the systems for vacuum pumping and tritium processing needed in future fusion reactors 


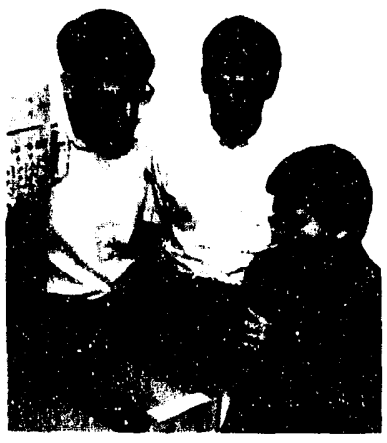

Lyndon Pierson, Tom Pratt, $\&$ Joseph Maestas
Lyndon Pierson

Joseph Maestas

Tom Pratt

Craig Tyner

Jim Pacheco

Mike Prairie

Larry Yellowhorse

John Holmes and NREL.

Peter Witze

Bill McCullough

Tom Priddy

\section{Department of Energy awards}

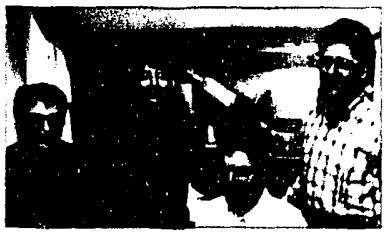

Alan Hurd, Carol Ashley, Randy Schunk, \& Jeff Brinker

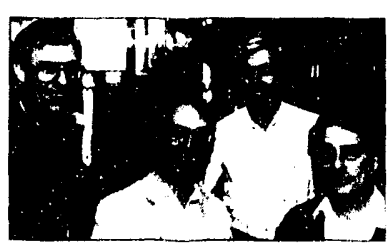

Jim Knapp, Barney Doyle, Tom Picraux, \& Sam Myers

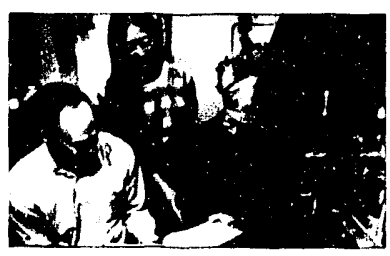

Mike Coltrin, Bill Breiland, Pauline Ho, Greg Evans, \& Bob Kee
Michael Mills

Daniel Miracle (Wright R\&D Center)

Jeff Brinker Alan Hurd Randy Schunk Carol Ashley

Barney Doyle Tom Picraux Jim Knapp Sam Myers Bill Breiland Mike Coltrin Greg Evans Pauline Ho Bob Kee

Pace VanDevender

Emil Kadlec Mark Kargel

\section{Department of Defense awards}

Jeffrey Everett

William Tedeschi

Research and Development Magazine R\&D 100 Award for development of improved hardware for recovery of synchronization loss in encrypted data Research and Development Magazine R\&D 100 Award for a system that uses sunlight to destroy organic toxins in ground water

Research and Development Magazine R\&D 100 Award for an automotive engine diagnostic tool that incorporates ionization probes in a head gasket for combustion analysis

Texas Tech University's Mechanical Engineering Academy charter members

DOE Basic Energy Sciences Materials Science Award for "High resolution transmission electron microscopy observation and embedded atom method calculation of dislocation cores in $\mathrm{NiAl}_{3}$ " DOE Basic Energy Sciences Materials Science Award for "Sol-gel film forrriation model"

DOE Basic Energy Sciences Materials Science Award for "Advanced ion beam techniques for materials analysis"

DOE Basic Energy Sciences Materials Science Award for "Chemical vapor deposition sciences"

DOE E. O. Lawrence Memorial Award for outstanding contributions to the generation of pulsed power

DOE/AL Quality Improvement Award for contributions to improved quality and attendant cost reductions through the design and development of the Built-in-Self Test System for the MC4130 telemetry package for the B61

Office of the Secretary of Defense Medal for Outstanding Public Service

Defense Nuclear Agency Director's Award for Meritorious Public Service 
Acoustic data transmission through a drillstring Patent \#5,128,901

Douglas S. Drumheller

Acoustic wave device using plate modes with surface-parallel displacement

Patent \#5,117,146

Stephen J. Martin, Antonio J. Ricco

Apparatus for and method of operating a cylindrical pulse induction mass launcher P'atent \#5,125,321

Maynard Cowan, Jr., Billy W. Duggin,

Melvin M. Widner

Backscattering spectrometry device for identifying unknown elements present in a workplace

Patent \#5,059,785

Barney L. Doyle, James A. Knapp

Beacon data acquisition and display system Patent \#5,073,779

David G. Skogmo, Billy D. Black

Carrier-lifetime-controlled selective etching process for semiconductors using electron launching voltage

Patent \#5,092,957

Carol I. H. Ashby, David R. Myers

Composition containing aerogel substrate loaded with tritium

Patent \#5,078,919

Carol S. Ashley, C. Jeffrey Brinker,

Robert E. Ellefson, John T. Gill,

Scott Reed, Robert J. Walko

Direct write with microelectronic circuit fabrication

Patent \#5,132,248

Timothy Drummond, David Ginley

Downhole hydraulic seismic generator

Patent $\$ 5,113,966$

Danny L. Gregory, Harry C. Hardee,

David O. Smallwood

Dual output acoustic wave sensor for

molecular identification

Patent \#5,076,094

Gregory C. Frye, Stephen J. Martin

Feedback stabilization system for pulsed single longitudinal mode tunable lasers

Patent $\# 5,054,028$

Peter Esherick, Thomas D. Raymond

Fiber-optic liquid level sensor

Patent \#5,(072,617

Jonathan D. Weiss
Fiber-optic strain gauge with attached ends and unattached microbend sections

Patent \# 5,132,529

Jonathan D. Weiss

Hand-held explosives detections system

Patent $\# 5,138,889$

Frank J. Conrad

Hot filament CVD of boron nitride films

Patent \#5,079,038

Robert R. Rye

Hybrid sol-gel optical materials

Patent \#5,130,397

John M. Zeigler

Liquid metal electric pump

Patent \#5,080,559

Joseph P. Abbin, Charles E. Andraka, Laurance L.

Lukens, James B. Moreno

Long wavelength, high-gain InAsSb strained layer superlattice photo-conductive detectors Patent \#5,065,205

Robert M. Biefeld, L. Ralph Dawson,

Ian J. Fritze, Steven R. Kurtz,

Thomas E. Zipperian

Method and apparatus for the control of fluid dynamic mixing in pulse combustors Patent \#5,118,281

T. Tazwell Bramlette, Jay O. Keller

Method for simultaneous overlapped communications between neighboring processors in a multiple

Patent $\# 5,072,371$

Robert E. Benner, John L. Gustafson,

Gary R. Montry

Method for the preparation of metal colloids in inverse micelles and product preferred by the method

Patent \#5,147,841

Jess P. Wilcoxon

Method of making an ion implanted planarburied-heterostructure diode laser

Patent \#5,102,825

Thomas M. Brennan, Burrell E. Hammons, David R. Myers, Gregory A. Vawter

Molecular sieve sensors for selective detection at a nanogram level

Patent \#5,151,110

Thomas Bein, Kelly D. Brown,

Gregory C. Fyre, Charles J. Brinker 
Nonvolatile semiconductor memory having three dimension charge confinement Patent \# 5,055,890

l. Ralph Dawson, Gordon C. Osbourn, Paul S. Peercy, Harry 'T. Weaver,

Thomas E. Zipperian

Optical pattern recognition architecture implementing the mean-square error correlation

Patent \#5,060,282

Perry A. Molley

Photochemical etching monitor

Patent \#5,097,200

Clifford W. Mendel, Mark E. Savage

Precision wire feeder for small diameter wire Patent \#5, 137,223

Eldon D. Brandon, Frederick M. Hooper, Marvin I.. Reichenbach

Quartz crystal growth

Patent \#5,1.35,603

Richard J. Baughman

Rotary drive mechanism

Patent \# 5,055,727

Eugene $W$. Kenderdine

Sealing glasses for titanium and titanium alloys

Patent \#5, 104,7.38

Richard K. Brow, Randall D). Watkins
Solder extrusion pressure bonding process and bonded products produced thereby

Patent \#5, 121,87

I. C. Beavis, M. M. Karnowsky, I. G. Yost

Solid-state radiation-emitting compositions and devices

Patent \#5,122,305

Carol S. Ashley, C. Jeffrey Brinker,

Scott Reed, Timothy J. Shepodd,

L. E. Leonard, Robert E. Ellefson

John T. Gill, Robert J. Walko,

Clifford L. Renschler

Solid-state radiation-emitting compositions and devices

Patent \#5,137,659

Carol S. Ashley, C. Jeffrey Brinker,

Scott Reed, Robert J. Walko

Solid-state radioluminescent zeolitecontaining composition and light sources Patent \#5,100,587

Roger L. Clough, John T. Gill

Daniel B. Hawkins, Clifford Renschler, Timothy

J. Shepodd, Henry M. Smith

Triaxial thermopile array geo-heat-flow sensor Patent \#5, 121,993

Charles R. Carrigan, Harry C. Hardee, Gerald D.

Reynolds, Terry D. Steinfort

Selective protection of poly (tetra-

fluorethylene) from effects of chemical

etching

Patent \#5,066,565

Robert J. Martinez,

Robert R. Rye

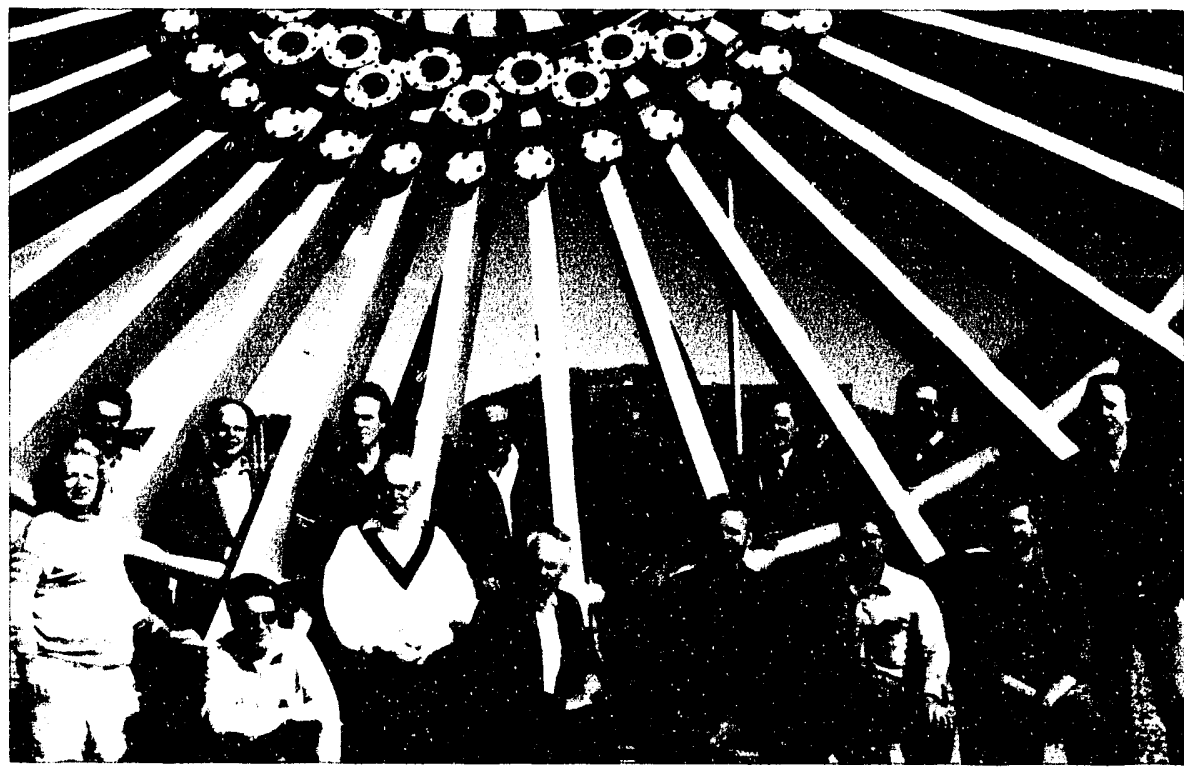




\title{
Corporate Publications, Sandia National Laboratories
}

\author{
Writing and Editing \\ Linda Doran (lead) \\ H. L. Floyd \\ Bob Goetsch \\ Jim Leonard \\ Lori Parrott \\ John Stikar (TRI)
}

Graphic Design and Illustration

Jim Bolton (TRI)

Toby Dickey

Carl Hamberg (TRI)

Janet Jenkins

Kay Rivers-Stroup

Photography

Jim Bechdel

Walt Dickenman

Lynda Hadley

Randy Montoya

Mark Poulsen

AT\&T

U.S. Air Force

Publications Coordination

Debbie Johnson

(505) 844-4902

\section{Sandia National Laboratories}

SAND92-2395

DOE Distribution Category UC900
Send change of address (include old address) and requests for additional copies to the address below, or telefax to (505) 844-1392.

Sandia National Laboratories Corporate Publications Dept. 4526

P.O. Box 5800

Albuquerque, NM 87185-5800
This report was prepared as an account of work sponsored by the United States Government. Neither the United States nor the United States Department of Energy, nor any of their employees, nor any of their contractors, subcontractors, or their employees: (1) make any warranty, express or implied, or assume any legal liability or responsibility for the accuracy, completeness or usefulness of any information, apparatus, product or process disclosed, or represent that its use would not infringe privately owned rights; or (2) endorse or approve either expressly or by implication any apparatus, product or process or the use thereof covered by this report.

\section{Printed in the United States of America}

Available from National Technical Information Service

U. S. Department of Commerce

5285 Port Royal Road

Springfield, Virginia 22161

(703) $487-4600$

NTIS price codes

Printed copy: A04

Microfiche copy: A01 

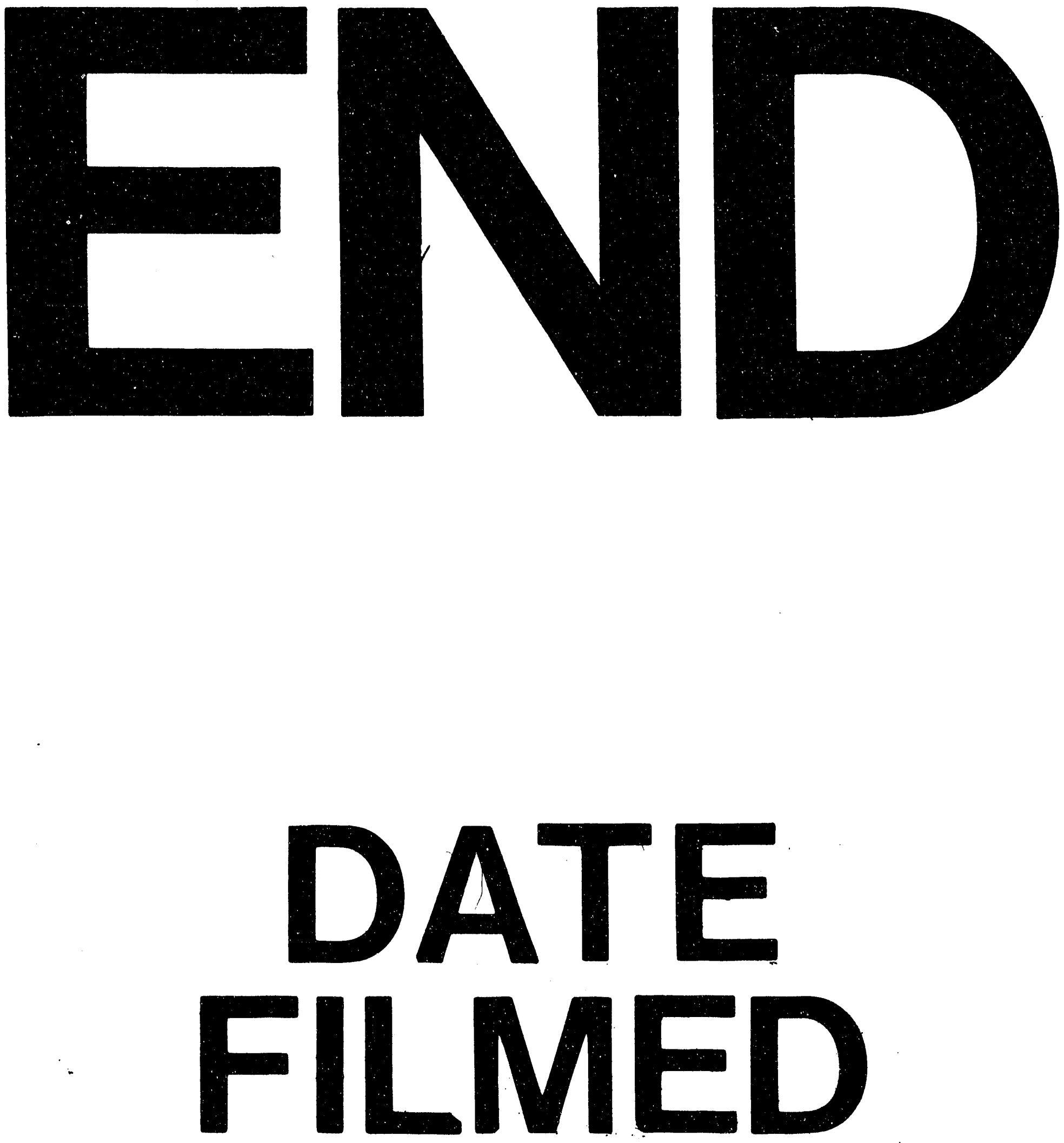

I

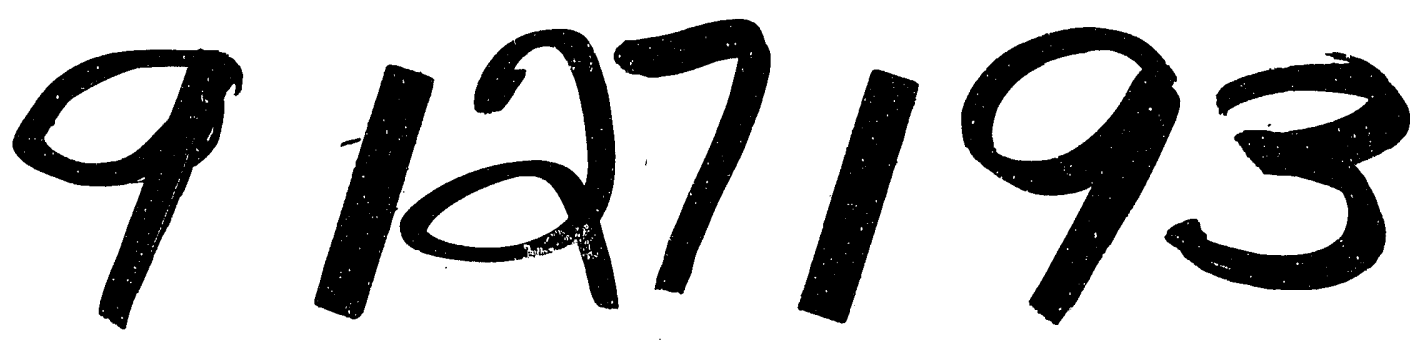


